\title{
Creating 3D Human Character Mesh Prototypes from a Single Front-View Sketch
}

\author{
by
}

Shaikah Bakerman

A thesis submitted to the Faculty of Graduate and Postdoctoral

Affairs in partial fulfillment of the requirements

for the degree of

Masters of Computer Science

in

Human Computer Interaction

Carleton University
Ottawa, Ontario

(C) 2016

Shaikah Bakerman 


\section{Abstract}

3D character modeling, a vital part in film and video game production, is a process that starts by blocking out the basic geometry of a character, which is then transformed into a detailed and enhanced mesh. This process can be a long and daunting task to novice modelers. As a result, extensive research in the area of sketch-based modeling has focused on finding solutions that facilitate this process.

We developed a sketch-based modeling system that constructs the basic $3 \mathrm{D}$ geometry of a human character mesh based on a single front-view sketch and minimal user interaction through a simple interface. The main objective of this system is to help novice modelers by automating the initial phase of the modeling process with the aid of an intuitive user interface, and to construct a basic mesh with suitable structure that conforms to common modeling techniques.

We conducted a user study to evaluate the system's user interface and produced mesh. Results show that our system's user interface is intuitive and easy to use. In addition, the system produces suitable meshes that modelers considered usable to facilitate the modeling process. Moreover, the processing time of our system is faster than the average time it takes modelers to manually construct a similar mesh, an advantage that benefits modelers who value the speed of a sketch-based modeling system, in addition to the accuracy of its results. 


\section{Acknowledgments}

I would like to give special thanks to my supervisor, Professor Chris Joslin, for sharing his valuable knowledge, for his guidance and support, and his patience throughout this journey.

I would also like to thank Ms. Erenia Hernández Oliver, the graduate administrator at the School of Information Technology, for her unconditional support and for always willing to give the advise and help I needed. I'm also thankful to all the staff at the School of Information Technology, who helped me during user study recruitment and set up. Their effort is deeply appreciated.

I would like to thank my HCI friends, especially Rufino Ansara, with whom I studied, worked, took coffee breaks and enjoyed every step of the journey.

I would like to thank Eman Karakotly and Faisal Rasim for drawing and preparing the lead character sketch used in this thesis to illustrate my research methodology. Their astonishing creativity and talent have always inspired me.

In addition, I want to thank everyone who contributed with their sketch and shared their art to demonstrate my results in this thesis.

Finally, I would like to express my deepest love and appreciation for my family: my parents, my sister Shahad, my brother Abdullah and my baby cat Sarabi. Your unconditional love and support are my constant motivation. Thank you for staying up late with me to give me a boost and happily make me coffee and snacks while I worked on my thesis. Thank you for your encouraging words, and for the warm hugs when I most needed them, and thank you for believing in me more than I believed in myself. I could not have done this without you. I love you guys very much. This is for you. 


\section{Table of Content}

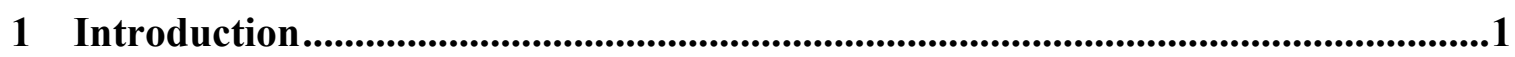

1.1 Sketch-Based Modeling ...................................................................................

1.2 Problem Description ................................................................................ 4

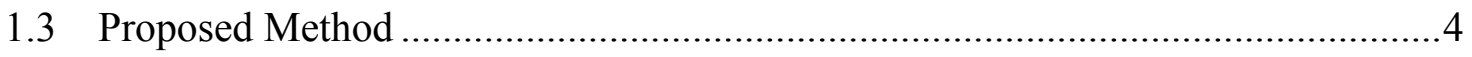

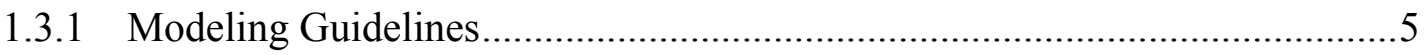

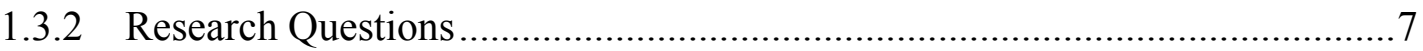

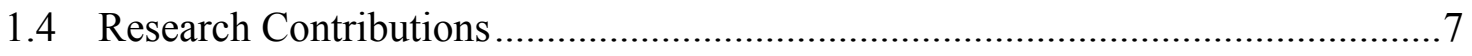

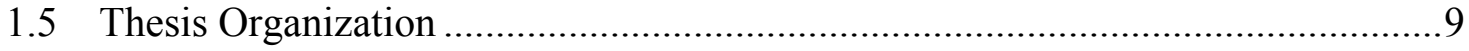

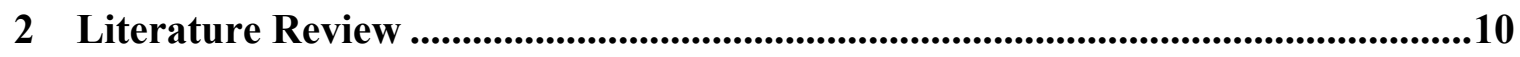

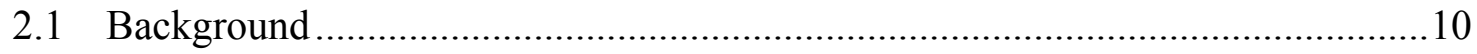

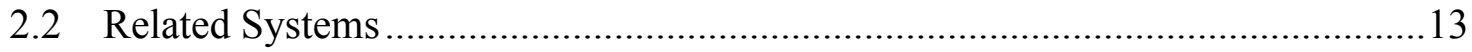

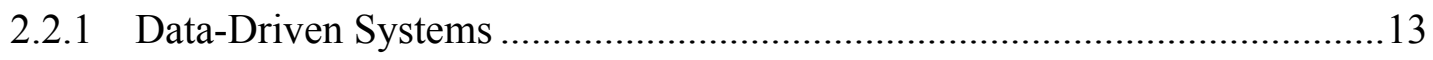

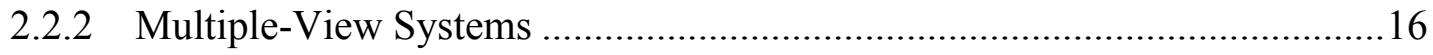

2.2.3 Single-View, No-Database Systems .................................................. 17

2.2.3.1 Fully Automatic Construction From Concept Art ............................... 17

2.2.3.2 3D Skeleton-Based Construction of Cartoon Drawings ...........................19

3 Methodology .........................................................................................................................22

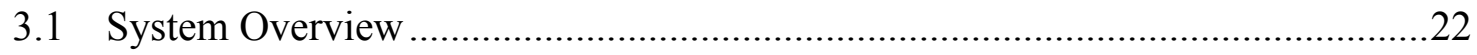

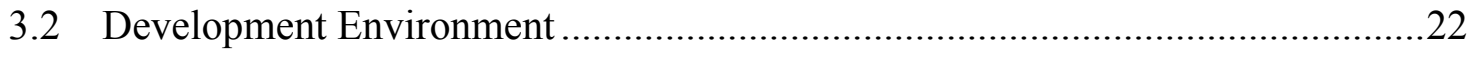

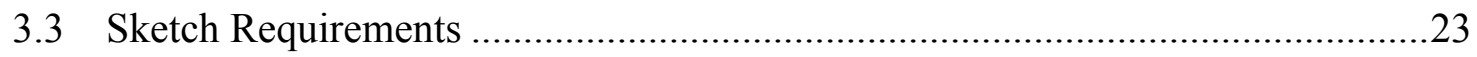

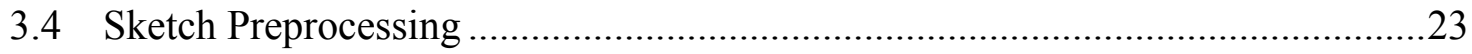




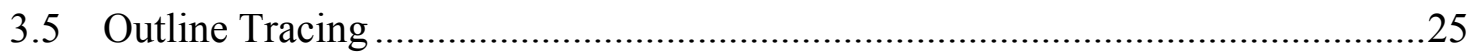

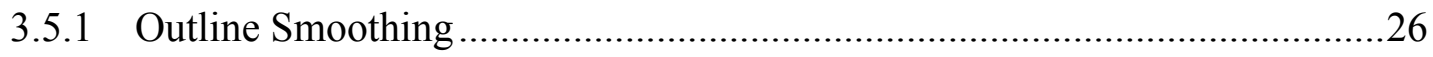

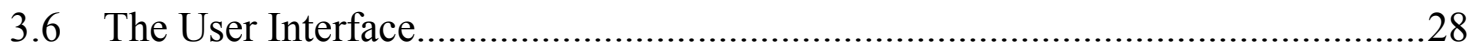

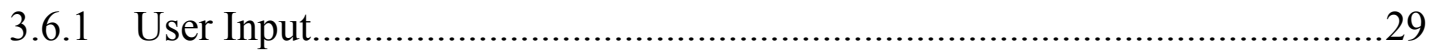

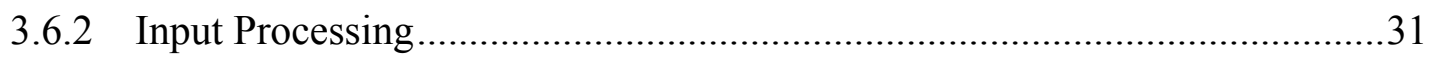

3.7 2D Character Processing ..................................................................................32

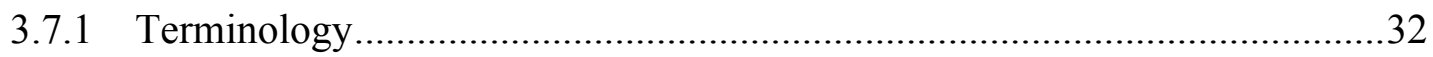

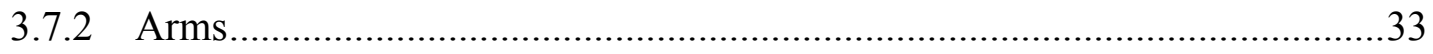

3.7.2.1 The Intersection Algorithm.....................................................................34

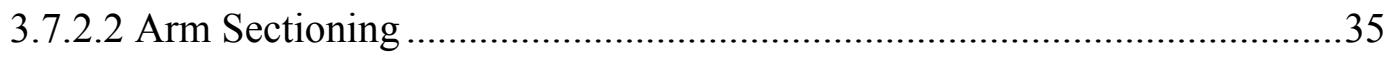

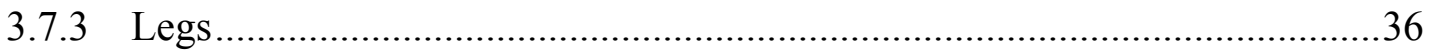

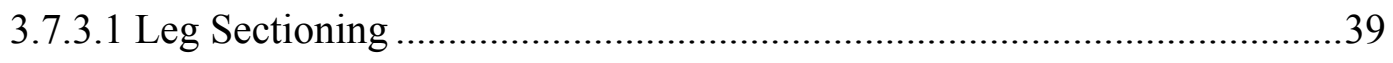

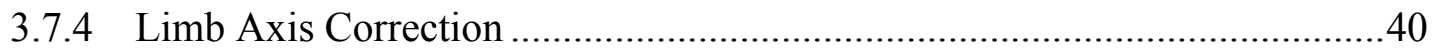

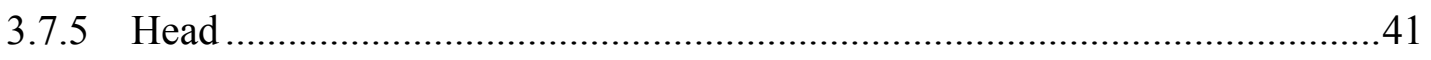

3.7.5.1 Neck Sectioning ...............................................................................4

3.7.5.2 Estimating Head Dimensions..............................................................43

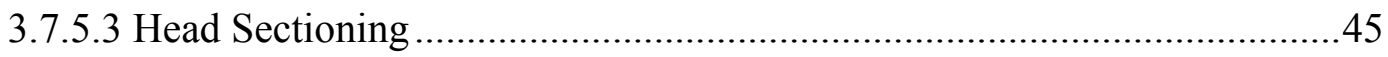

3.7.5.4 Neck Width Revisited ..........................................................................47

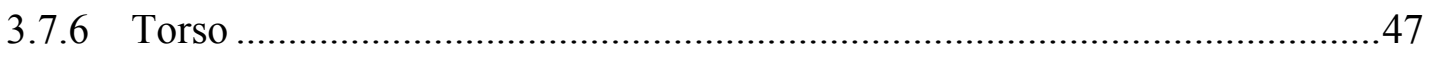

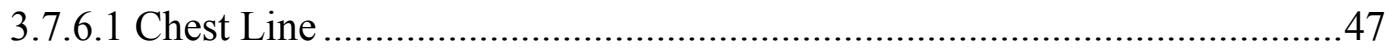

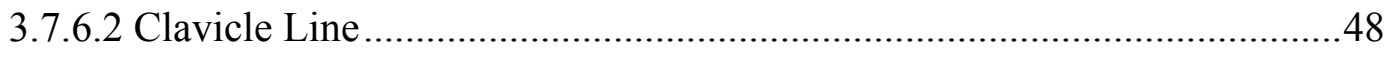

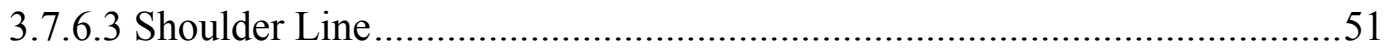

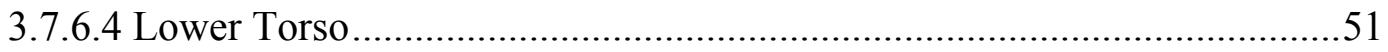




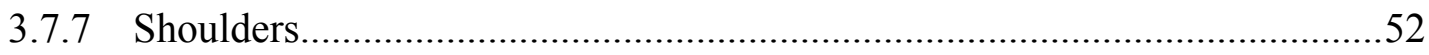

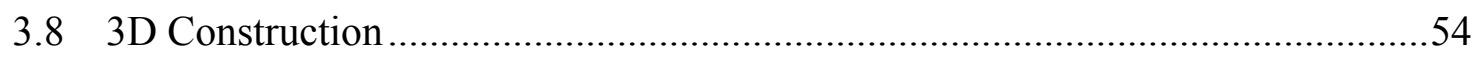

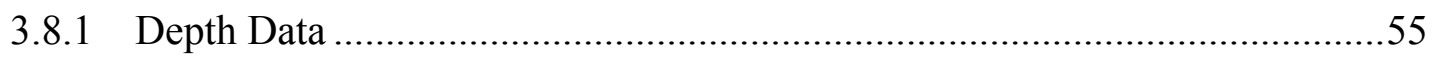

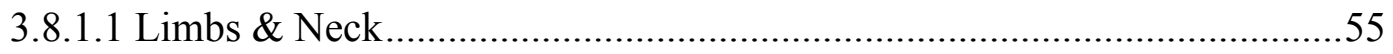

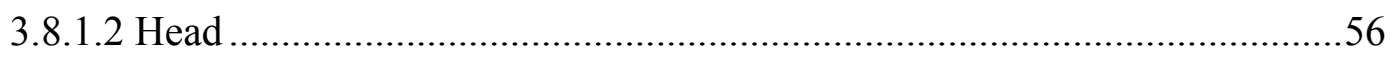

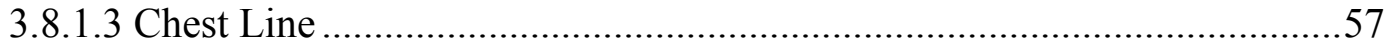

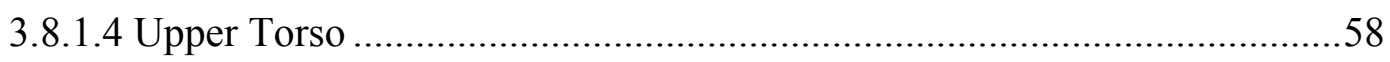

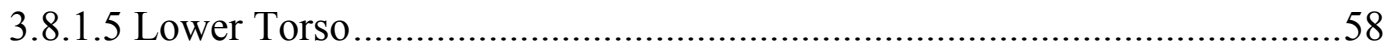

3.8.1.6 Torso Alignment ................................................................................59

3.8.2 Mesh Construction \& Smoothing …………………..................................60

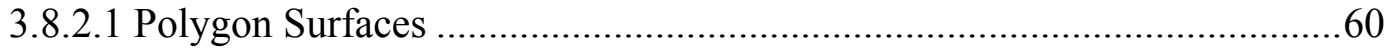

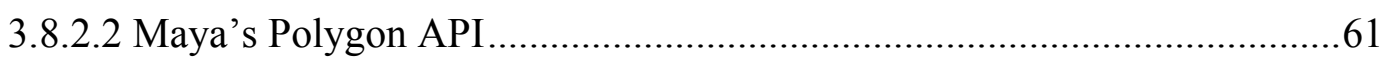

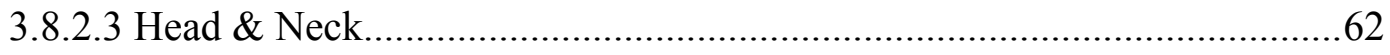

3.8.2.4 Torso Depth (Front Adjustment) ..................................................................66

3.8.2.5 Combining Torso \& Legs ...................................................................67

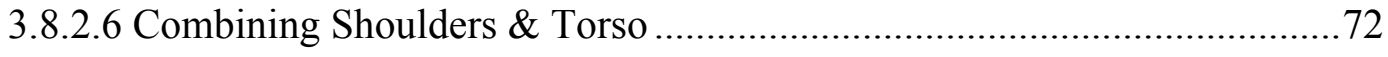

4 Experiments and Results................................................................................................76

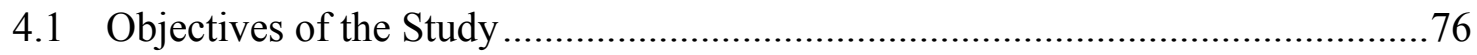

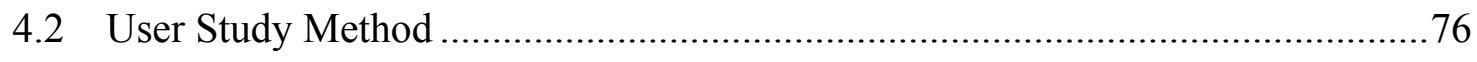

4.3 Hardware

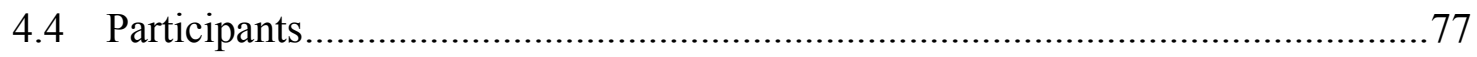

4.4.1 Levels of Experience ...............................................................................

4.4.2 Automatic Vs. Manual Modeling …………….........................................79 


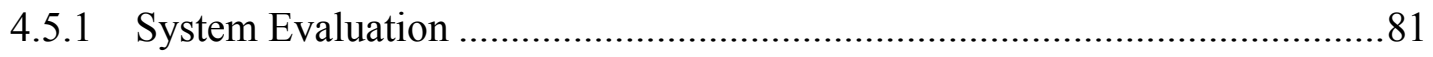

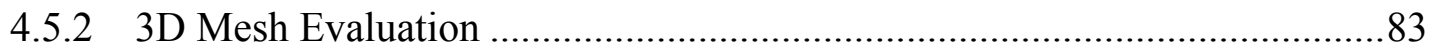

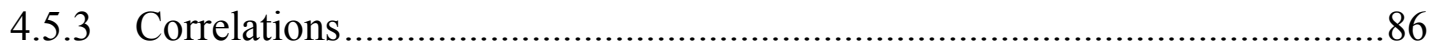

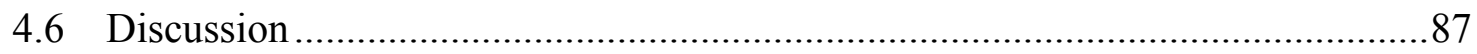

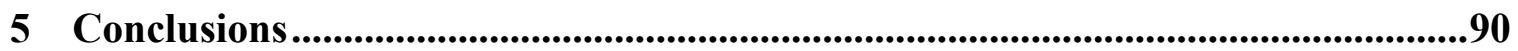

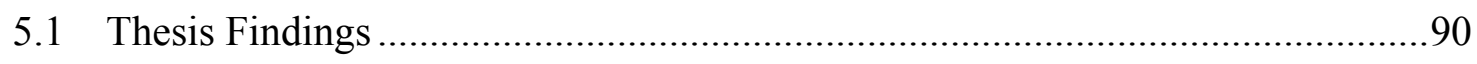

5.2 Limitations \& Future Work ....................................................................... 91

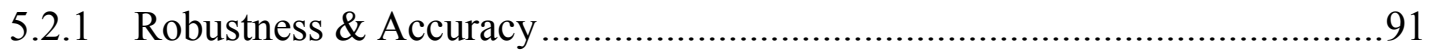

5.2.1.1 Pixel-Based Tracing ..................................................................... 91

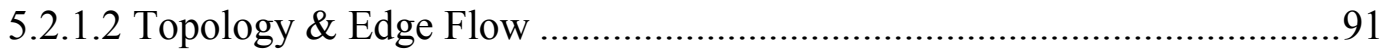

5.2.1.3 Upper Torso Structure...............................................................91

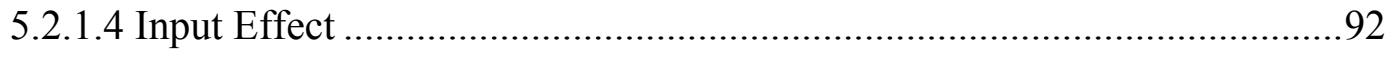

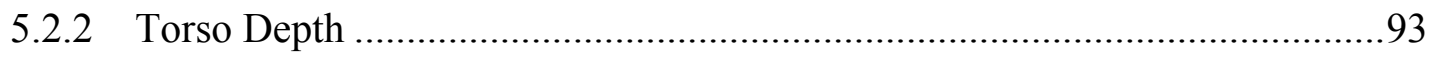

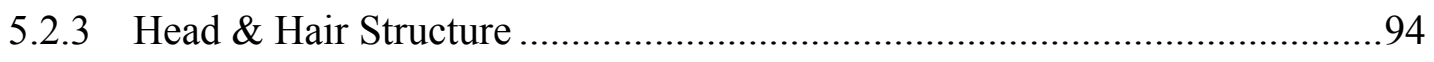

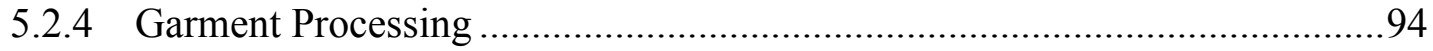

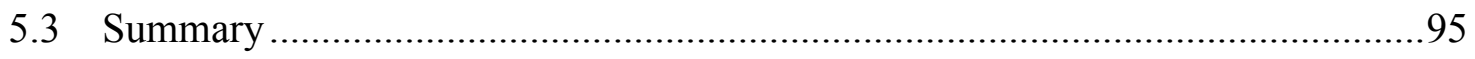

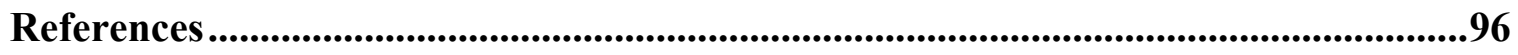

Appendix A: Permission to Use Copyrighted Material Form .....................................103

Appendix B: System Results .......................................................................................104

Appendix C: Consent Form ..........................................................................................108

Appendix D: Evaluation \& Feedback Questionnaire ..................................................110 


\section{List of Figures}

Figure 1.1: A character mesh and a head mesh in different levels of detail .......................1

Figure 2.1: Buchanan et al. [51] System Results ........................................................ 19

Figure 2.2: Bessmeltsev et al. [54] system results ....................................................21

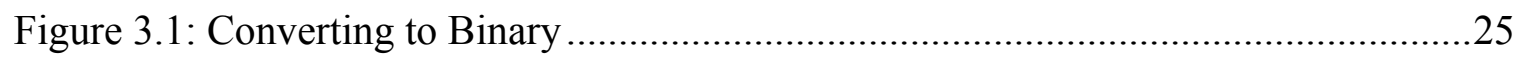

Figure 3.2: The outline result (in green) of the character, using Potrace [52] .................26

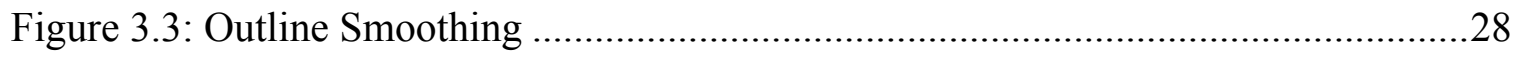

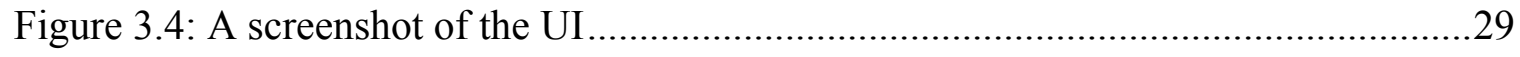

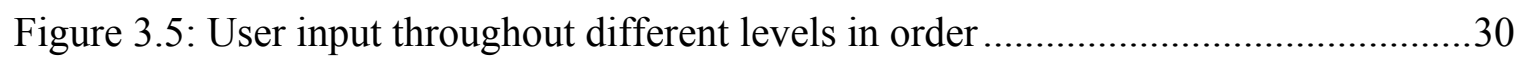

Figure 3.6: Labeled Input Points (User Perspective) ............................................... 32

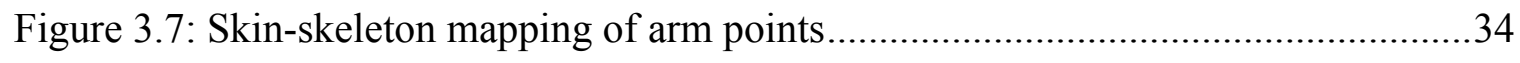

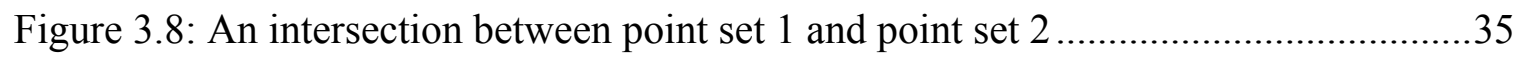

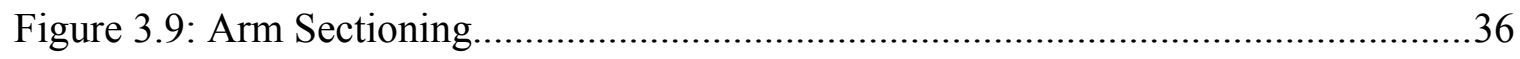

Figure 3.10: The leg axes, horizontal ankle lines, and intersection points......................37

Figure 3.11: Crotch Point \& Pelvis Corners ......................................................... 38

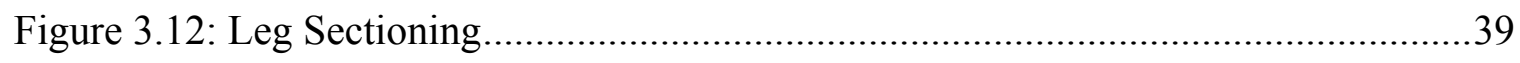

Figure 3.13: Limb Axis Correction Process ...............................................................40

Figure 3.14: The neck points and their horizontal intersections with the outline.............42

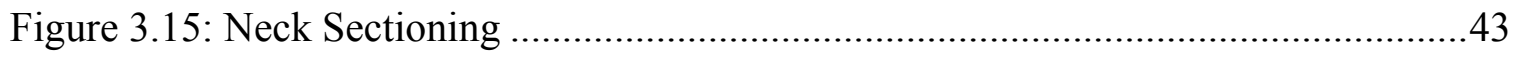

Figure 3.16: A summary of calculated dimensions of the head ...................................44

Figure 3.17: Eight section lines vertically dividing the head.....................................46

Figure 3.18: Underarm \& Chest Line detection....................................................48 
Figure 3.19: The first method of forming the clavicle line .50

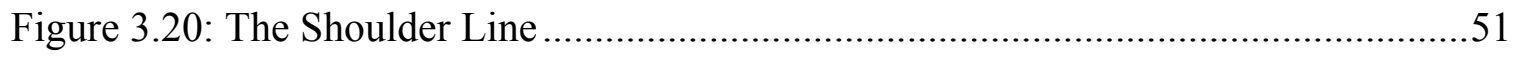

Figure 3.21: Five torso lines formed between the chest and thigh lines.........................52

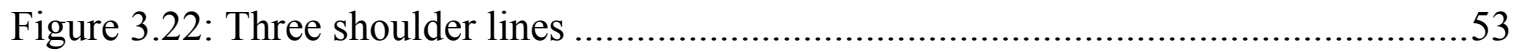

Figure 3.23: A complete 2D blueprint of the sketched character ...................................54

Figure 3.24: Head section lines and neck top line in front and side views......................56

Figure 3.25: Polygon surfaces constructed using 2D coordinates and orientation, as well as depth data. .61

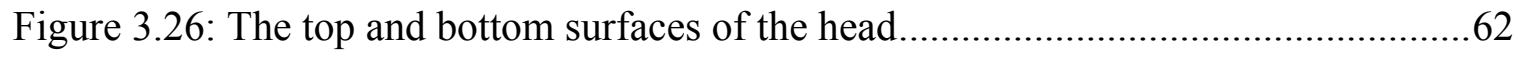

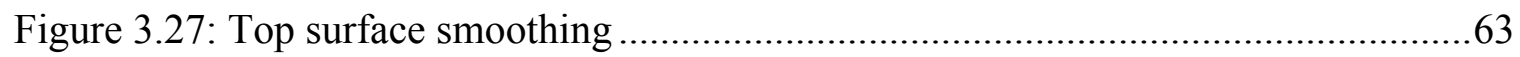

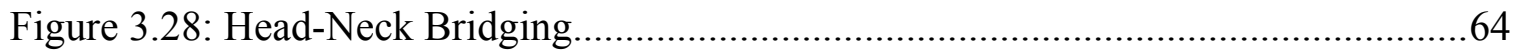

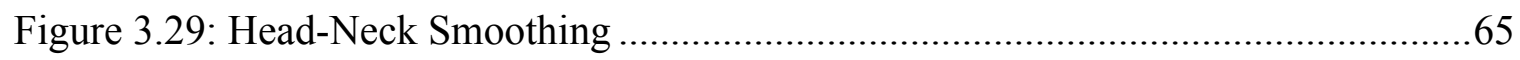

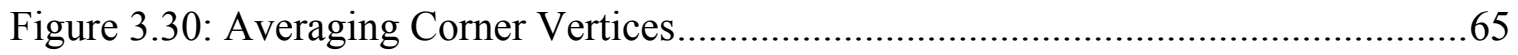

Figure 3.31: Head mesh before (left) and after (right) smoothing................................66

Figure 3.32: Torso Depth Adjustment (illustration in top view) ..................................67

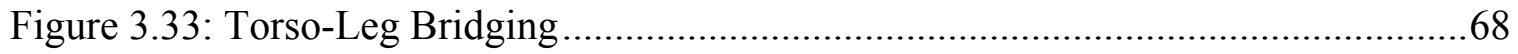

Figure 3.34: Torso-Leg Smoothing. Smoothing in (B) is only applied to front vertices...69

Figure 3.35: The hips before and after smoothing (side view) ..................................69

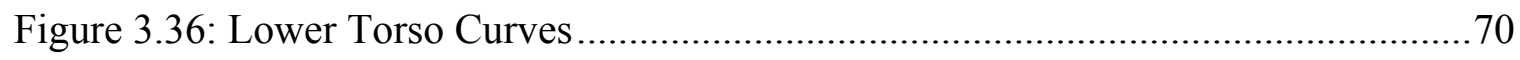

Figure 3.37: Lower Torso Restructuring in front (left) and back (right) .......................72

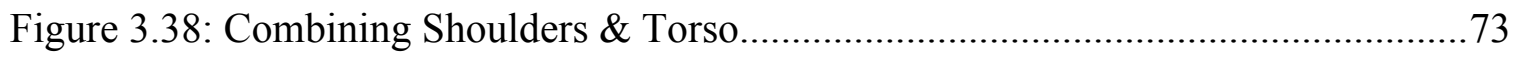

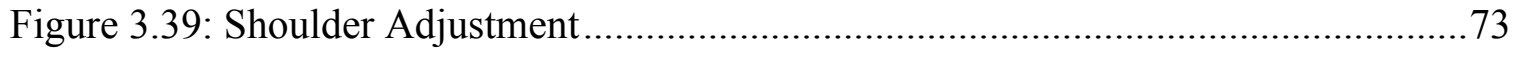

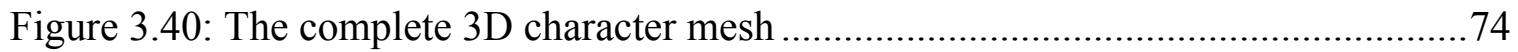


Figure 4.1: Users' 3D Modeling Experience .................................................................78

Figure 4.2: Users' estimated time to manually create the same basic geometry created by the system. .79

Figure 4.3: Users' preferred method to create the $3 \mathrm{D}$ basic geometry of the character.....80 Figure 4.4: Users' preference between automatic and manual modeling if they take the same amount of time 81

Figure 4.5: Users' evaluation of sketch and input requirements ........................................82

Figure 4.6: UI learning/interactivity \& speed evaluation ...............................................83

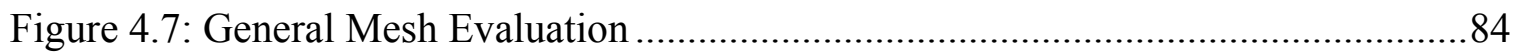

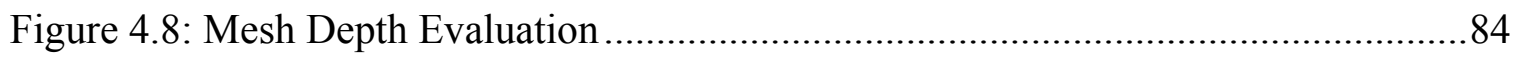

Figure 4.9: Users' willingness to use the automatically generated mesh...........................86 


\section{List of Tables}

Table 3.1: Width and Height ratios given for each head section line (Figure 3.17) .........46

Table 3.2: Depth ratios given for each head section line (Figure 3.24) ..........................57

Table 3.3: Upper \& Lower Curve Rotation Angles (Front)............................................71

Table 3.4: Lower Curve Rotation Angles (Back) ............................................................ 71

Table 4.1: Average \& median of mesh geometry \& topology evaluation ( 1 to 5 scale) ...85

Table 4.2: Pearson Correlation of character modeling experience with mesh evaluation

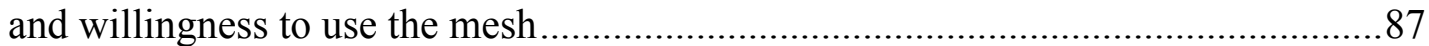




\section{List of Acronyms}

\begin{tabular}{l|l} 
Acronym & Definition \\
\hline 2D & Two-Dimensional \\
AI & Three-Dimensional \\
CCW & Counter-Clockwise \\
CW & Clockwise \\
HCI & Human-Computer Interaction \\
Poly & Polygon \\
Quad & Quadrilateral \\
SBM & Sketch-Based Modeling \\
UI & User Interface
\end{tabular}




\section{Introduction}

Three-dimensional (3D) modeling of human characters is a vital part of the entertainment industry that includes film and video games [1]. Building a 3D character commonly starts by blocking out the basic shape of the character and ends by reaching the level of detail desired for that character [2] (Figure 1.1). This is the most important part of the character creation process because it affects every process that follows (i.e. texturing, rigging and animation). Depending on the required level of detail as well as the 3D modeler's skills, character modeling can be complex because it is constrained by professional guidelines, which 3D modelers are expected to follow to ensure suitable results [3].

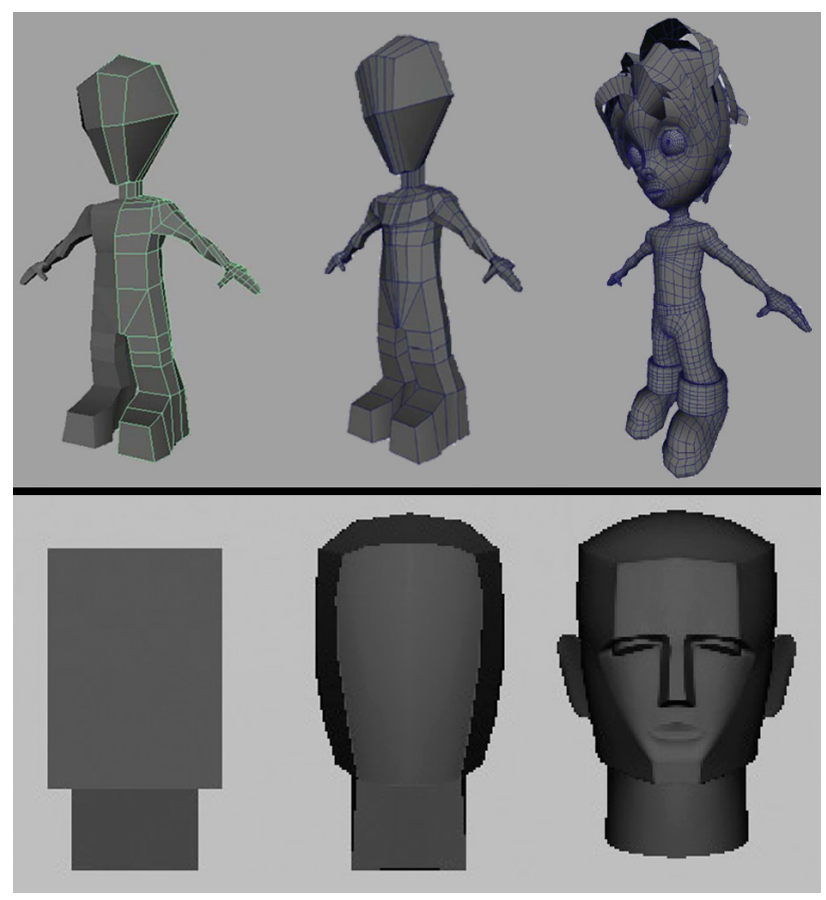

Figure 1.1: A character mesh (top) [3], and a head mesh (bottom) [4] in different levels of detail 
To organize and facilitate this complex process to the user, the common rule of building user interactive 3D modeling tools is user-centric design [5], where producing an intuitive and easy-to-use user interface (UI) is the main goal [6]. However, despite the popularity and increasing use of 3D software, such as Autodesk Maya ${ }^{1}$, Autodesk 3DS $\mathrm{Max}^{2}$ and Blender ${ }^{3}$, both experts and non-experts of the field consider these tools complex and difficult, and still demand better interfaces [7]. Thus, 3D production studios often integrate scripts to minimize the software's complexity for modelers, by automating repetitive and complicated tasks, and providing simpler UIs [8][9]. This enhances the 3D production pipeline because technical knowledge and complex functions are delegated to software engineers so that better UIs are created for 3D artists [10].

Moreover, according to Alhashim [11], novice 3D modelers find that using existing shapes to model a complex mesh is easier and less tedious than starting the process from scratch. This has led to the emergence of sketch-based modeling (SBM) techniques that strive to find efficient and accurate approaches to automate parts, if not all, of the modeling process.

\subsection{Sketch-Based Modeling}

SBM is the process of automatically creating a 3D model based on one or more sketches of the object to be modeled. While there are currently many systems designed for the purpose of SBM, Kazmi et al. [12] divide the types of 3D modeling applied by these systems into two categories: organic modeling using deformable meshes, and non-

\footnotetext{
1 http://www.autodesk.com/products/maya/

2 http://www.autodesk.com/products/3ds-max/

3 https://www.blender.org
} 
organic modeling, which focuses on mechanical and architectural modeling that is, alternatively, done using computer-aided design (CAD) systems.

SBM systems generally share the same pipeline, which consists of acquisition, filtering and interpretation [12]. There are two sketch or image acquisition methods that distinguish between different systems. The first method is interactive sketching, used to dynamically construct and modify the 3D model using the system's UI [13][14][15][16]. In these systems, users draw basic shapes which are immediately transformed into 3D meshes, and then use the system's offered features to manipulate a mesh in different ways like merging, cutting through or scaling.

The second acquisition method is preparing sketches or images in advance and then feeding them to the system for processing [17], although some systems that use this method still implement user interactivity with the input to emphasize or add details that the system requires from the sketch [18].

Filtering is the process of refining the input image or sketch to "de-noise" input details when necessary. Filtering examples include smoothing sketch strokes, deleting unnecessary ones, and improving input resolution. Filtering allows the system to result in more accurate interpretations of the input, the third and last phase of the processing pipeline, which is responsible of transforming the 2D input into a $3 \mathrm{D}$ mesh.

In summary, we introduced the concept of SBM, its different approaches, and its processing pipeline. Readers can refer to surveys on SBM for further information [7][19][20][12][21]. In Chapter 2, we discuss some of the SBM systems that have been a great contribution to the field, and then we focus on the ones that are closely related to our proposed methodology, which focuses on modeling human characters. 


\subsection{Problem Description}

Despite the increase of SBM systems, only a few focus on human character creation, while the rest create algorithms that model arbitrary objects. The latter is problematic because a character's hierarchical structure is modeled differently from simpler objects, and a generic algorithm may sacrifice the accuracy of that structure. Moreover, most systems do not focus on creating character meshes that conform to common modeling methods used by professionals and taught to novice modelers. As a result, they produce meshes that are not yet supported in film and game production.

On the other hand, based on our research, there are very few, if any, SBM systems available, to novice modelers in learning environments, which facilitate the character modeling process by automatically producing the basic geometry of a mesh, based on a sketch, and in a short amount of time. Most of the available systems are databased, such that a generic template character is used to start the process. The problem with these models is that they are usually highly detailed and customizing them may take longer than working with low-poly meshes.

\subsection{Proposed Method}

We propose a SBM method that aims to facilitate the process of 3D human character modeling by automating the construction of the character's basic geometry using a single front-view sketch and minimal user input. The objective of our system is to help novice modelers build human characters faster by automating the initial phase of character modeling, to reduce the complexity of building the character from scratch. The 
basic mesh produced by our system is a low-poly mesh that represents the basic shape of the character.

We created a simple UI to display the sketch and allow the user to input required points on the character. The UI is integrated in the Autodesk Maya workspace, where the 3D mesh is constructed. Our proposed methodology strives to conform to standard modeling guidelines, including suitable geometry and topology, to construct a mesh that can be used as the base of a game or film character.

Our system follows the SBM processing pipeline: it starts by reading and filtering a $2 \mathrm{D}$ character sketch. Next, it requires users to input a few important points on the sketch that help speed up the modeling process and increase accuracy of the model. Finally, using the sketch and user input, the system displays a low-poly mesh that matches the shape and proportions of the sketched character. Each step of the process is explained in detail in Chapter 3. Meanwhile, in order for the reader to better understand our proposed approach, we list below some professional modeling guidelines that we follow to model the character.

\subsubsection{Modeling Guidelines}

One of the recommended modeling techniques that we follow is to limit the polygon type of the mesh to quadrilaterals (quads) [4]. This is considered good practice because in 3D software like Autodesk Maya, quad meshes result in better deformations. In addition, subdividing quads is the most consistent as opposed to subdividing triangles and other non-quad polygons. Finally, when the character model is smoothed, non-quad smoothed meshes often produce anomalies and behave unpredictably. 
In addition, character modelers should maintain two important characteristics: clean geometry and good topology [3]. Koussa and Koehl [22] define geometry and topology as follows:

- Geometry is the representation and description of the faces, edges and vertices in terms of their shape and coordinates

- Topology is the representation of associations or connectivity relationships between faces, edges and vertices

Accordingly, as Patnode [3] recommends, to keep a clean geometry, modelers should unify the polygon type across the entire mesh. He leans towards the use of quads for the geometry, for the same reasons mentioned above in this subsection.

Second, to maintain good topology, Patnode recommends evenly spaced polygons, which is achieved by making sure polygons across the mesh have roughly the same size, especially at the block phase. Consequently, subdividing the polygons, when increasing the level of detail of the character, is done smoothly and predictably. In addition to smooth subdivision, evenly spaced polygons also make the character model easier to texture, rig and animate. Another good topology practice is ensuring a proper flow of edges and edge loops across the mesh, especially around areas combining two body parts together, such as the neck, shoulders and hips.

To summarize, we gathered a few, but important, modeling guidelines and techniques recommended by professional modelers, which helped us create our automatic modeling algorithm. We reflect on these techniques in Chapter 3 when we discuss our method. Meanwhile, in the rest of this chapter, we list our research questions and contributions. 


\subsubsection{Research Questions}

In this research, the main questions that we wanted to answer were the following:

- How would 3D modelers, of different experience levels, evaluate the character mesh produced by our system, in terms of structure, dimensions, geometry and topology?

- Will our system speed up the initial process of blocking out the basic geometry of the character? If yes, are 3D modelers actually concerned about the speed of such system as much as they may be concerned about the accuracy of its results?

- Is the system's interactive UI considered intuitive and easy to use?

- Would 3D modelers use our system to block out the character mesh?

By testing our system with different character sketches, and conducting a user study experiment to get feedback on user interaction experience and evaluation of the system's results, we were able to form our conclusions and speculate the answers to those questions.

\subsection{Research Contributions}

Our system aims to facilitate the character creation process to novice modelers. To ensure that we meet this objective, our system is built as a script that works with one of the most popular 3D software used in character modeling: Autodesk Maya. This way, it provides a familiar environment for modelers, and constructs a 3D model that conforms to the geometrical constraints of common 3D software, bringing the SBM concept closer to the standards of an industry that has not yet to give its full support to SBM systems, due to limited functionality and non-standardized techniques [20]. In addition, we 
introduce an original and simple UI that reads and dynamically displays user input on the sketch, and it visually summarizes its $2 \mathrm{D}$ processing results to the user.

Furthermore, as Habbecke and Kobbelt [10] state, it is proven that it is much easier to fix a mesh that is still in block phase than to fix a fully detailed mesh. That being said, our system produces a low-poly mesh with minimal level of detail, and with the ambiguity that comes with building a 3D mesh from a single 2D sketch, there is always room for mesh inaccuracies. Therefore, by producing a simple geometry, our system strives to minimize the time it may take modelers to fix wrongly constructed parts of the mesh.

Our system also processes the sketch and constructs the 3D mesh in only a few seconds. We were able to compare our system's processing time with some related systems, while we failed to retrieve such data from the rest. We can't claim that our system generates a mesh faster than all previous works, but based on our research, a SBM system generating $3 \mathrm{D}$ geometry in a few seconds is relatively fast.

Moreover, compared to similar systems (discussed in Chapter 2), our system focuses more on the effort to produce the geometry and topology details that conform to professional guidelines to model human characters for movies and video games [3], which includes combining body parts in a suitable manner to produce relatively accurate edge flows in joined areas.

Finally, we introduce novel and simple approaches in 2D outline smoothing, 2D skin-skeleton mapping, limb axis correction, depth estimation and mesh smoothing, which we explain in our Methodology chapter. 


\subsection{Thesis Organization}

In the next chapter, we discuss the main categories of SBM and the notable systems that have contributed to the field. Then, we explore, in further detail, systems that are closely related to ours, by discussing their approaches, results and how they differ from our methodology.

In Chapter 3, we introduce our methodology in detail. We introduce the development environment and the tools used to build this system. Next, we discuss sketch requirements and the pre-processing phase of the methodology. After that, we present the system's UI and input requirements. Finally, we discuss in depth the automatic $2 \mathrm{D}$ and $3 \mathrm{D}$ processes implemented in our system to construct and smooth the 3D mesh.

In Chapter 4, we explain the user study experiment we conducted to get feedback from 3D modelers on their user experience with the system as well as the evaluation of the results. We discuss requirements, methodology, results and analysis of the user study.

Finally, in Chapter 5, we discuss our thesis findings and speculated answers to our research questions, and we explain the limitations as well potential improvements and future work. 


\section{Literature Review}

\subsection{Background}

SBM systems have started to emerge since the mid 1980s. The main objective of which was to help 3D modelers by creating automatic sketch interpretation systems [12]. Since then, the research area of SBM has been booming very quickly with novel approaches and techniques. Researchers have focused on the important aspects of SBM, including creating intuitive UIs [19], solving sketch interpretation inaccuracies, and creating 3D manipulation tools to deform and modify the 3D models [20]. Those aspects gather researchers from different domains including human-computer interaction (HCI), computer graphics and artificial Intelligence (AI).

SBM systems can be divided into four categories: single-view, multiple-view, data-driven and curve network systems. Single-view systems take as input one sketch or image of a specific or arbitrary angle, that the system interprets into a 3D model. Acquiring the single-view sketch can be through interactive sketching or reading an already sketched or prepared image. Examples of single-view systems include Teddy [13].

Teddy is considered the pioneer of interactive sketching UIs that focus on 3D free-form design [12], although it produced only simple models and provided limited features. Other systems that followed the footsteps of Teddy and further contributed to simple interactive free-form modeling techniques include SmoothSketch [23], BlobMaker [15], FiberMesh [14], and the systems proposed in [24], [25] and [26]. 
There are other single-view system categories besides free-form modeling. For example, Entem et al. [17] created a system that models 3D animals using side-view sketches by inferring occluded parts and depth information as well as assuming symmetry of all body parts. Another example is Life-Sketch [27], a system that reads the outline of a simple sketch and uses Delauney Triangulation to triangulate the outline shape and infer a chordal axis (or skeleton), used for 3D construction and animation.

Another important single-view system is introduced by Gonen \& Akleman [28], which constructs a "mostly" quad-based model based on curve partitioning and classification of a sketch. It is based on a simple yet innovative methodology that produces clean geometry and suitable topology of non-complicated shapes.

Multiple-view systems are the ones that take as input more than one sketch or photo, to construct the 3D model. Rivers et al [29] propose a notable multiple-view system, which focuses on modeling man-made objects. Viana et al. [30] propose another notable approach to create 3D models from multiple-view concept arts, which focuses on producing high quality objects at a low cost.

Data-driven systems are SBM systems that use databases of 3D templates to construct the final 3D model [31]. Data-driven systems have different approaches. One approach takes as input a sketch and tries to match it with a 3D template model in the database. After it finds its match, the system then tries to transform that template model to exactly match the shape of the given sketch. There are a lot of systems that use this approach, especially the ones that focus on human character modeling, which we discuss in the next section. Other considerable data-driven systems that focus on simpler objects 
include parameterized-object modeling by Yang et al. [32], photo-inspired modeling by Xu et al. [33] and sketch-based model retrieval by Li and Johan [34].

Curve Network systems are another important category in the SBM field, and a significant amount of research has been conducted in this category. 3D curve networks are $2 \mathrm{D}$ sketches that represent the 3D structure of an object. Unlike other sketches, 3D curve networks simulate the depth of the sketched object by drawing a network of curves that include every detail of the object's silhouette. In other words, these curves also include the parts of the object that, otherwise, would be occluded if drawn as a normal sketch.

Curve Network systems are an important SBM area of research, whose processing differs from regular sketches, which has attracted CAD system developers [35]. As mentioned in the previous chapter, $\mathrm{CAD}$ systems provide $3 \mathrm{D}$ modeling tools for design purposes in areas like architecture, industrial design and manufacturing. Thus, similar to any type of 3D modeling, CAD system artists start by sketching their designs and then using them to create 3D models. However, the approach of using SBM systems to automate $\mathrm{CAD}$ modeling differs from other modeling purposes because the more natural approach of sketching for CAD systems is using 3D curve networks [36]. Some notable SBM systems that are designed for CAD purposes include BendFields [37], True2Form [38] and Digital clay [39].

In short, we briefly discussed some of the categories and related works of the SBM field that are important to grasp as part of the literature review of this thesis. In the next section, we focus on human character modeling, where we introduce the systems whose methodologies are closely related to ours. 


\subsection{Related Systems}

As mentioned earlier, the research on SBM has been increasing lately. However, there are relatively fewer systems that focus on developing 3D human characters, and the majority of these systems are data-driven, relying on databases of 3D human models to start the model creation process [31].

On the other hand, a big percentage of existing approaches are multiple-view systems that use more than one view of a character to create a corresponding 3D model. While we would like to focus only on the existing systems that, similar to our methodology, use a single human character sketch and are not data-driven, we will include, in this literature review, other systems whose methodologies are relevant to our method in any of its important aspects.

\subsubsection{Data-Driven Systems}

In data-driven systems like [40], [41], [31], [42] and [43], the 3D human character is constructed using one or multiple 2D views of the character, some user interaction, and a database of 3D template models.

Zhang et al. [40] created a system that transforms a template model of a human character based on anthropometric measurements, taken as user input or captured from two views (front and side) of a person using Microsoft's Kinect. Anthropometric measurements consist of physical information, including proportions and dimensions, about the human body [44].

The system in [40] sets a fixed database of example human models that share a standard pose, but differ in size and proportions. In each model, a list of 30 
measurements, containing length and girth measures of body parts, is extracted. These 30 measurements are based on rules of standard human body dimensions and body mass distribution. After that, the system applies a correlation analysis and an interpolation algorithm to examine the relation and map between the different measurements of the template models.

This system takes as user input a partial set of the desired anthropometric measurements for the goal model [40]. It then uses the calculated correlation and map to estimate the 30 measurements from the given input and to generate an initial 3D model. The system optimizes the model using constraining feature curves to conform to user input while making sure it maintains its initial features that are taken from the correlation and map of the example models.

The methodology in creating a 3D human model in [40] is thorough and produces good results. However, it differs from our approach because, not only it uses a datadriven approach and multiple-view images, but it is also dependent on standard anthropometric measurements, in terms of the lengths and distances between body segments. This does not allow for the freedom to produce non-realistic proportions of characters, which our algorithm aims to achieve.

In another approach, Johnston et al. [41] propose a system that reads a front-view concept sketch of a human character, detects the $2 \mathrm{D}$ pose of the character and transforms it into a $3 \mathrm{D}$ pose, and finally produces a model using a template human model, the estimated body proportions and the 3D pose of the sketched character. The system adapts algorithms that include [45] and [46], to detect the 2D pose and estimate the 3D pose of the character. In addition, user interaction is allowed to fix poorly detected poses. 
To find the closest template model that matches the concept sketch, the system generates samples of models. It then transforms each sample from its default pose to the detected 3D pose and shape using inverse kinematics and linear skinning. Finally, it computes the weight of each transformed sample to find the best matching one.

The system in [41] distinguishes the character in a sketch, from the background, by assuming that the edge density of the character is much greater than the edge density of the background. In addition, the authors state that the concept sketches tested on the system are more of "hidden" 3D models represented in 2D, due to their highly detailed drawing, coloring and shading. This means that, unlike our approach, the sketches read by this system are expected to be in full detail rather than the original and simple reference sketches usually used in 3D modeling.

In addition, after transforming the size and proportions of sample models, results from different sketches still look somewhat similar due to the generically detailed face, hands, feet and body muscles, which is one of the main disadvantages of using 3D templates to create the basic geometry of a character.

The system proposed by Kazmi et al. [31] is a similar approach to [41]. It relies heavily on detailed contours of the sketch to transform the template model, and estimate the pose of the character. In addition, similar to [41], there is already enough detail in the generic mesh that makes it less flexible when it comes to producing basic meshes for different types of characters.

Finally, perhaps the most relevant data-driven system, to our approach, is the Virtual Human Sketcher (VHS) [42]. VHS is one of the oldest and basic sketched-based modeling systems, which produces a simple geometry of a human character from a 
transformed 3D template. However, VHS differs from our approach because it relies on user interaction in each phase of the development. In addition, although the geometry is simple and is more flexible to add varying details, the generic template produces very similar results every time, and the system restricts users to only one character drawing style that can be drawn in a different size and fat percentage. VHS, as demonstrated by the authors, may be more suitable for low-quality crowd modeling than, for example, modeling a main character in a 3D game or movie.

\subsubsection{Multiple-View Systems}

There are two types of multiple-view systems that create 3D human characters. The first type is the one that uses two views, usually the front and side of a sketched character, or a real person, to construct the model [47][48]. The second type is the one that uses a sequence of photos, which can be as many as 65 photos taken from different angles, of a character or any object [49][50]. The second type implements algorithms, including 3D point cloud construction, which differ from our approach. Therefore, we will not discuss them in this literature review.

An example of a two-view system is [47] that is developed for garment design and virtual fitting. The two captured photos of a person are used along with a template model to create the final human model matching the size and proportions of the person in the image. While the system produces a deformed model that is relatively proper for virtual garment fitting, the geometry and topology of the model is somewhat different from the professional guidelines proposed for movie and game characters [3].

Zhou et al. [48] later improved the virtual fitting system [47]. In [48], the system first processes the two views by extracting characteristic points from the person's 
silhouette and estimating body proportions. Next, using a template model and target body measurements, the system uses global and local free-form deformations to transform the template model into the desired shape. Global deformations transform the entire mesh at once, while the local ones focus on one body part at a time.

\subsubsection{Single-View, No-Database Systems}

\subsubsection{Fully Automatic Construction From Concept Art}

One of the notable systems that focus on constructing 3D characters from a single view image, without the use of template models, is the work done by Buchanan et al. [51]. This system reads a concept image of a character and uses the character's outline to extract a 2D skeleton, which is then used to produce the $3 \mathrm{D}$ model.

The system in [51] first uses the Potrace algorithm [52] to trace the outline of the character. Once the outline is identified, the system then creates a $2 \mathrm{D}$ skeleton using an adapted smoothing and merging algorithm. This algorithm consists of iterations of smoothing and encroachment steps that are ended with a merging step in the end. The smoothing part contracts the character outline towards the center by making each point on the contracted outline the average of its adjacent points that are not yet averaged, while at the same time conserving point density. The encroachment part of the process moves the points along the normal, and uses the character image complexity to produce a more accurate skeleton.

After finalizing the skeleton and before generating the 3D mesh, the system applies an additional step, which is estimating the orientation of the character from the concept image using local image complexity. The reason the system estimates the 
orientation is that it does not assume that the character is always in front view; it accepts off-centered images as well, and thus, it uses color weighting to estimate a more accurate orientation. After determining the orientation, the system starts to construct 3D mesh surfaces. The mesh is consisted of a set of arcs where each arc is based on a point on the outline and a center point on the skeleton. Finally, a cross-section is extruded along the skeleton to create the surfaces.

The system introduced in [51] is a novel approach and a major contribution in SBM that focuses on character modeling, and is a complete system that produces 3D characters from single-view concept images. However, similar to [41], this system relies on detailed and colored concept images for a more accurate skeleton creation process and orientation estimation. Therefore, we assume that using simple reference sketches, which we use in our system, can produce less accurate mesh results.

Moreover, the 3D construction process in this system results in a 3D surface that consists of half circles or "arcs" revolving 180 degrees around the skeleton, creating a different topology than the standard topology used in game and movie characters [53]. Figure 2.1 shows some of the results of the system. 


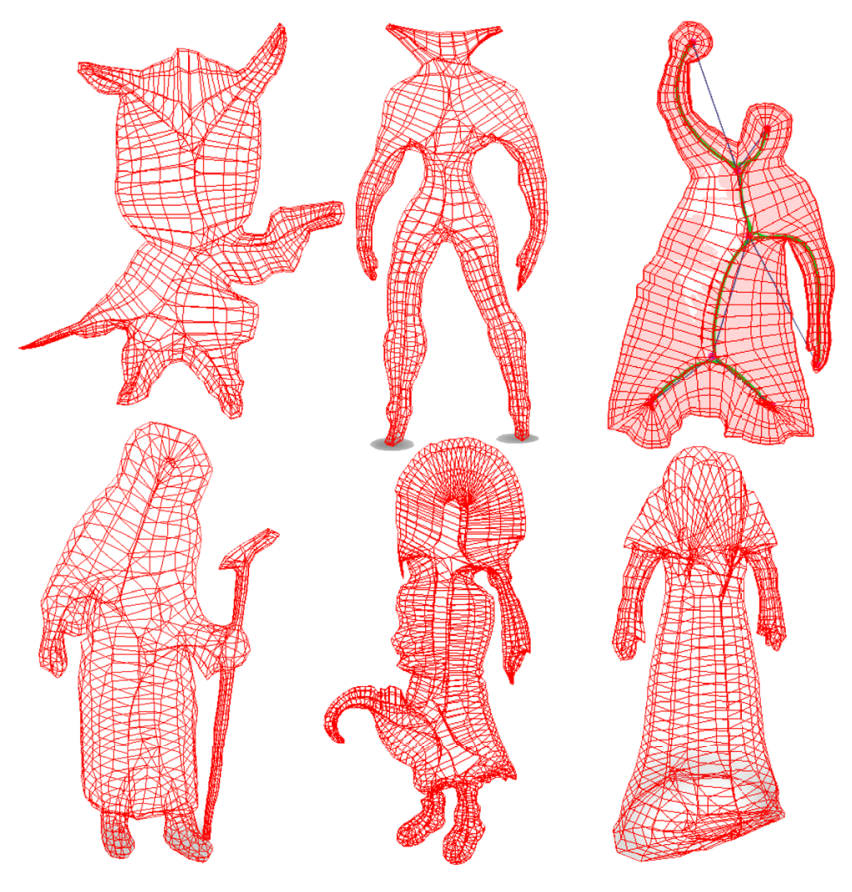

Figure 2.1: Buchanan et al. [51] System Results

\subsubsection{3D Skeleton-Based Construction of Cartoon Drawings}

Bessmeltsev et al. [54] created another notable work that is relevant to our methodology, but is capable of processing all types of cartoon characters. Their system integrates the use of a 3D skeleton to help define the depth of the character and eliminate the ambiguities of single-view modeling. The system starts its process by reading a cartoon drawing and a manually created 3D skeleton that represents the desired pose. After that, the system partitions the character outline, into body parts, based on the skeleton's joint-connected bones, using existing algorithms focusing on surface segmentation. The use of a 3D skeleton further helps the system when occlusions in the drawn character are present.

After each of the skeleton bones is fitted into the segmented parts of the character outline, a "curved skeleton" is estimated to better fit the shape of the character than a 
rigid skeleton, while ensuring that the symmetry of the character is maximized. After fitting the skeleton, the system starts to create $3 \mathrm{D}$ surfaces by revolving each surface about its 3D axis, which is basically a bone on the given skeleton. The final 3D model is always aimed to achieve optimized symmetry in its body parts, conform to the character outline and contours, and create a model whose side-view outline is continuity-persistent with the front-view outline.

This system avoids the disadvantages of automatically generating a skeleton that [51] faces, by having the skeleton manually created to match the character drawing [54]. Moreover, it more accurately estimates the depth and the occluded parts of the character based on that 3D skeleton. However, while the algorithm creates high-quality meshes, there are some topology issues around joined areas, which the system itself does not resolve, as stated by the authors.

In addition, the process of creating a 3D skeleton (or rig), for novice modelers, to generate the basic geometry of a character can be a daunting task, and it defies the objective of our system, which focuses on speeding up the modeling process without adding major tasks like creating a rig, for example. Figure 2.2 shows some results of the system and the topology issues in joined areas. 


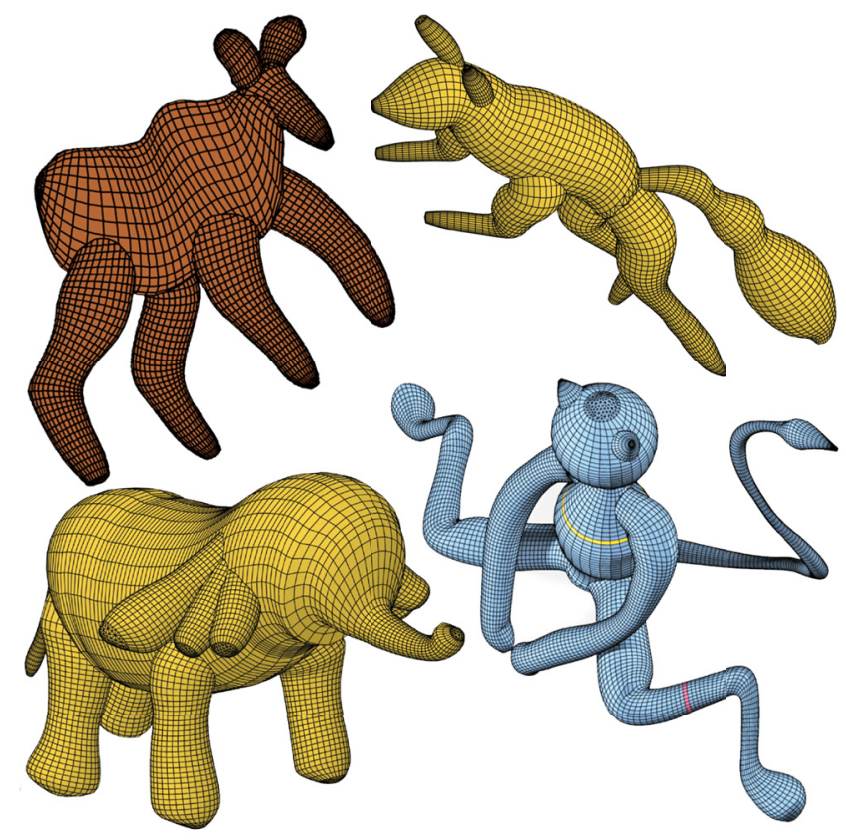

Figure 2.2: Bessmeltsev et al. [54] system results. The topology issues in joined areas are not solved by the system

After we discussed existing research on SBM systems that are relevant to our methodology, we introduce our system in the next chapter and explain in detail every phase of the process. Later in this thesis, we present our experiment with a user study to evaluate our system results and user experience, and we also include some test results of the system using different character sketches. 


\section{Methodology}

\subsection{System Overview}

To model the character's basic geometry, our system starts by automatically tracing and smoothing the outline of the sketched character. It then displays a simple UI that allows the user to input a few points on the sketch, locating important body joints. Then, using the outline and user input, it automatically derives additional joints, forming a rough skeleton and mapping between the outline and skeleton. As a result, a character blueprint is created and used for 3D construction. Finally, the system estimates the body's depth, constructs and attaches body parts, creating a low-poly 3D character mesh.

Throughout this chapter, we are going to explain each part of the system in detail, starting with outline tracing and smoothing. After that, we will present how the UI and user input are integrated to the system. Next, we will explain how additional information and outline-skeleton mappings are generated. Finally, we present how our algorithm estimates the depth of the character for each body part, and explain our approach to attach and smooth the 3D body parts.

\subsection{Development Environment}

This system was developed using Python 2.7. We used PySide's QtGui and QtCore libraries to create and display a dynamic UI and process user input. The Python script is built for Autodesk Maya; we use OpenMayaUI module to open the UI window 
in Maya, and we use Maya commands in Python to automatically develop the 3D mesh in Maya’s workspace.

\subsection{Sketch Requirements}

The system reads a digital image file that displays a sketched character. The character can be hand-drawn and then scanned to a digital file, or it can be digitally sketched. However, the final sketch has to be a pixelated image in a raster file format, such as JPEG or PNG.

To ensure that our system works as expected, it is assumed that the character sketch meets the following requirements:

1. The background of the sketch is white or very light color

2. The outline of the character is clear and not faded

3. A human character in front view, with the arms extended 45 to 90 degrees away from the body

4. The character has no loose garment or gear or other objects on them

5. Character's hair is chin-length or shorter

\subsection{Sketch Preprocessing}

When the system reads a sketch file, it converts it to a binary image in order to extract the character from the background of the image. To convert to binary, we use a simplified version of the K-Means Clustering Algorithm [55]. We divide the image into two clusters, background cluster and foreground cluster. Instead of randomly choosing our cluster centroids, we choose the background cluster centroid $c_{0}$ to be the lightest pixel in color value, which is the pixel with the largest $\mathrm{R}, \mathrm{G}$ and $\mathrm{B}$ values $r_{0}, g_{0}$, and $b_{0}$ 
respectively. In contrast, the foreground cluster centroid $c_{l}$ is the darkest pixel in color value, which is the pixel with the smallest $\mathrm{R}, \mathrm{G}$ and $\mathrm{B}$ values $r_{l}, g_{l}, b_{1}$ respectively.

After finding $c_{0}$ and $c_{l}$, we define a threshold $t$ that the system uses to decide which cluster each pixel of the image belongs to. Threshold $t$ is defined as follows:

$$
t=\left[\operatorname{SUM}\left(r_{t}, g_{t}, b_{t}\right) / 3\right] * 0.8
$$

where $r_{t}$ is the absolute difference between $r_{0}$ and $r_{l}, g_{t}$ is the absolute difference between $g_{0}$ and $g_{l}$, and $b_{t}$ is the absolute difference between $b_{0}$ and $b_{1}$. The created threshold sets a relatively large range of values for foreground pixel colors, to include most colors in the foreground cluster, while including only a limited set of colors, mainly whites and very bright colors, in the background cluster.

We run a test for each pixel to evaluate if that pixel belongs to the foreground cluster, and if that test fails, then it is added to the background cluster. The test is as follows:

For each pixel $p_{i}$, with color values: $r_{i}, g_{i}, b_{i}$, in the image, $p_{i}$ is considered part of the foreground cluster if and only if $r_{c}, g_{c}$, and $b_{c}$ are each less than or equal to $t$, where $r_{c}$ is the absolute difference between $r_{i}$ and $r_{l}, g_{c}$ is the absolute difference between $g_{i}$ and $g_{l}$, and $b_{c}$ is the absolute difference between $b_{i}$ and $b_{l}$. Otherwise, $p_{i}$ is added to the background cluster.

After iterating through all pixels, the system finally sets the color value of background cluster pixels to white, and sets color value of foreground cluster pixels black. Figure 3.1 shows a character sketch converted to binary. 

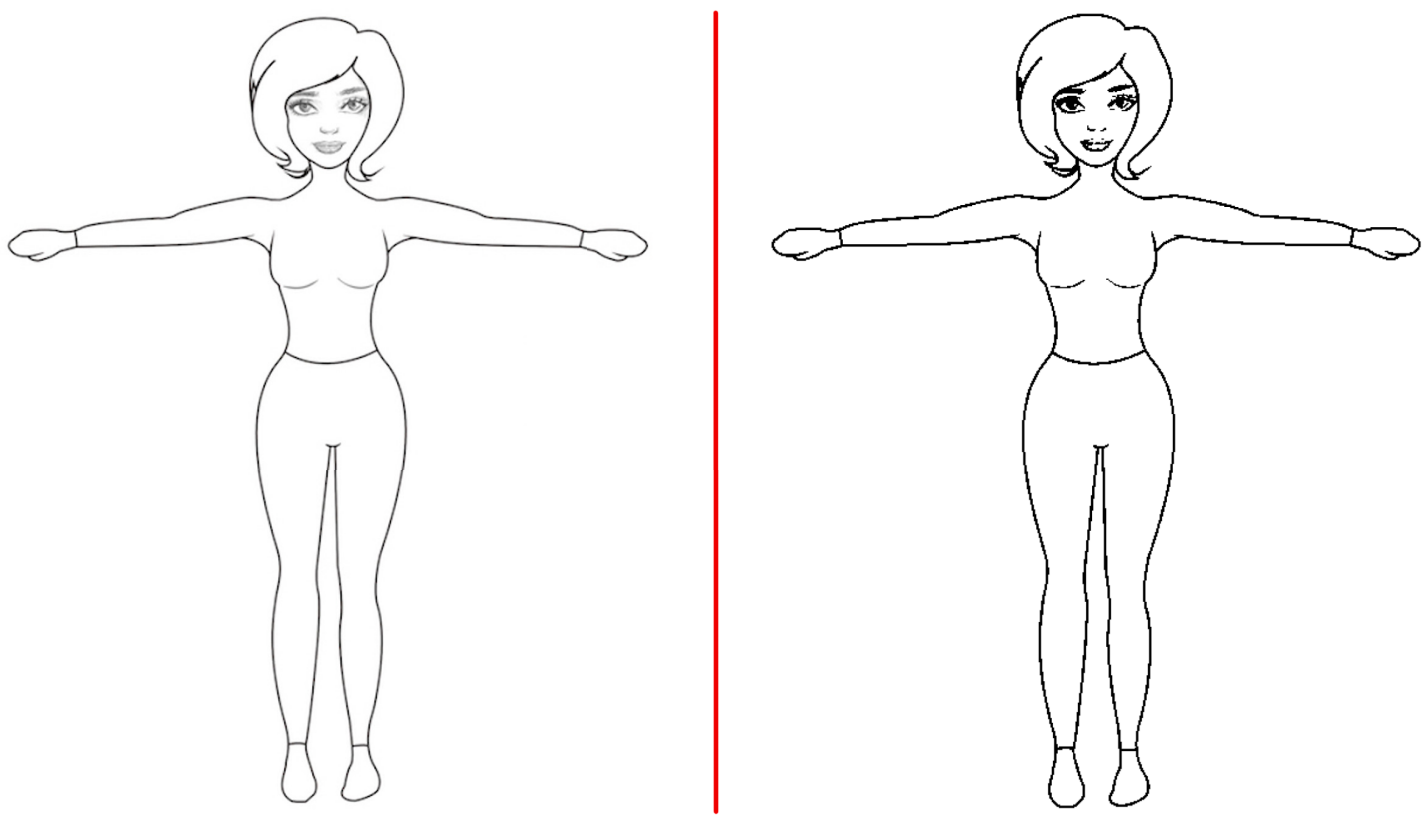

Figure 3.1: Converting to Binary. An original character sketch (left) is converted by our system to a binary image (right)

\subsection{Outline Tracing}

After converting to binary, the system traces the outline of the character sketch. Similar to Buchanan et al. [51], we use the Potrace Algorithm [52], which works on binary images. It traces the character by finding the top-leftmost foreground pixel and traveling its way around the character in counter-clockwise $(\mathrm{CCW})$ direction, tracing only the outermost pixels of the character. Finally, outline pixels are stored in an array, in $\mathrm{CCW}$ order, each with a unique index and 2D coordinates. Figure 3.2 shows a character outline traced by our Potrace implementation. 


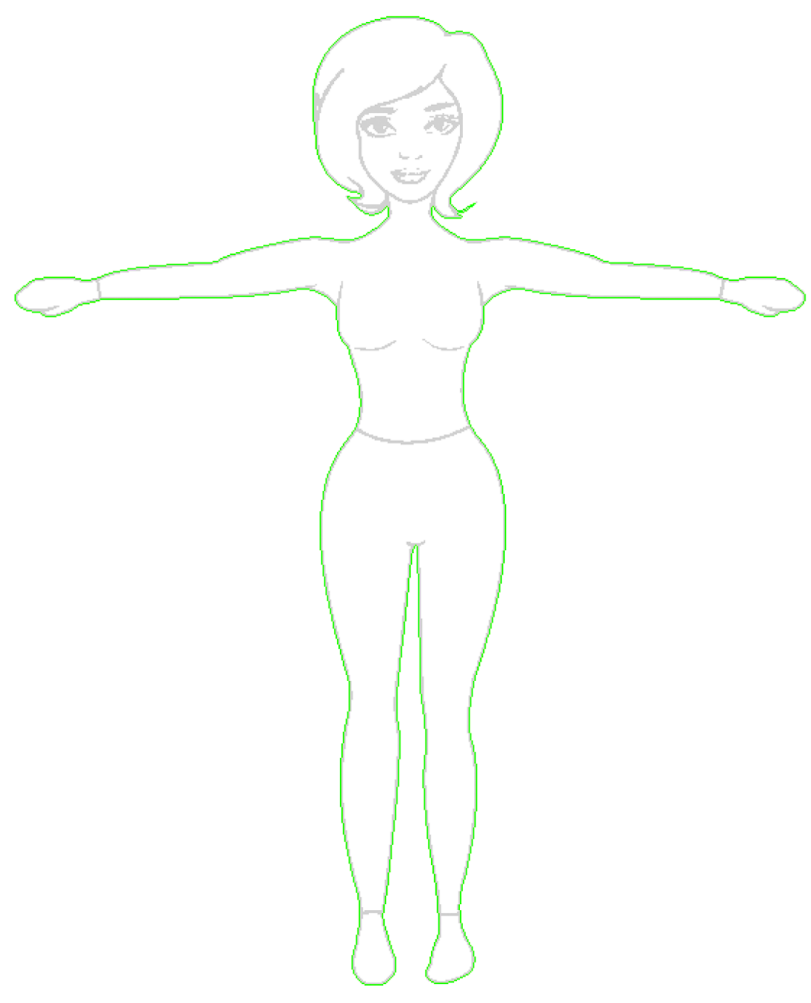

Figure 3.2: The outline result (in green) of the character, using Potrace [52]

\subsubsection{Outline Smoothing}

To reduce the side effects of "overtraced strokes" [56], and roughness of pixelbased tracing, we applied a smoothing algorithm to the character's outline. This algorithm is based on pixel directions and inspired by the feature detection algorithm introduced by Lin \& Wang [57]. Our system defines a pixel direction $d_{0}$, as the vector directed from a pixel $p_{0}$ to pixel $p_{1}$, where $p_{1}$ is adjacent to $p_{0}$ in the outline array. In this algorithm, we calculate $d_{0}$ and evaluate the change of direction within a range of four pixels from $p_{0}$ to $p_{3}$. If the angle of direction changes 90 degrees or more, from $d_{0}$ to $d_{3}$, then the group is candidate for smoothing. 
To avoid over-smoothing the outline, a candidate pixel group is smoothed if and only if among the direction vectors of that group, at least two vectors are identical; which means that the angle between those two vectors is zero degrees. This restriction prevents the system from smoothing narrow areas of the character outline, like between legs for example.

When a candidate pixel group $p_{0}$ to $p_{n}$, where $n$ equals 1,2 or 3 , is eligible for smoothing, the system removes the pixels between $p_{0}$ and $p_{n}$ from the outline, and connects $p_{0}$ and $p_{n}$ with a straight line. The reason why we chose four consecutive pixels to be the maximum pixel group size is because we do not want the system to mistake body features or important garment detail for "likely unnecessary" information that may be eliminated if smoothed. Figure 3.3 shows examples of smoothed pixel groups in two partial outlines. 

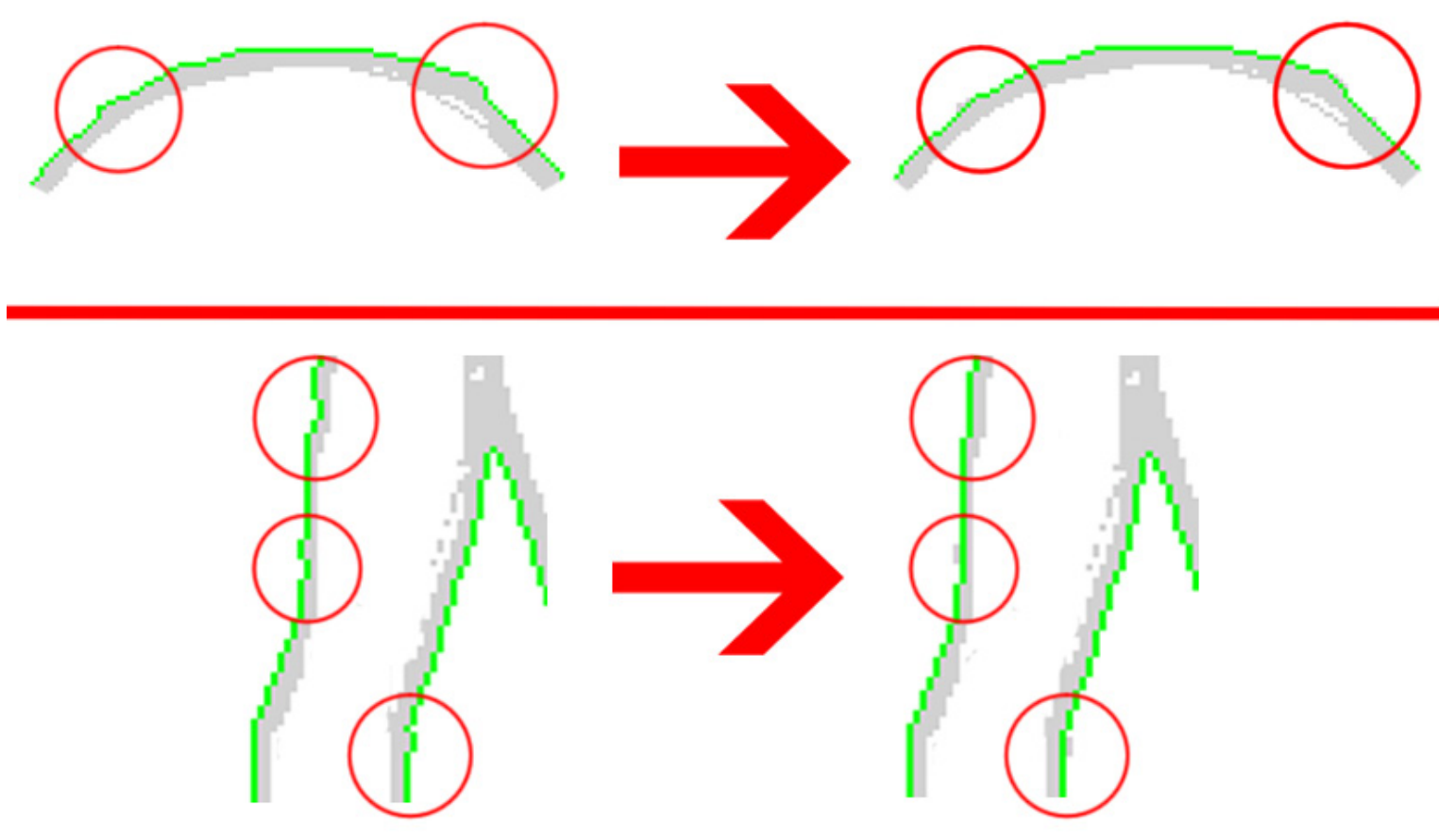

Figure 3.3: Outline Smoothing. Some pixel groups (circled) are shown before (left) and after (right) smoothing

\subsection{The User Interface}

The UI we designed to interact with the system is a simple window, displaying a color-faded character image and a visible traced outline of that character. To the right side of the image, the UI displays a side bar that contains necessary information like the current required task, a button to move to the next level, and mouse cursor coordinates. In addition, for each point the user inputs; the corresponding coordinates of that point are displayed. Figure 3.4 shows a screenshot of the UI. 


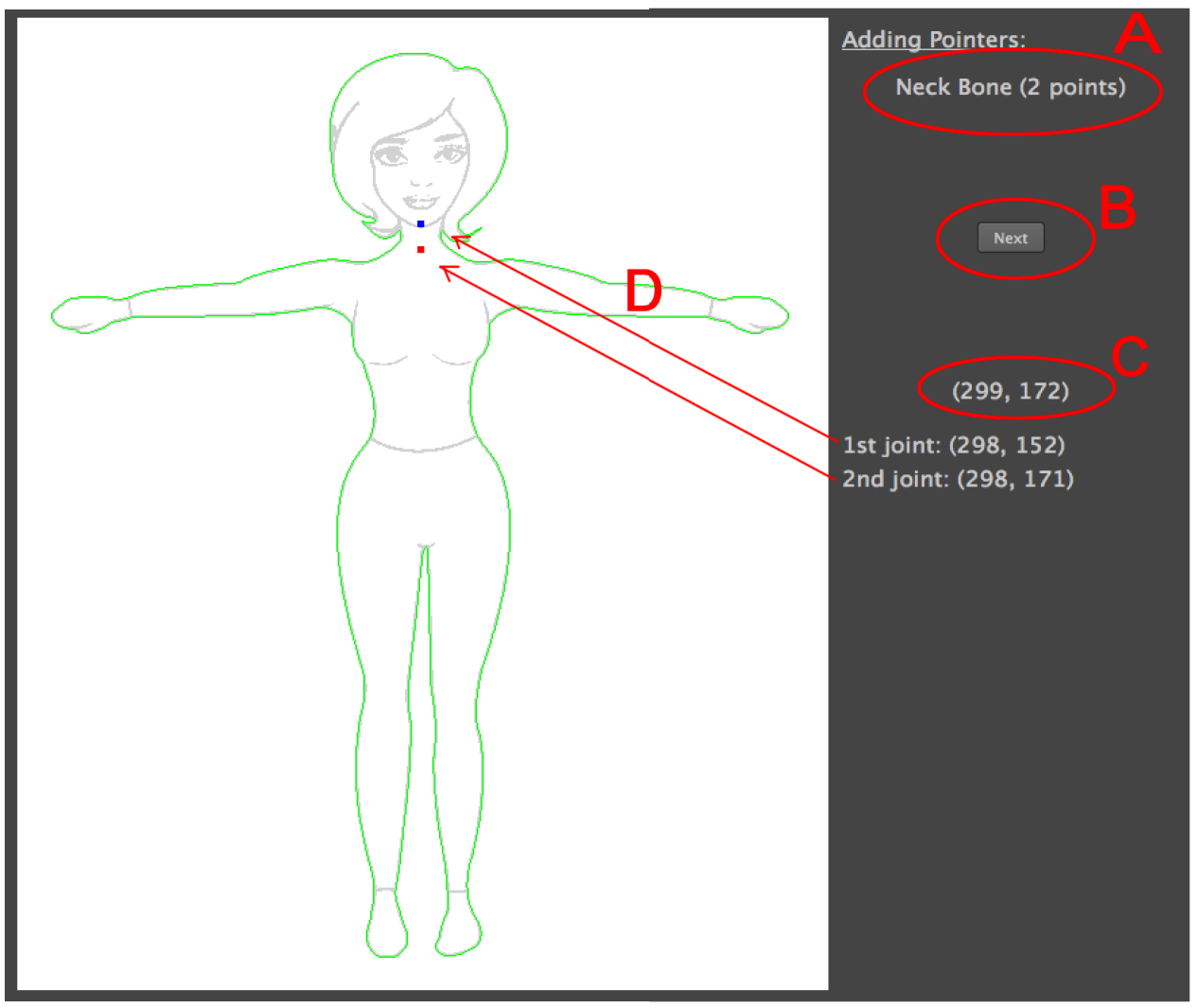

Figure 3.4: A screenshot of the UI. $A$ represents the current required task. $B$ is the button clicked to move to the next input level. $C$ represents current mouse curser coordinates, and $D$ is the corresponding coordinates of points input by the user

\subsubsection{User Input}

Users are required to input a total of 10 points. Each two points are on a separate input level. The first level is to locate the neck. Two points are required to estimate the start and end of the neck area. The second level is to roughly locate the biceps, with one point on each arm. Our algorithm expects the bicep input to be closer to the shoulder rather than being closer to the elbow. The third level is to locate the wrists, one point on each arm. The fourth level is to locate the hipbone (or pelvic bone), by inserting a point on the middle of each hip line. The fifth and last level is to locate the ankles with one point for each. 
Figure 3.5 shows an example of user input throughout different levels. The system does not allow the user to move to the next level until the required number of points for that level is fulfilled. To clarify that constraint, the system hides the "Next" button at a certain level until input is complete. In addition, at each level, the user can adjust coordinates of the point by using the keyboard arrow keys.
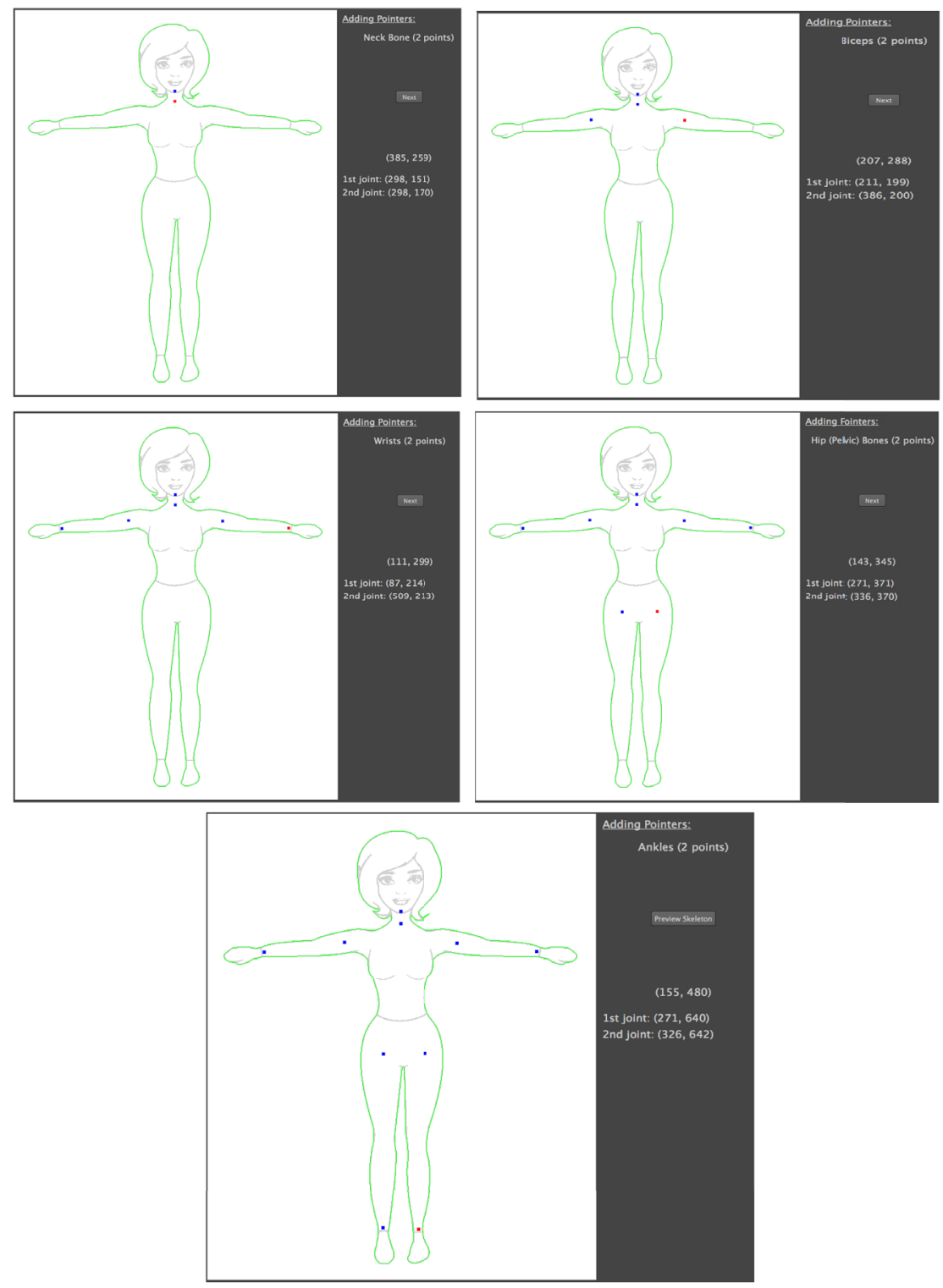

Figure 3.5: User input throughout different levels in order (left to right, top to bottom) 


\subsubsection{Input Processing}

After completing user input, the system organizes the 10 points in a data structure that represents a rough skeleton for the character. Because different input was separated in levels, we made it easy for the system to recognize the type of each input. For example, in level 3 , the system is aware that the current input is to locate wrists, and not ankles.

With point type recognition, the system only needs to distinguish between the two points of the same level. To do that, the system calculates the $(\mathrm{x}, \mathrm{y})$ coordinates of those points and labels them as the following:

- For biceps, wrists, hips and ankles, the left point is the point whose $\mathrm{x}$ value is smaller.

- For the neck, the top of the neck is the point whose y value is smaller.

The smaller $\mathrm{x}$ values are considered a left side from the user's perspective and not the character's, which is how the system labels body parts. Thus, we use this labeling notion throughout our methodology discussion. Figure 3.6 shows the final labeled points. 


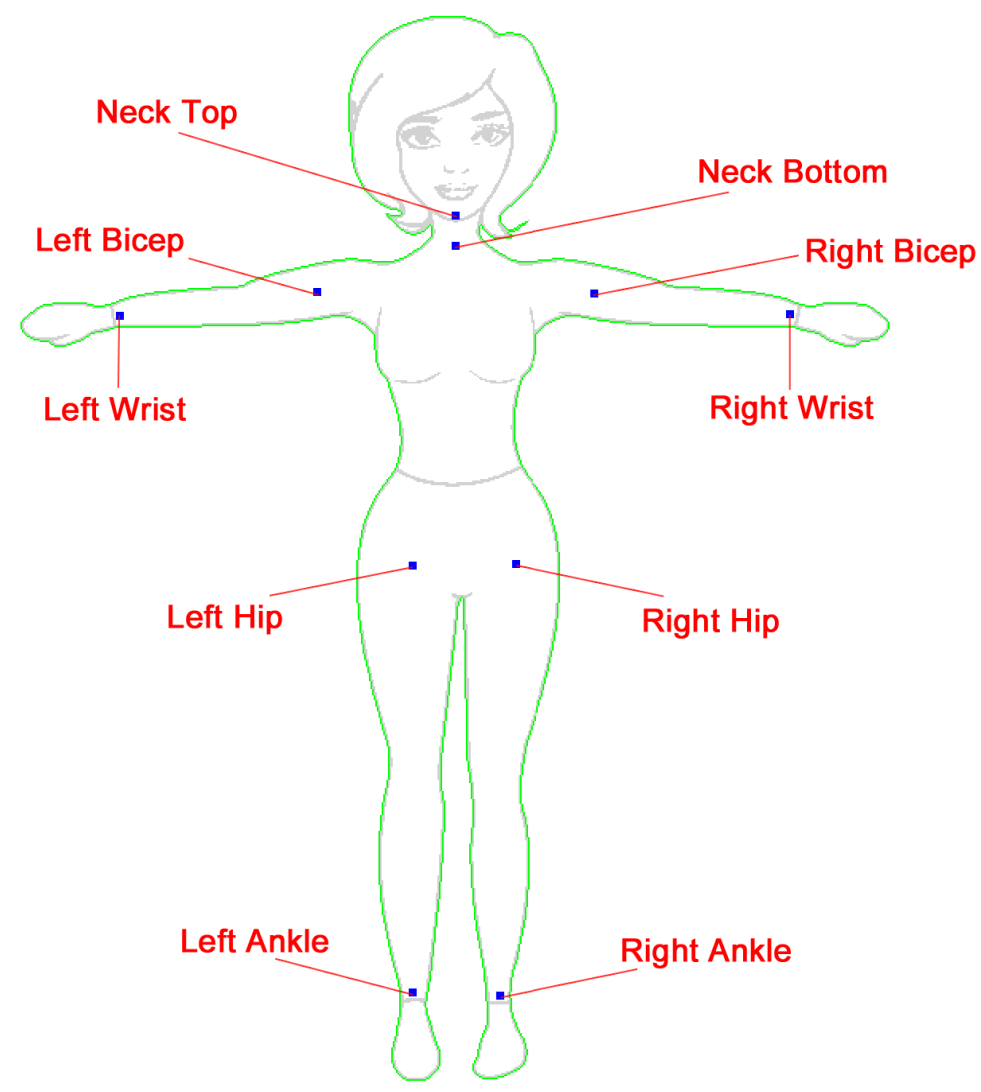

Figure 3.6: Labeled Input Points (User Perspective)

\subsection{D Character Processing}

After input processing, the system performs two 2D processing tasks: adding more skeleton points to the ten existing ones, and mapping between skeleton points and the character outline until the blueprint is complete.

\subsubsection{Terminology}

In this section, we will constantly refer to pixel positions in relation to each other. We use the terms higher and lower to describe the vertical position, of one pixel in relation to another, perceived by the user. Based on the conventional coordinate system, a 
pixel that is higher than another pixel has a smaller y value and, oppositely, a lower pixel has a bigger y value.

In addition, to describe the traced outline, we will interchangeably use the terms "outline" and "skin".

\subsubsection{Arms}

The system starts the process by connecting the bicep and wrist points on each arm with points on the skin. To do that, it applies the following steps for each arm:

- Extend a straight line, which we will refer to as the arm axis, from the bicep point to the wrist point.

- Extend two perpendicular lines from each point, in both directions; i.e. 90 degrees both $\mathrm{CCW}$ and clockwise $(\mathrm{CW})$.

- Find the point of intersection of each perpendicular line with the skin.

- Center the bicep and wrist points between their intersection points.

Figure 3.7 shows the first skin-skeleton mapping process of the character. We refer to the line, formed by two perpendicular lines extended from the point and bounded by two intersection points, by the section line. We use this term to refer to all skin-skeleton mapping lines derived in this methodology section. 


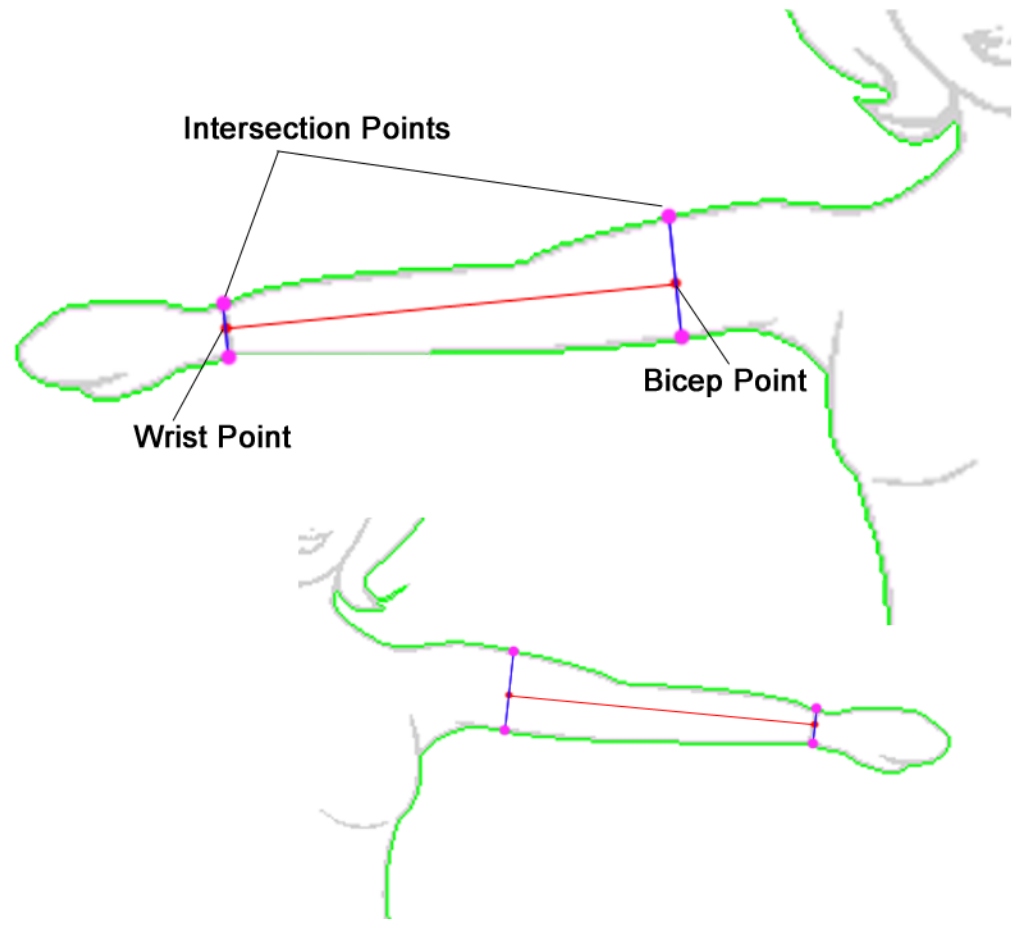

Figure 3.7: Skin-skeleton mapping of arm points. The system extends a line (red) from the bicep to the wrist, forming the arm axis. It then extends lines (blue) perpendicular to the arm axis, towards the outline, and looks for the intersection points (purple) with the skin. Finally, bicep and wrist points are centered between the two purple intersection points.

\subsubsection{The Intersection Algorithm}

We implemented the point-line distance algorithm in our system to find intersection points. Our system uses this algorithm because it always guarantees finding an intersection when two sets of pixels cross each other. The other approach of finding an intersection is to search for mutual or common pixels between the two sets. However, based on our experience, that approach failed to recognize multiple expected intersections. Figure 3.8 shows an intersection example that is recognized by the pointline distance algorithm but not recognized by the "mutual pixel" approach. 


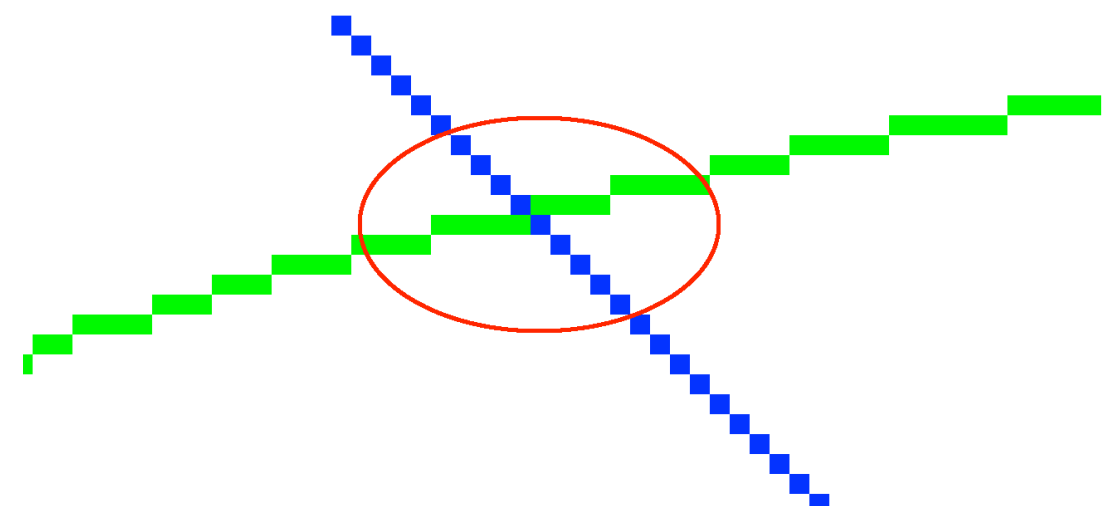

Figure 3.8: An intersection between point set 1 (green) and point set 2 (blue). As illustrated (circled), there are no mutual pixels between the two sets even though they intersect. In this case, a point-line distance algorithm always works

\subsubsection{Arm Sectioning}

To help the system catch further details from the outline, and produce a more refined mesh, we add more skin-skeleton mappings in each body part, but we keep the amount of mappings within a certain threshold so that the resulting mesh remains simple and basic.

To add skin-skeleton mappings to the arm, the system divides the arm axis into $s$ equally sized sections, such that their size is at least equal to threshold $g$, which is fixed at 20 for the arm. This means that from the bicep line to the wrist line, the system creates s1 equally spaced midpoints on the arm axis. Spacing between midpoints is measured as the 2D Euclidean distance between their pixel coordinates.

After that, similar to bicep and wrist points, the system extends two lines perpendicular to the arm axis from each midpoint, towards the outline, to find the intersection points, and then centers each midpoint. Figure 3.9 shows the arms after sectioning their axes, and centering the midpoints. Notice how the arm axis is no longer a 
straight line after the adjustment of midpoints. This is the final look of the arm section lines before it is converted to $3 \mathrm{D}$.

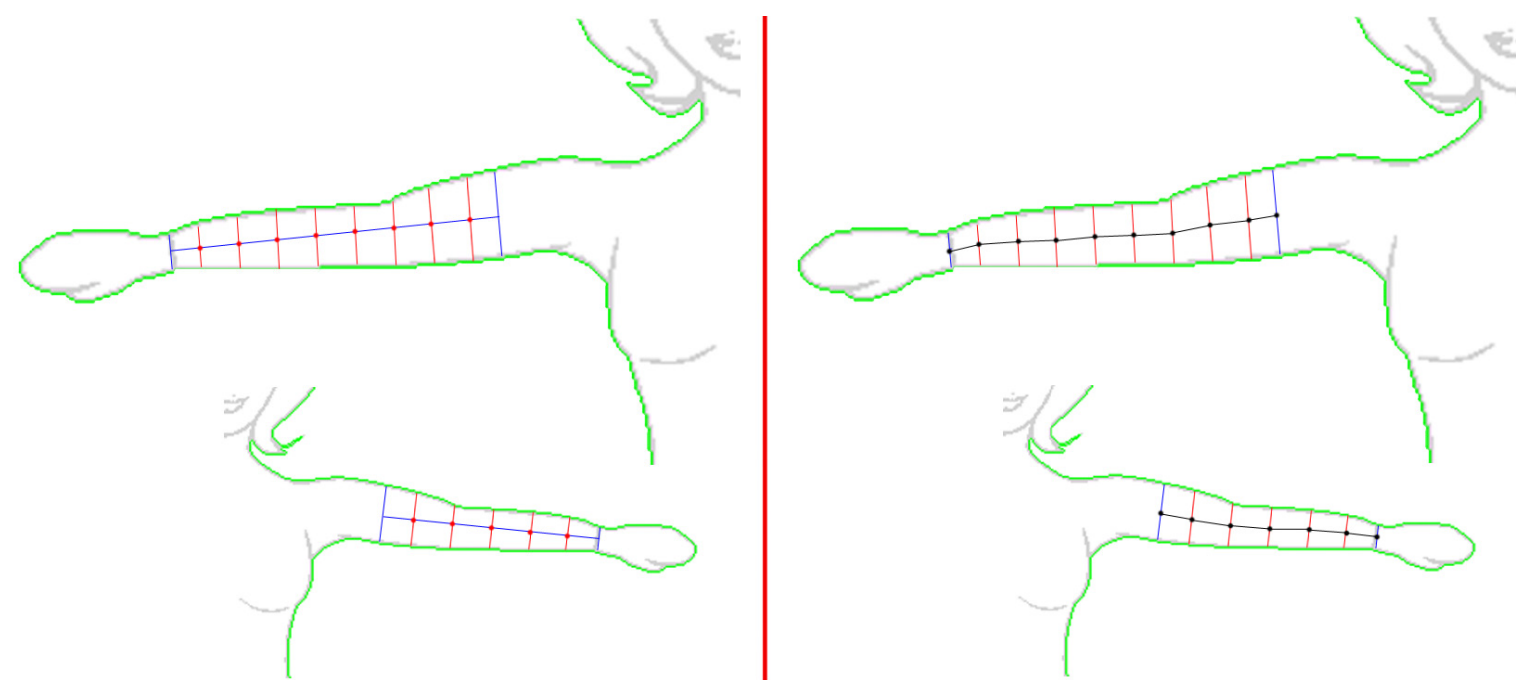

Figure 3.9: Arm Sectioning. Equally sized sections of the arm axis (left). Perpendicular lines are extended from the midpoints to associate them with intersecting points on the outline. Then, midpoints are centered (right) between their intersection points. The arm axes (black) are no longer straight lines

\subsubsection{Legs}

Processing the legs is somewhat different from processing the arms because the hip point location to the leg is not equivalent to the bicep point location to the arm. First of all, the system creates a leg axis, which is the line extended from the hip point to the ankle point. Then, instead of extending perpendicular lines, the system extends horizontal lines from the ankle point on both sides. The reason why we chose the ankle lines to be horizontal is that, in general, the actual axis of the leg tends to be somewhat vertical especially below the knees, and having horizontal lines extended from the ankles is better, compared to lines perpendicular to the axis. 
After that, the system calculates the coordinates of the top middle point between the legs, which we will refer to as the crotch point. The motivation behind this step is clarified later in this subsection. To calculate the location of the crotch point, the system uses the inner leg curve that starts and ends at the following points:

- Start: The right intersection point of the horizontal line extended from the ankle point of the left leg.

- End: The left intersection point of the horizontal line extended from the ankle point of the right leg.

Figure 3.10 shows the leg axes, ankle horizontal lines, and the curve used by the system to get the crotch point.
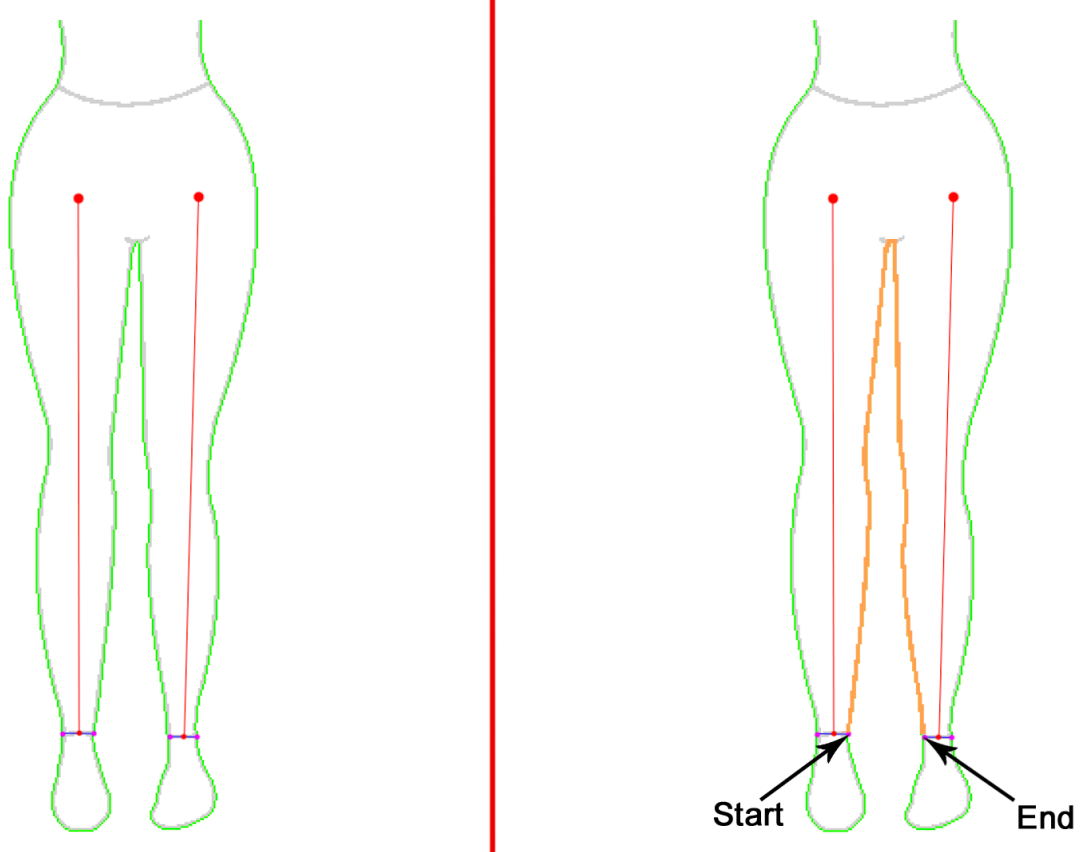

Figure 3.10: The leg axes (red), horizontal ankle lines (blue), and intersection points (purple). In the right picture, the inner leg curve (in orange) is used to retrieve the crotch point. Note the start and the end of the curve 
To locate the crotch point, the system finds the highest pixel on the inner leg curve. However, if the tip of the curve is wide and contains more than one minimum point, then the crotch point is the median tip pixel. The crotch point is important because the system needs it to locate, what we refer to as, pelvis corners. Pelvis corners are two points, one on the left and the other on the right side of the crotch point, on the inner leg curve that meet the following conditions:

- When applicable, they are the horizontally furthest pixels from the crotch point on the curve

- They are lower than the crotch point by 1 pixel

Figure 3.11 shows the resulting crotch point and pelvis corners.

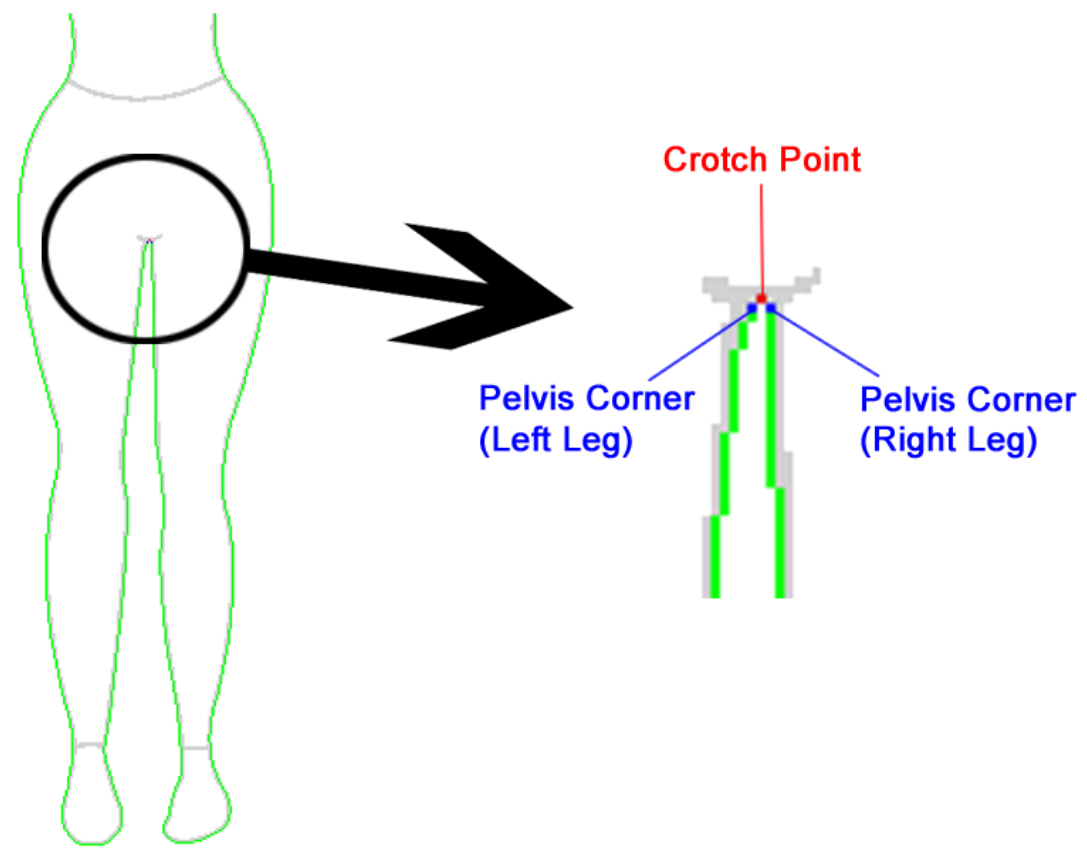

Figure 3.11: Crotch Point \& Pelvis Corners. Top middle point (red), referred to as the crotch point, is used to derive the pelvis corners (blue), which are a pixel lower than the crotch point. 


\subsubsection{Leg Sectioning}

After finding the pelvis corners, the system uses them to create the thigh line on each leg, which starts at the leg's pelvis corner, and extends towards the outer side of the leg, ending at the intersection point. While the section lines of the arm are all perpendicular to the arm axis, sectioning the leg starts with the thigh line, that is perpendicular to the leg axis, gradually rotating the next lines until, approximately half way through the axis, the rest of the lines become horizontal. Figure 3.12 shows the leg section lines, which is the final look of the leg section lines before it is converted to 3D.
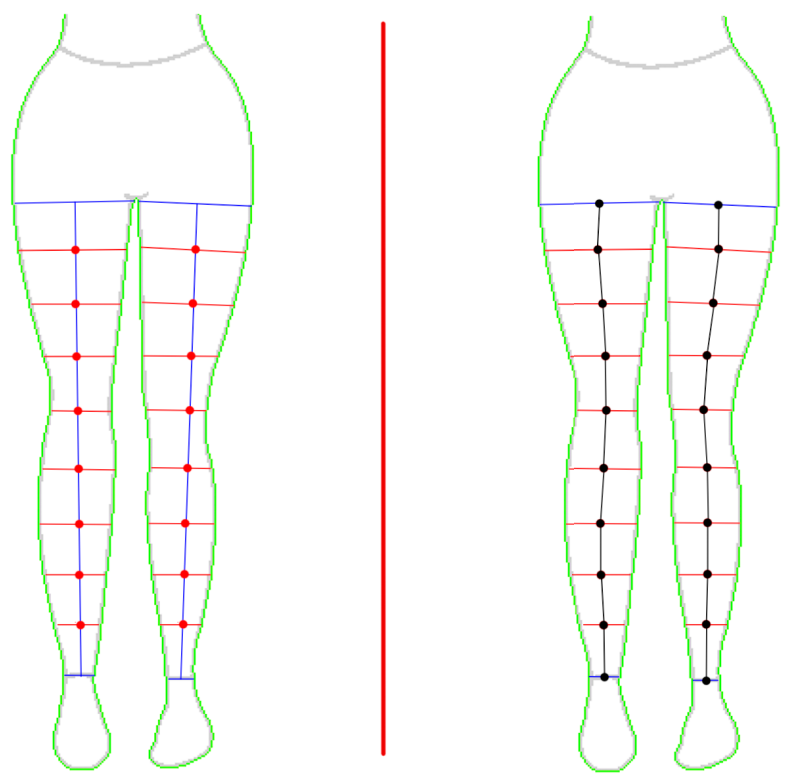

Figure 3.12: Leg Sectioning. Equally sized sections of the leg axis (left). Lines are extended from the midpoints to associate them with intersecting points on the outline. The top (thigh) line is perpendicular to the axis while the rest is gradually rotated until eventually horizontal. Finally, midpoints (right) are centered.

This process is similar to arm sectioning, in terms of calculating equally spaced midpoints on the axis, and centering midpoints between intersection points. The 
difference is that not all leg lines are perpendicular to the leg axis, and the section size threshold for legs is fixed at 25 instead of 20.

\subsubsection{Limb Axis Correction}

There are cases when the initial limb axis represented as a straight line does not entirely fit in the corresponding limb. This occurs when the area bounded by the limb outline is very small, or if the limb is not straightly extended. As a result, our algorithm automatically adjusts the axis when it fails to find an intersection point for every perpendicular line extended from the axis midpoints. Figure 3.13 demonstrates the automatic axis correction process.

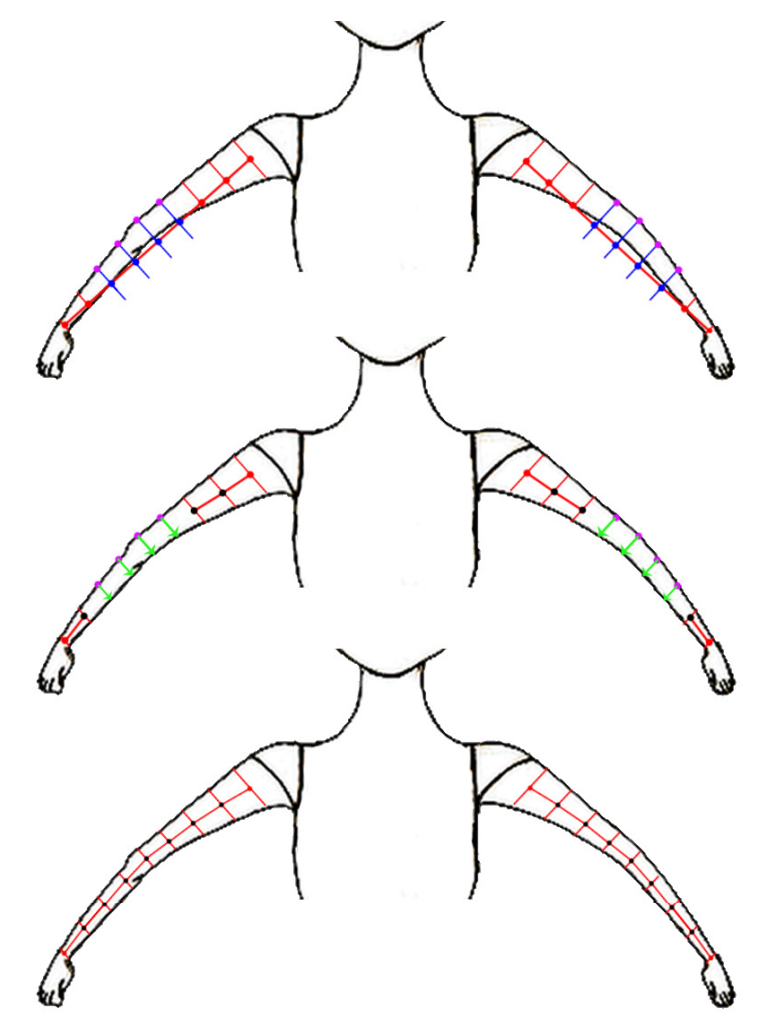

Figure 3.13: Limb Axis Correction Process. Top: some midpoints (blue) have intersection points (purple) from one side only because of unfitted axes. Middle: using single-sided intersections, the system extends perpendicular lines (green) towards the opposite side to find the second intersection. Bottom: midpoints are centered 


\subsubsection{Head}

After sectioning the arms and legs, the system starts processing the character's head. To the system, the head is formed in the outline by the pixels that are higher than the neck top point (Figure 3.6). Because the head outline may involve both head and hair detail, we created an algorithm that tries to estimate the size of the head excluding the hair volume. To do that, we slightly rely on realistic head measurements to calculate the width and height of the head [58]. The final dimensions are not 100\% realistic but based on empirical tests with the system, we found that they produce a head size that approximately excludes the character's hair (see Appendix B for mesh results).

That being said, we would like to clarify that excluding hair from the character mesh is an approach we decided to follow in our algorithm, which assumes that the modeling artist would create the hair as a separate 3D surface and then attach it to the character mesh. A second approach that modelers may use is to construct the hair strands of the character with the same mesh, by subdividing the surface of the character's head. While the second approach is still doable with the mesh that our system produces, determining how the character's hair is created is beyond the scope of our algorithm.

\subsubsection{Neck Sectioning}

To prepare for the head sectioning process, the system first finds intersections between the two neck points and the outline, using horizontal lines (Figure 3.14), and then centers the neck points between their intersection points. 


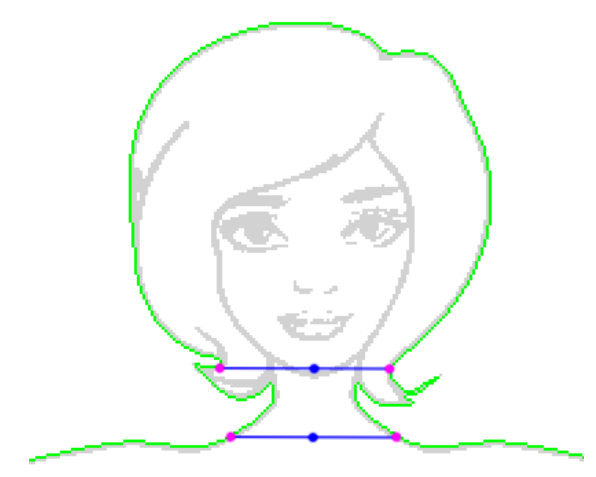

\section{Figure 3.14: The neck points and their horizontal intersections (purple) with the outline}

After forming the intersection lines of the neck top and bottom, the system divides the neck area into two equally sized sections, adding a middle point between the neck top and bottom. Next, it determines the neck width. Because user input can vary from user to user, and because portions of the neck outline may involve garment detail like a collar for example, our algorithm calculates the neck width by vertically traversing the neck section from top to bottom, to retrieve the smallest horizontal diameter in that section. To calculate the smallest diameter, the system starts from the y value of the neck top, travels down the neck by incrementing the y value 1 pixel at a time, calculating the horizontal outline intersections at each y value. The diameter of the intersection line is the distance between the left intersection point and right intersection point.

After reaching the y value of the neck bottom, calculating all possible diameters, the system assigns the value of the smallest diameter to the neck width, which is assigned to both the neck middle and bottom lines. Assigning the width to those two lines means that the distance between the left intersection point and the right intersection point no longer defines their diameters. Their diameters are instead fixed at the neck width value. 
On the other hand, final width of the neck top is determined by the head measurements that we will explain next in this section.

Figure 3.15 shows the smallest diameter found in the neck section, the neck middle with its width-constrained horizontal line, and the neck bottom with the adjusted width-constrained line. If the original bottom line's width is already equal to the smallest diameter, then the system does not change it. It only then creates the middle line with that same width.

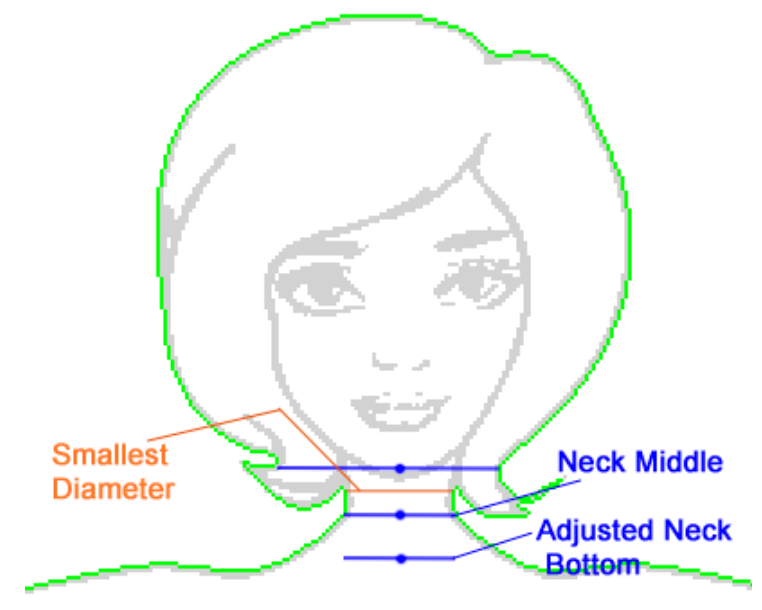

Figure 3.15: Neck Sectioning. The smallest diameter (orange) found between the neck top and bottom, the neck middle and its neck-width line, and the neck bottom with its neck-width adjusted line

\subsubsection{Estimating Head Dimensions}

To determine the width and height of the head, the system applies two different approaches to calculate those dimensions, and then chooses the best approach. The first approach starts by calculating the height $h_{l}$ from the head outline, then deriving the head width $w_{1}$ from $h_{1}$. The second approach is calculating the width $w_{2}$ from the head outline, and deriving the height $h_{2}$ from that width. The system uses the values of the first 
approach by default, unless $w_{2}$ is smaller than $w_{1}$. We chose to always use the smaller width and the height associated with it because, based on empirical evidence, it proved to be a more consistent approach in approximately excluding hair volume from the character.

To calculate $h_{1}$ and $w_{2}$, the system first locates the head tip. The head tip is found by extending a vertical line $l$ from the centered neck top point, up towards the top of the head outline. The intersection point between $l$ and the outline is the head tip, and the length of $l$, is $h_{l}$. After that, the system horizontally sections the head, dividing $l$ into three equally sized sections. In the middle section, the system finds the smallest diameter of the outline of that section, and assigns its value to $w_{2}$. Figure 3.16 shows a summary of calculated dimensions of the head.

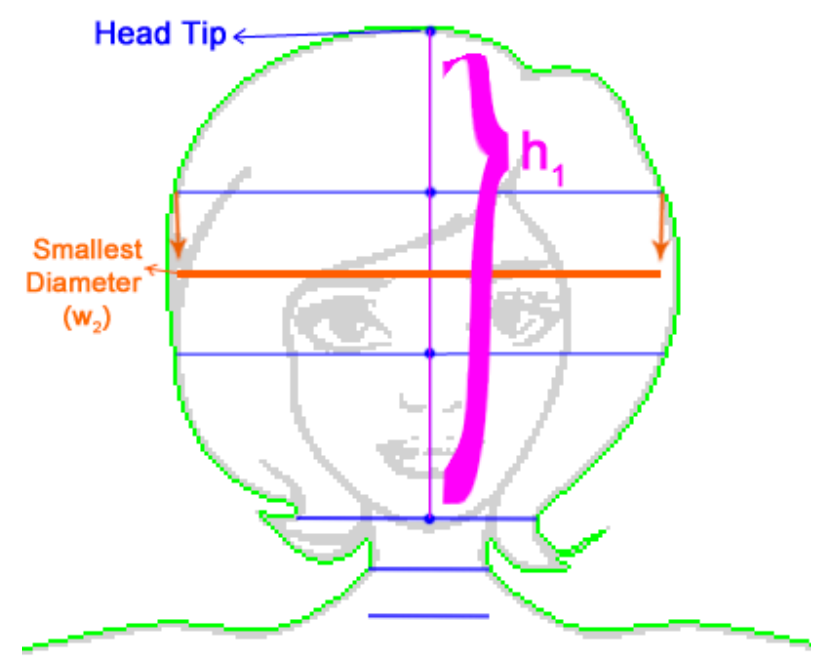

Figure 3.16: A summary of calculated dimensions of the head. For the first approach of dimension calculation, height $h_{l}$ is the length of $l$, the line between the head tip and the neck top point. For the second approach, width $w_{2}$ is the smallest diameter found in the middle section of the head 
After calculating $h_{1}$ and $w_{2}, w_{1}$ and $h_{2}$ are derived in the first approach and the second approach, respectively, as the following:

$$
\begin{aligned}
& w_{1}=2 / 3 h_{1} \\
& h_{2}=3 / 2 w_{2}
\end{aligned}
$$

Having calculated dimensions of each approach, the system uses values of the second approach if and only if $w_{2}$ is smaller than $w_{1}$. In addition, if the second approach is used, a new head tip is calculated and a new vertical line $l$ is formed, using the neck top as the starting point, to match the new height of the head.

\subsubsection{Head Sectioning}

After determining the final head dimensions, the system sections the head using eight lines (Figure 3.17), with different widths and positions proportionate to the width and height of the head, respectively. Table 3.1 displays the dimensions of each head section line, and the adjusted width of the neck top line. As mentioned earlier, these ratios are based on relatively standard proportions [58], and are empirically adjusted. 


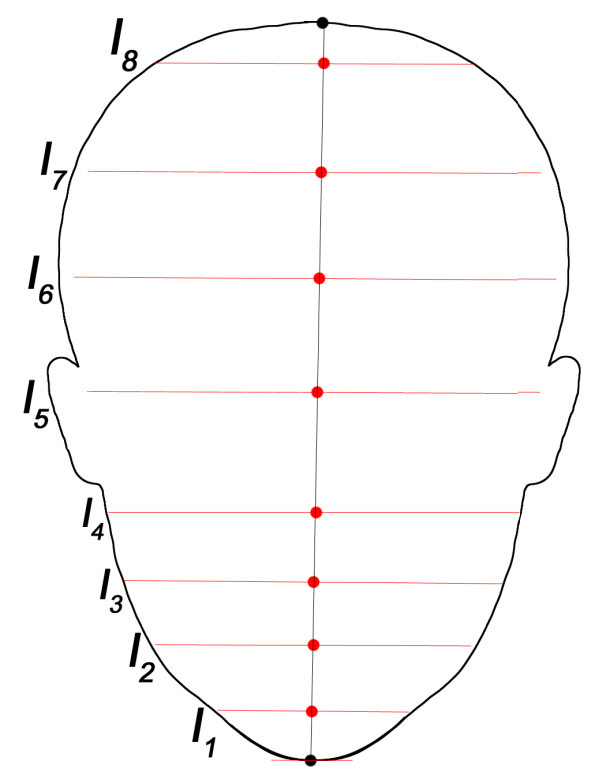

Figure 3.17: Eight section lines vertically dividing the head

Table 3.1: Width and Height ratios given for each head section line (Figure 3.17)

\begin{tabular}{|c|c|c|}
\hline & Width & Height \\
\hline $\begin{array}{l}\text { Neck top line } \\
\text { (head bottom) }\end{array}$ & $1 / 4 W$ & 0 (starting point) \\
\hline $\mathbf{L}_{1}$ & $3 / 4 W$ & $7 / 96 H$ \\
\hline $\mathbf{L}_{2}$ & $13 / 16 W$ & $7 / 48 H$ \\
\hline $\mathbf{L}_{3}$ & $7 / 8 W$ & 7/32 H \\
\hline $\mathbf{L}_{4}$ & $15 / 16 W$ & $7 / 24 H$ \\
\hline $\mathbf{L}_{5}$ & $W$ & $7 / 16 H$ \\
\hline $\mathbf{L}_{6}$ & $35 / 32 W$ & $7 / 12 H$ \\
\hline $\mathbf{L}_{7}$ & $25 / 24 W$ & $35 / 48 H$ \\
\hline \multirow[t]{2}{*}{$\mathrm{L}_{8}$ (head top) } & $15 / 16 W$ & $7 / 8 H$ \\
\hline & $W=$ head width & $H=$ head height \\
\hline
\end{tabular}


Unlike other body parts, head sectioning does not depend on intersection lines with the outline. Instead, the algorithm relies more on standard head proportions, and instead of having outline-skeleton mappings, we have skeleton points, the midpoints between the head tip and the neck top point, with fixed-width lines whose ends serve as skin points.

\subsubsection{Neck Width Revisited}

The system re-evaluates the neck width to check if it is greater than the width of the head. As mentioned earlier, this can happen if there is garment or hair detail that is contributing to the size of the outline in the neck area, and resulting in what appears to be a neck that is greater than the head in width. To control that, if the width of the neck is greater or equal to the head's, the system changes it to be equal to the width of the neck top line, which is only one quarter the width of the head (Table 3.1). This new width is assigned to both the neck middle and bottom lines.

\subsubsection{Torso}

\subsubsection{Chest Line}

For the torso, the system starts by finding what we refer to as the chest line. The chest line is the line whose ends are points that define the character's underarms. To find the underarm points, we use an automatic body feature extraction algorithm [57] to detect the underarm curve. The way our implementation of the algorithm works is: starting from the inner intersection point of the bicep line in each arm, and traveling its way through the outline towards the character's torso, the underarm point is the first point whose pixel 
direction changes 90 degrees or more than the starting point. Figure 3.18 shows the detected underarm points, and the formed chest line.

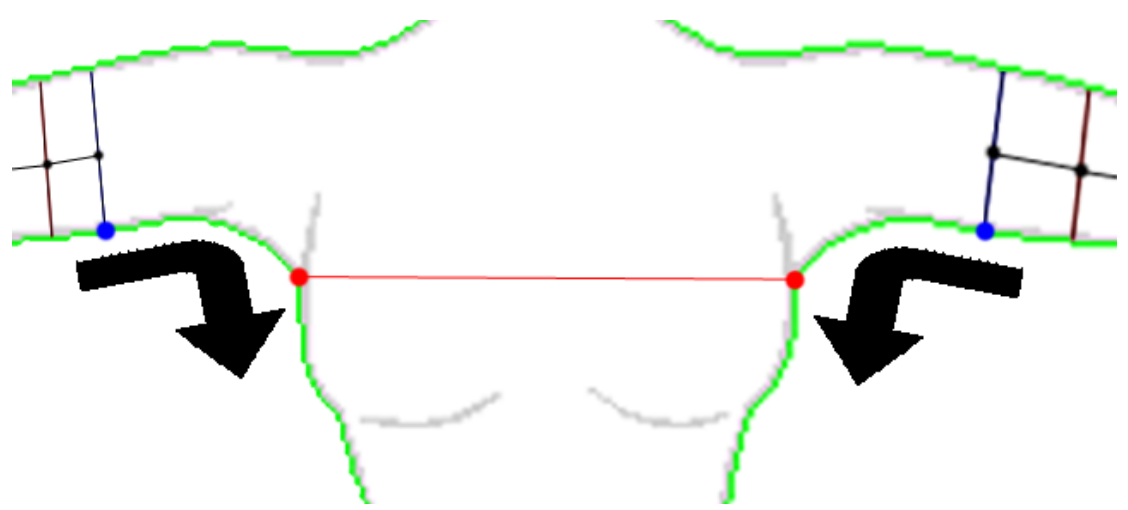

Figure 3.18: Underarm \& Chest Line detection. The algorithm starts from the inner intersection points (blue) of the bicep lines, until it reaches a pixel (red) whose direction changes 90 degrees or more than the starting point, forming the chest line

In some cases, the two underarm points may have different y values; thus, one of them is higher than the other. To keep the torso section lines horizontal, the system adjusts the position of the higher underarm point to be on the same y level as the lower underarm point. To achieve that, the system continues traveling along the outline until it finds the pixel with a bigger y value, which matches the other underarm point.

\subsubsection{Clavicle Line}

The second torso line the system forms is referred to as the clavicle line. This line is aimed to estimate the position of the character's clavicle or collarbone, and it is important in the process of connecting the torso with the arms that we will explain in the next section. There are two methods of forming the clavicle line. The default method is used when the system succeeds in finding intersection points with the outline and forms the clavicle line accordingly. The alternative method is when the system fails to find the 
suitable intersection points, for reasons that we will explain, and thus forms the clavicle line using an alternative algorithm.

In the first method, the system extends a vertical line from each underarm point up towards the outline to find an intersection point. After finding the two intersection points, the system evaluates the portion of the outline that is bordered by the clavicle intersection points and the neck bottom intersection points (Figure 3.14). To explain how the system finds the clavicle line ends, we will refer to the left neck bottom, right neck bottom, left clavicle, and right clavicle intersection points as $n_{0}, n_{1}, c_{0}$, and $c_{l}$, respectively. The system also calculates $m$, the midpoint between $c_{0}$ and $c_{1}$.

The system determines the final clavicle points as follows: it first finds the lowest pixel or pixels on the outline portion between $n_{0}$ and $c_{0}$. It does the same with the outline portion between $n_{l}$ and $c_{l}$. If multiple pixels are found on a side, it picks the closest pixel to $m$. Finally, to ensure that the clavicle line is horizontal, the system evaluates the y value of the chosen pixel on each side. If the two y values are not equal, it adjusts the lower point to match the y value of the higher point, by moving through the same portion of the outline again, to find a pixel, closest to $m$, that matches the y value of the higher pixel. Figure 3.19 shows how the clavicle line is found using the first method. 


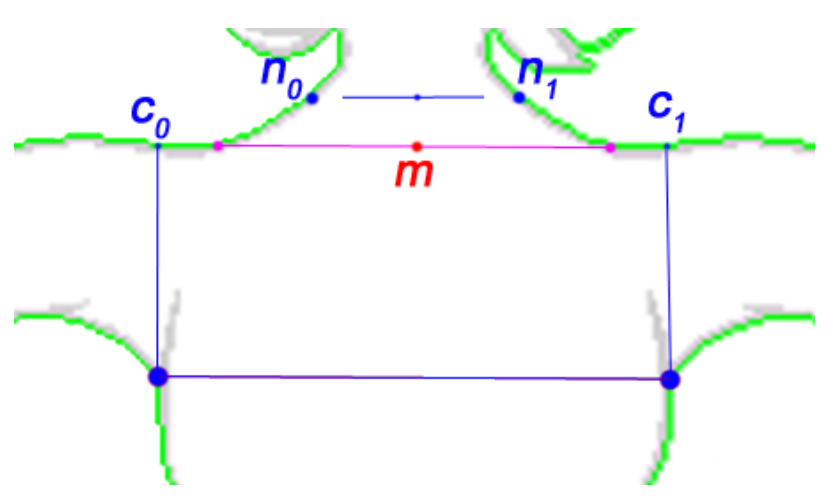

Figure 3.19: The first method of forming the clavicle line. The system searches for the lowest pixels in the outline portion (green) between $c_{0}$ and $n_{0}$, and between $c_{1}$ and $n_{1}$. The clavicle points (purple) are the lowest, and, closest to $m$, pixels forming the final clavicle line (purple)

There are two cases when the system needs to use an alternative approach to form the clavicle line. The first case is when $m$ is at the same y level as the neck bottom point, which means that $c_{0}, n_{0}, c_{1}$ and $n_{l}$ are on the same y level. Consequently, the clavicle line is formed on the neck bottom line, which poses a problem when the character is converted to 3D. The second case is when the system finds intersection points, between the vertical lines extended from the underarm points and the outline, that are higher than the neck bottom points, in which case the clavicle line will be above the neck area. These cases of faulty intersections can appear due to nonrealistic character proportions, unpredictable user input, or an outline that involves garment or hair detail around the neck and shoulder of the character.

To solve these cases, the system uses the alternative approach. It forms a clavicle line that has the same width as the neck bottom line. Then it calculates its vertical distance from the neck bottom line as follows:

Calculate the vertical distance $d$ between the neck bottom line and the chest line, and then compare two values $y_{0}$ and $y_{1}$ where 


$$
y_{0}=d * 0.1
$$

and

$$
y_{1}=3
$$

If $y_{0}$ is greater than $y_{1}$, place the clavicle line $y_{0}$ pixels below the neck bottom line. Else, place it $y_{l}$ pixels below.

\subsubsection{Shoulder Line}

In order for the system to combine the arms with the torso and have enough geometry when it transforms the character into a 3D mesh, our algorithm adds an additional line between the clavicle and the chest lines, referred to as the shoulder line. The shoulder line is exactly in the middle between the clavicle and chest lines, and its width is the average width of the two lines. Figure 3.20 shows the formed shoulder line. It is the last line the system adds in the top area of the torso. The rest of the torso lines are formed below the chest line to define the lower torso area.

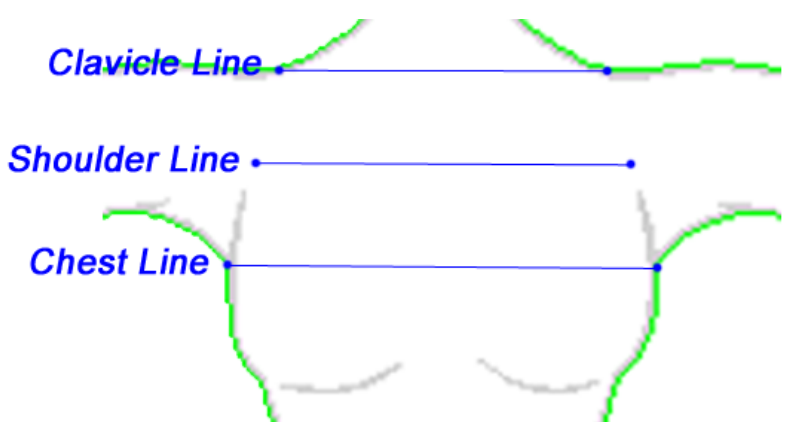

Figure 3.20: The Shoulder Line. It lies between the chest and clavicle lines, and its width is the average of their widths

\subsubsection{Lower Torso}

For the rest of the torso, the system inserts five equally spaced horizontal lines between the chest line and thigh lines (Figure 3.12). To do that, the system first calculates 
$m_{0}$, the midpoint of the chest line and $m_{1}$, the midpoint of the thigh lines, which is the mid distance between the left intersection point of the left thigh line, and the right intersection point of the right thigh line. After that, the system divides the distance between $m_{0}$ and $m_{1}$ using five equally spaced midpoints. Finally, it extends two horizontal lines from each midpoint, towards the character outline, one line to the left, and the other to the right of the midpoint, forming five section lines in the lower half of the torso (Figure 3.21).

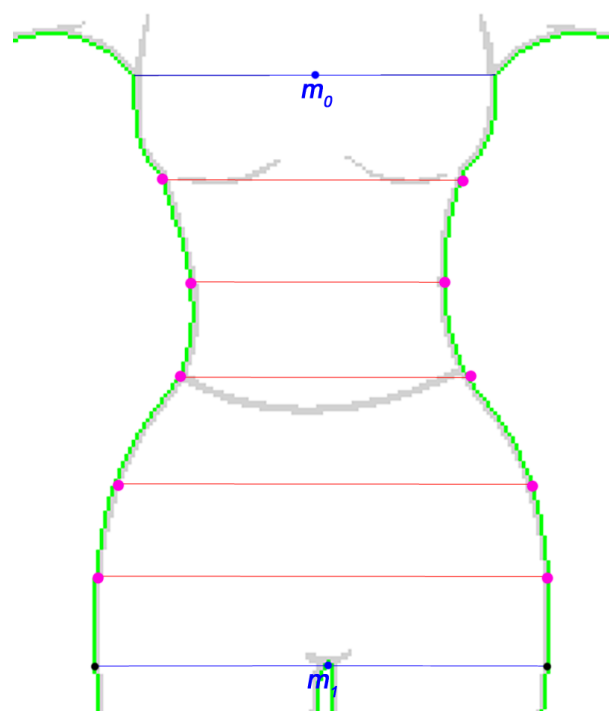

Figure 3.21: Five torso lines formed between the chest and thigh lines

\subsubsection{Shoulders}

The last part the system needs to process before 3D construction is shoulders. The system forms the area of each shoulder with three lines placed between the upper torso and the arm. Figure 3.22 shows the three shoulder lines. The first line is extended between the clavicle line end and the chest line end of the same side, crossing the shoulder line end in the middle. The other two lines are extended between two portions of the character outline. The first portion, $p_{0}$, is the one that starts at the clavicle line end, 
ending at the outer intersection point of the bicep line. The second portion, $p_{l}$, is the one that starts at the chest line end and ends at the inner intersection point of the bicep line.

Resuming line forming in the shoulder area, the second line is extended between points $a_{0}$ and $a_{1}$ in $p_{0}$ and $p_{1}$, respectively, such that the distance between $a_{0}$ and the clavicle line end is $33 \%$ the length of $p_{0}$, and the distance between $a_{l}$ and the chest line end is $33 \%$ the length of $p_{1}$. The third line is extended between points $b_{0}$ and $b_{1}$ in $p_{0}$ and $p_{1}$, respectively, such that the distance between $b_{0}$ and the clavicle line end is $66 \%$ the length of $p_{0}$, and the distance between $b_{1}$ and the chest line end is $66 \%$ the length of $p_{1}$.

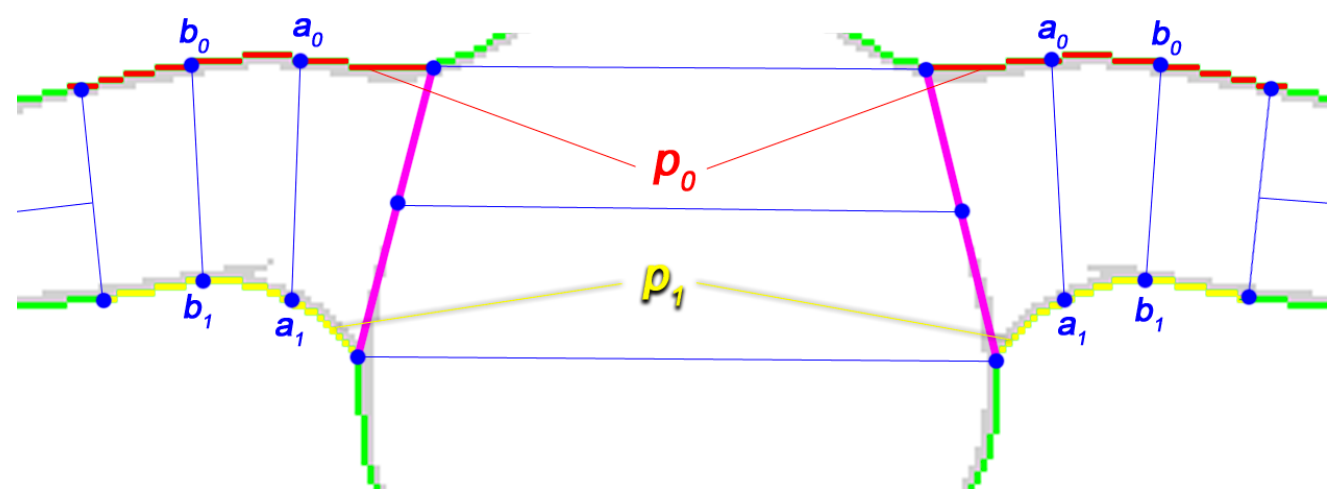

Figure 3.22: Three shoulder lines. The first (purple) starts at the clavicle line end and ends at the chest line end. The second is bounded by $a_{0}$ and $a_{1}$, and the third is bounded by $b_{0}$ and $b_{1}$

Adding the shoulder lines is the last step in $2 \mathrm{D}$ processing that the system applies before 3D construction. Before we move to the next section, Figure 3.23 presents the final 2D blueprint of all the section lines that the system automatically derives using the 10 user-input points. 


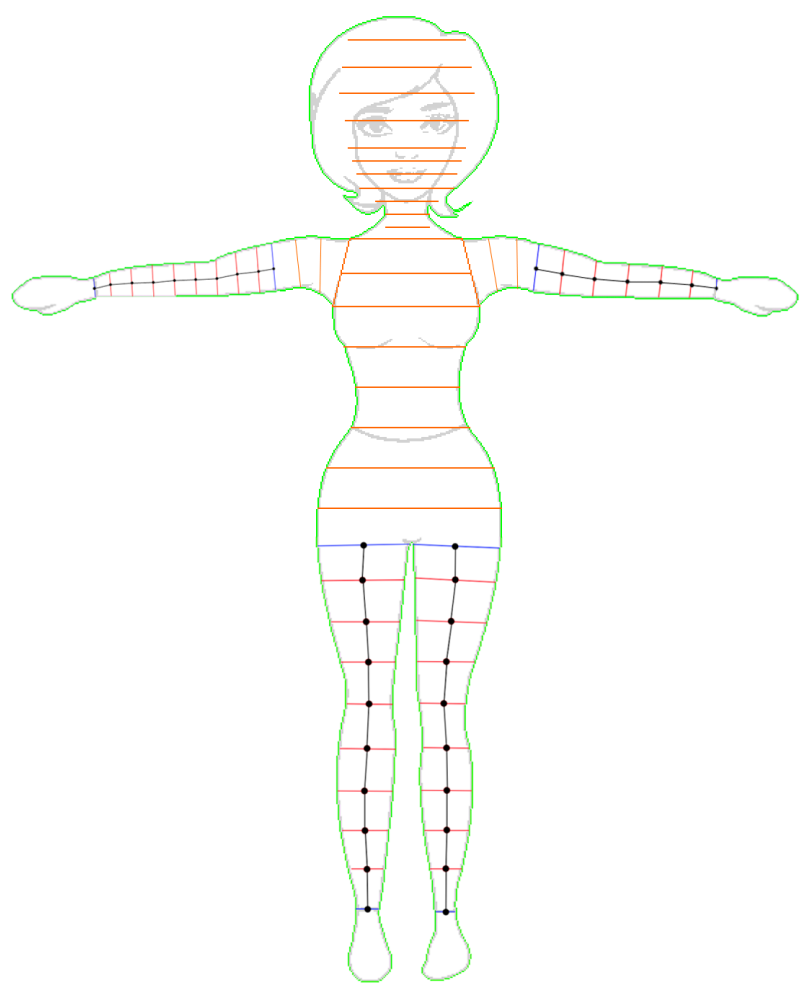

Figure 3.23: A complete 2D blueprint of the sketched character

\subsection{D Construction}

The $3 \mathrm{D}$ construction process is divided into depth estimation, mesh construction and mesh smoothing. In the first subsection, we introduce our depth estimation algorithm for each body part, explaining our approach in combining a few standard body proportions with a method to allow the flexibility of creating nonstandard depth proportions.

Next, in Subsection 3.8.2, after we briefly discuss the process of automatically creating 3D profile curves and converting them into polygon surfaces using Maya commands, we explain in detail our approach to stitch the character's body parts in accordance with the recommended topology. We also explain our adjustment and 
smoothing approach to automatically fix anomalies that may have resulted from the body combining process, and to smooth joined areas.

\subsubsection{Depth Data}

As Lopez et al. [59] state, one of the drawbacks of automatic 3D construction from a single image is the ambiguity in inferring depth information. Because our system only focuses on human characters, we have the advantage of implementing some depth constraints that are only suitable for a human character structure. Similar to 2D head estimation explained in Subsection 3.7.5, we lightly rely on standard human proportions to estimate depths of the head and torso, while combining some rules we created to flexibly create nonrealistic body proportions like characters with tiny arms and big heads, for example. In general, we avoid applying standard global constraints that force specific depth ratios between body parts.

The system controls the depth of the character using its $2 \mathrm{D}$ blueprint. This means that the system provides section lines with depth (z-axis or third-dimension) information, which ultimately transforms the blueprint into a 3D structure. In this subsection, we explain how depths of body parts are calculated, and in subsection 3.8.2, we demonstrate the results when the $3 \mathrm{D}$ mesh is constructed.

\subsubsection{Limbs \& Neck}

For both arms and legs, our algorithm keeps the 3D structure in its simplest form by making the depth of each limb section line, including thigh lines, equal to its width, creating circular arms and legs. This decision is influenced by the box modeling process, where limbs are blocked out using cylinders [60]. In addition, for simplicity reasons, the 
algorithm keeps the neck middle and bottom lines circular, but locally manipulates them during the smoothing process described in Subsection 3.8.2.

\subsubsection{Head}

Similar to the approach we used to estimate the head's width and height in Subsection 3.7.5, we derive its depth $d$ using relatively standard proportions [58], which we adjusted based on empirical tests with a variety of character sketches. $d$ is calculated as follows:

$$
d=7 / 8 h
$$

where $h$ is the height of the head.

Figure 3.24 displays the 8 head section lines, as well as the neck top line, in front and side views, and Table 3.2 shows our final head depth ratios that the system uses for the section lines. In addition, it shows the adjusted depth of the neck top line.

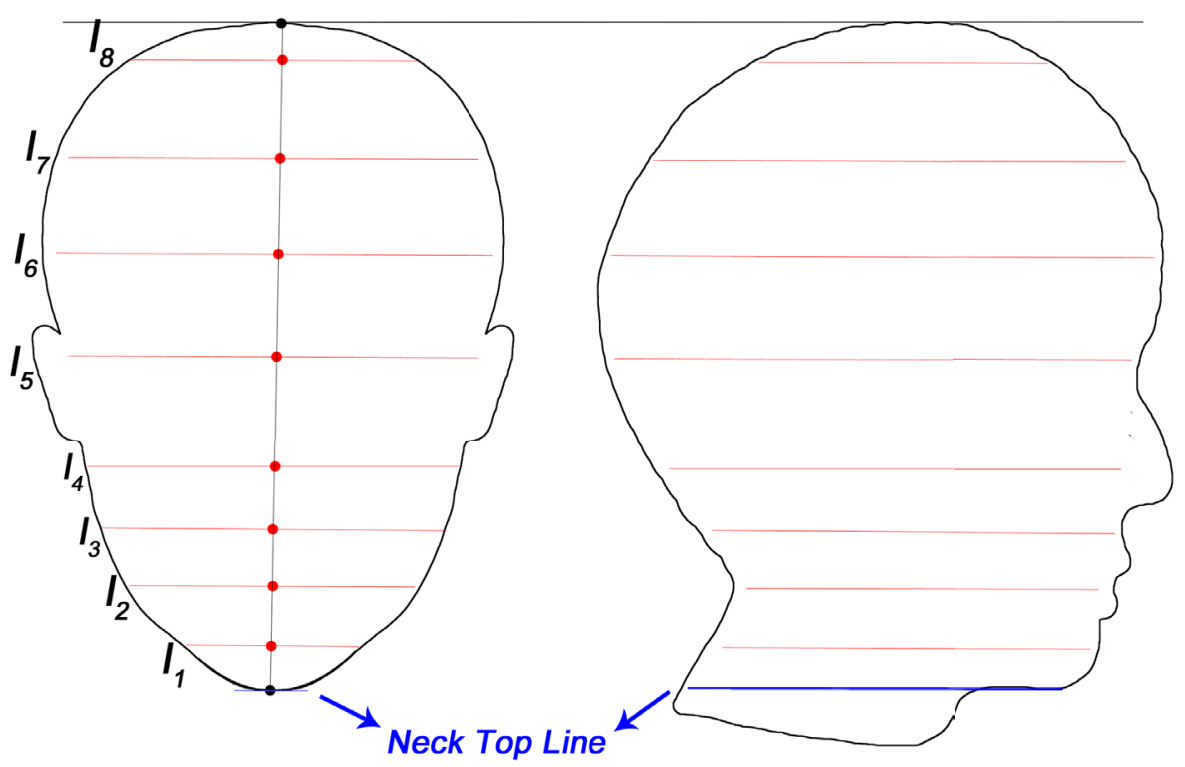

Figure 3.24: Head section lines (red) and neck top line (blue) in front and side views 
Table 3.2: Depth ratios given for each head section line (Figure 3.24)

\begin{tabular}{cc}
\hline & Depth \\
\hline $\begin{array}{c}\text { Neck top line } \\
\text { (head bottom) }\end{array}$ & $5 / 7 d$ \\
\hline $\mathrm{L}_{1}$ & $31 / 42 d$ \\
\hline $\mathrm{L}_{2}$ & $21.98 / 28 d$ \\
\hline $\mathrm{L}_{3}$ & $11.52 / 14 d$ \\
\hline $\mathrm{L}_{4}$ & $6 / 7 d$ \\
\hline $\mathrm{L}_{5}$ & $20 / 21 d$ \\
\hline $\mathrm{L}_{6}$ & $17 / 18 d$ \\
\hline $\mathrm{L}_{7}$ & $13 / 14 d$ \\
\hline $\mathrm{L}_{8}($ head top) & $11 / 14 d$ \\
\hline \hline
\end{tabular}

\subsubsection{Chest Line}

Estimating the chest depth $d_{c}$ is an important step because the depths of both upper and lower torso section lines are proportionate to it. Calculating $d_{c}$ is based on empirical tests to estimate a suitable depth for the character's torso, and is influenced by depth measurements of a human body, retrieved from NASA's anthropometric data [61], in relation to the arms and neck. In addition, $d_{c}$ is constrained by width $w_{c}$ of the chest line. $d_{c}$ is first assigned the greater value among the two following expressions:

$$
d_{n} * 1.05
$$




$$
d_{b}+d_{r}
$$

where $d_{n}$, in (7), is the depth of the neck bottom, which is equal to its width as mentioned earlier. In (8), $d_{b}$ and $d_{r}$ are the depths of the bicep lines of the left and right arms, respectively. If $d_{c}$ is greater than $w_{c}$, then it is adjusted to be:

$$
d_{c}=w_{c} / 2 * 1.45
$$

\subsubsection{Upper Torso}

There are two lines in the upper torso between the neck bottom line and the chest line: the clavicle and shoulder lines. The system calculates the depth $d_{l}$ of each line based on the quadratic Bezier function:

$$
d_{l}=(1-t)^{2} p_{0}+2(1-t) t p_{1}+t^{2} p_{2}
$$

such that

$$
t=\operatorname{line}_{y} / y_{\text {dist }}
$$

where

$$
\begin{aligned}
& \text { line }_{y}=m_{l}-m_{n} \\
& y_{\text {dist }}=m_{c}-m_{n}
\end{aligned}
$$

In equation $10, p_{0}$ is the bottom neck depth, $p_{2}$ is the chest depth, and $p_{1}=p_{2}-1$. In equation $12, m_{l}$ is the midpoint of the upper torso line in process and $m_{n}$ is the midpoint of the neck bottom line. Finally, in equation $13, m_{c}$ is the midpoint of the chest line.

\subsubsection{Lower Torso}

To estimate the lower torso depth, the system first calculates the hip depth $d_{h}$, which is equal to the average depth of the thigh lines. In addition, it retrieves $m_{1}$, the midpoint of the thigh lines calculated in Subsection 3.7.6. After that, for each lower torso 
line, the system calculates the depth $d_{l}$ by linearly interpolating between $d_{c}$ and $d_{h}$ as follows:

$$
d_{l}=(r * \text { diff })+d_{c}
$$

such that

$$
\begin{aligned}
& r=\text { line }_{y} / y_{\text {dist }} \\
& \text { diff }=d_{h}-d_{c}
\end{aligned}
$$

where

$$
\begin{aligned}
& \text { line }_{y}=m_{l}-m_{c} \\
& y_{\text {dist }}=m_{1}-m_{c}
\end{aligned}
$$

In equation $17, m_{l}$ is the midpoint of the lower torso line in process, and $m_{c}$, used in equations 17 and 18, is the midpoint of the chest line.

\subsubsection{Torso Alignment}

A simple torso alignment process is applied after depths are calculated. The system aligns all torso depth lines, by moving them along the z-axis, so that the back of the character is in a straight line when viewed from the side. The alignment starts from bottom to top keeping the lowest torso depth line $d_{t}$ unchanged, while adjusting the rest of the torso depth lines, as well as, the neck bottom depth line, accordingly. The reason why we keep $d_{t}$ unchanged is to facilitate the process of combining the torso with the legs. Thus, similar to leg depth lines, we leave $d_{t}$ in the center of the z-axis. This simple process is reflected in the $3 \mathrm{D}$ mesh constructed in the next subsection. 


\subsubsection{Mesh Construction \& Smoothing}

In this subsection, we discuss the $3 \mathrm{D}$ construction of the character using $2 \mathrm{D}$ information of body section lines as well as their calculated depths. We also explain our approach to adjust and smooth the mesh in different body parts. In this part of the process, the algorithm involves a number of Maya commands used to automatically build the character. However, to keep the information in this thesis relevant to audience without prior Maya knowledge, we avoid discussing specific tools or commands, and try to be as general and clear as possible when it comes to Maya-specific discussions.

\subsubsection{Polygon Surfaces}

To create the character's polygon mesh, the system needs to convert 2D and depth data into a 3D structure that define the shape of the character. To do that, the system uses the relative position and orientation of each body section line and converts it into a 3D profile curve that reflects the depth of the section line in the z-axis. Reflecting the depth on those profile curves means that a side view of the character is finally constructed, from depth and alignment data. Since the limbs and neck section lines are in their simplest forms, their corresponding profile curves are 3D circles.

Using profile curves, the system constructs polygon surfaces that are defined and fittingly shaped by those curves. In addition, each profile curve forms an edge loop on the surface with the same position and orientation. Figure 3.25 shows front and side views of the polygon surfaces. 


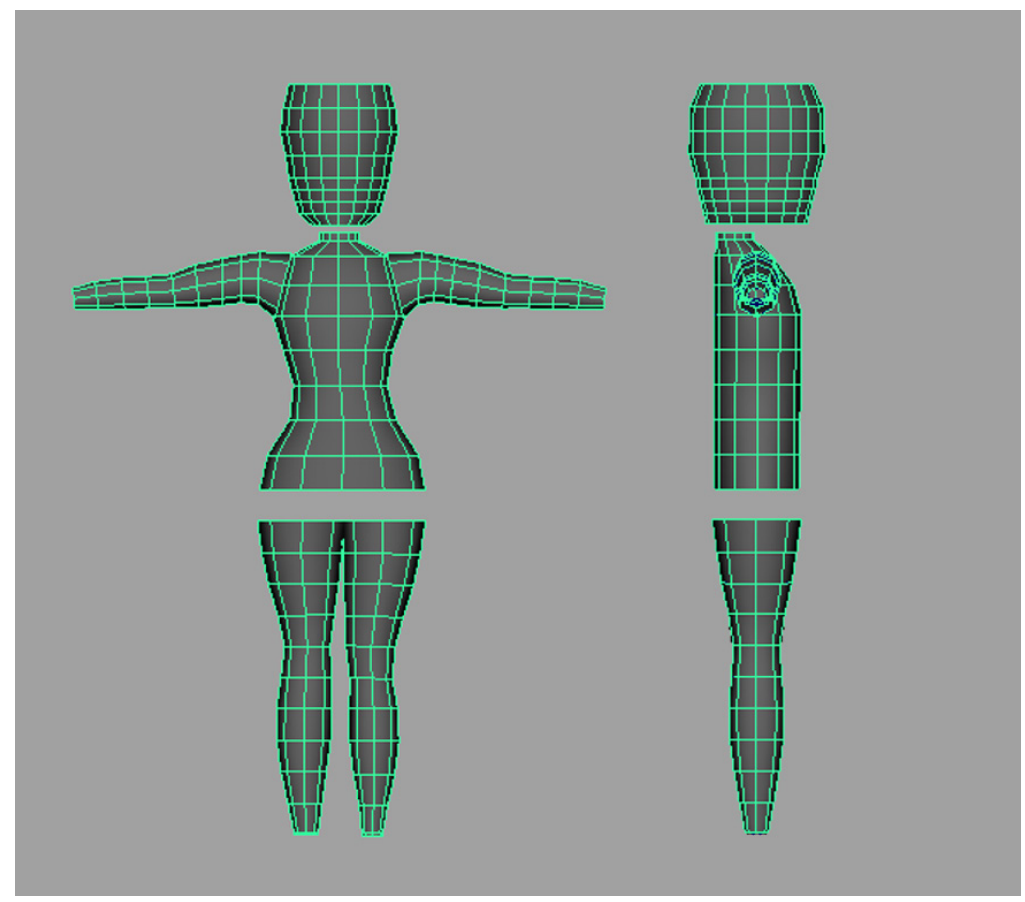

Figure 3.25: Polygon surfaces constructed using 2D coordinates and orientation, as well as depth data

\subsubsection{Maya's Polygon API}

During the final phase of the project that includes connecting mesh parts and locally adjusting the character model, we intensively studied how Maya implements its polygons' data structure, in order to be able to automatically manipulate the mesh. We focused on understanding the index system of mesh components, namely faces, edges and vertices. The main goal was to ensure that our system consistently selected and manipulated the targeted mesh component.

That being said, we will not discuss further details about how internal data structures work in Maya and how we implemented them in our system. We will instead focus on the tasks that the system automatically accomplishes using its comprehensive knowledge about the internal structure of polygon meshes and Maya commands. 


\subsubsection{Head \& Neck}

To close the top and bottom of the head mesh, the system adds a surface on each end, attaching it to the mesh with top and bottom vertices, respectively. It then subdivides those surfaces into smaller polygons to create a suitable edge flow for the head (Figure $3.26)$.

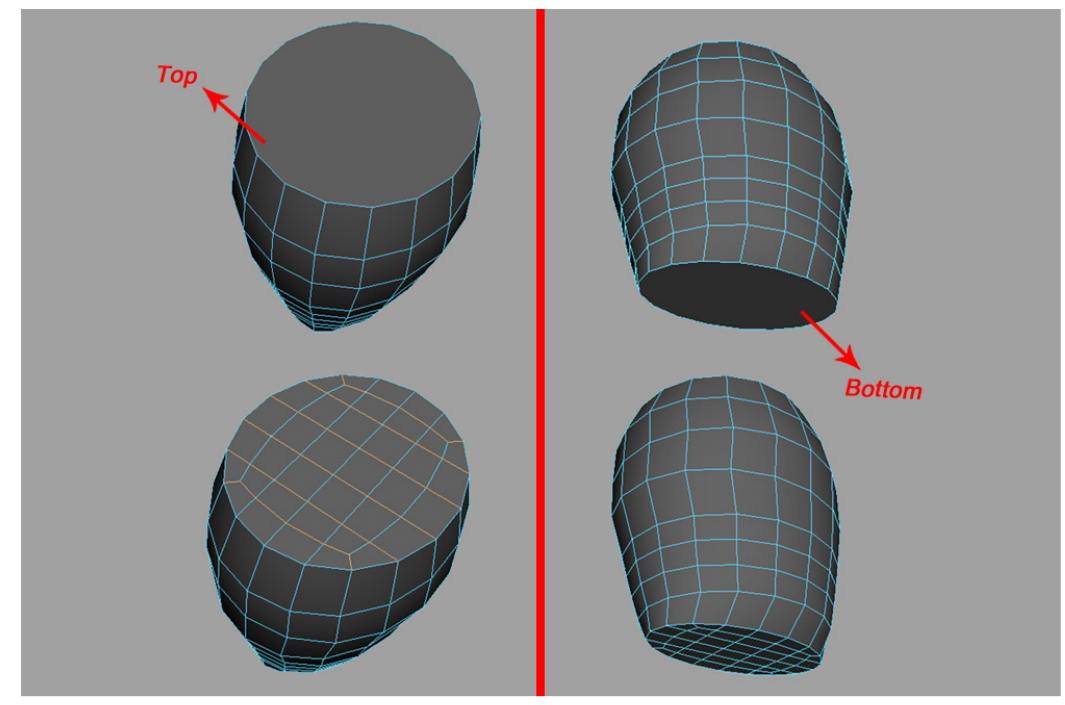

Figure 3.26: The top and bottom surfaces of the head. The first row is before automatic subdivision. The second row is after subdividing with suitable edge flows

After that, the system smoothes the top surface by adjusting the horizontal edge rings (orange lines in Figure 3.26). For each edge ring $e_{i}$, the system uses a quadratic Bezier curve (equation 10) where $p_{0}$ and $p_{2}$ are the left and right ends of $e_{i}$, respectively, and

$$
\begin{gathered}
p_{1}=[c \cdot x, c \cdot y+h, c \cdot z] \\
t=d_{0} / d
\end{gathered}
$$

such that $c$ is the middle vertex in $e_{i}$, and 


$$
h=0.5 d
$$

$d_{0}$ is the distance between $p_{0}$ and the vertex of $e_{i}$ to be adjusted, and $d$ is the distance between $p_{0}$ and $p_{2}$. Figure 3.27 demonstrates the top surface smoothing process. Smoothing the bottom surface is done after the system connects it to the neck. On the other hand, this is the final shape of the top surface.

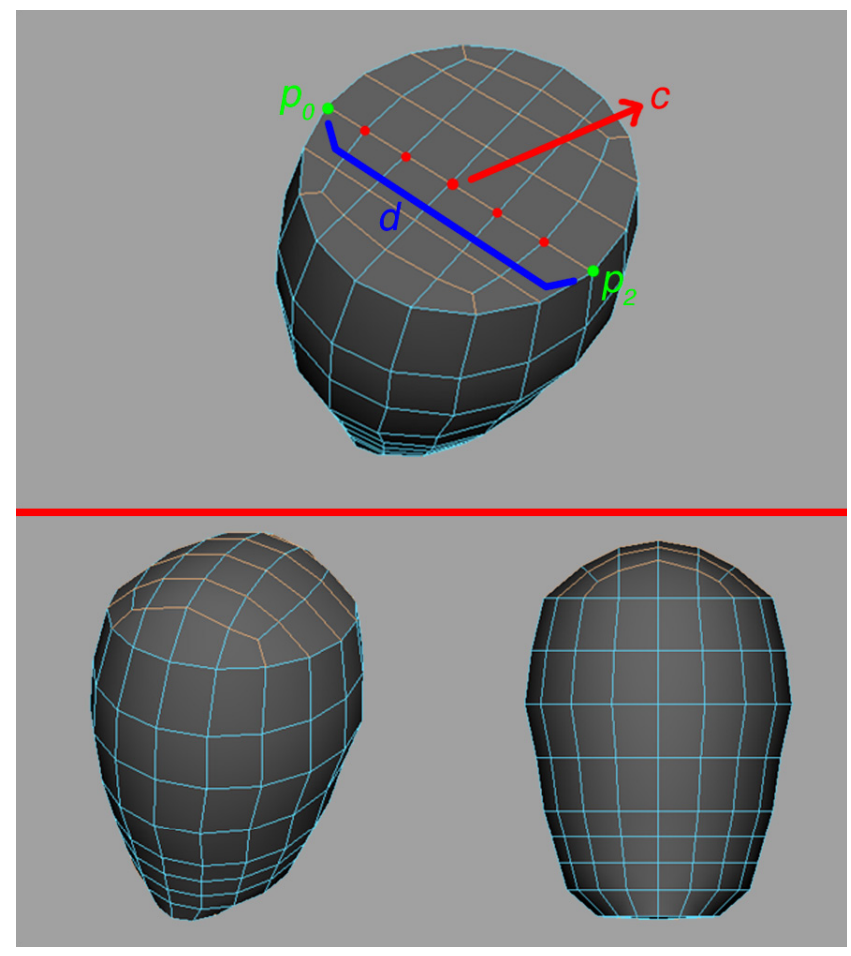

Figure 3.27: Top surface smoothing. Each edge ring (orange) is smoothed with a Bezier curve, adjusting the edge's vertices (red). Bottom image presents the final result

After smoothing the head top, the system attaches the head bottom to the neck by bridging between the neck's top edge loop and the head bottom's edges that surround the back four rows of the surface faces, deleted before bridging. Additional polygons are created between the head and neck (Figure 3.28). 


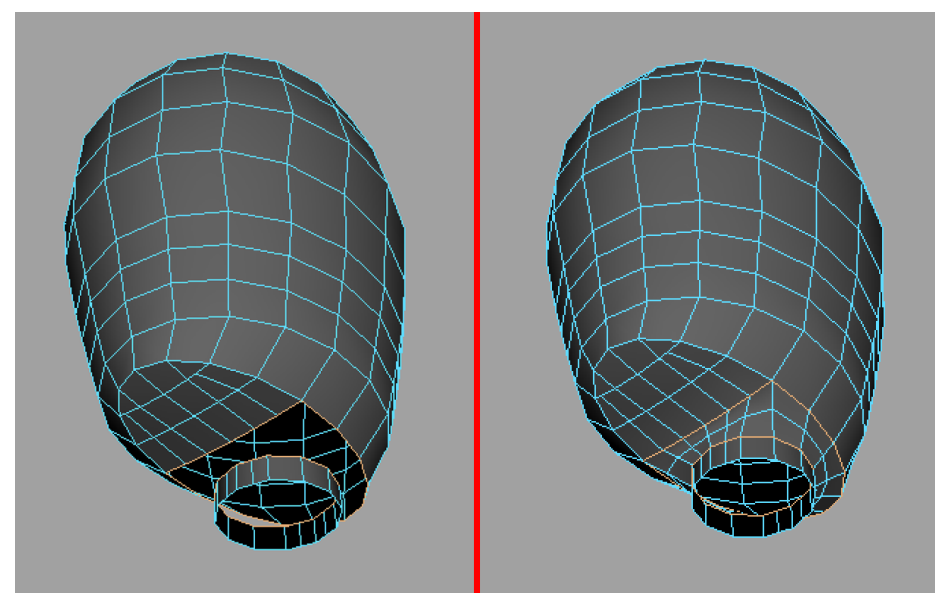

Figure 3.28: Head-Neck Bridging

To smooth the joined head-neck area, the system implements a series of Bezier curves. Figure 3.29 demonstrates them in chronological order. A, B and D apply quadratic curves (equation 10), while $\mathrm{C}$ is the following linear interpolation:

$$
B(t)=(1-t) p_{0}+t p_{1}
$$

As seen in the figure, vertices are color coded to illustrate control points (white, red and green), and the smoothed vertices (yellow and purple). In B and D, initial coordinates of smoothed vertices are used as control points. 


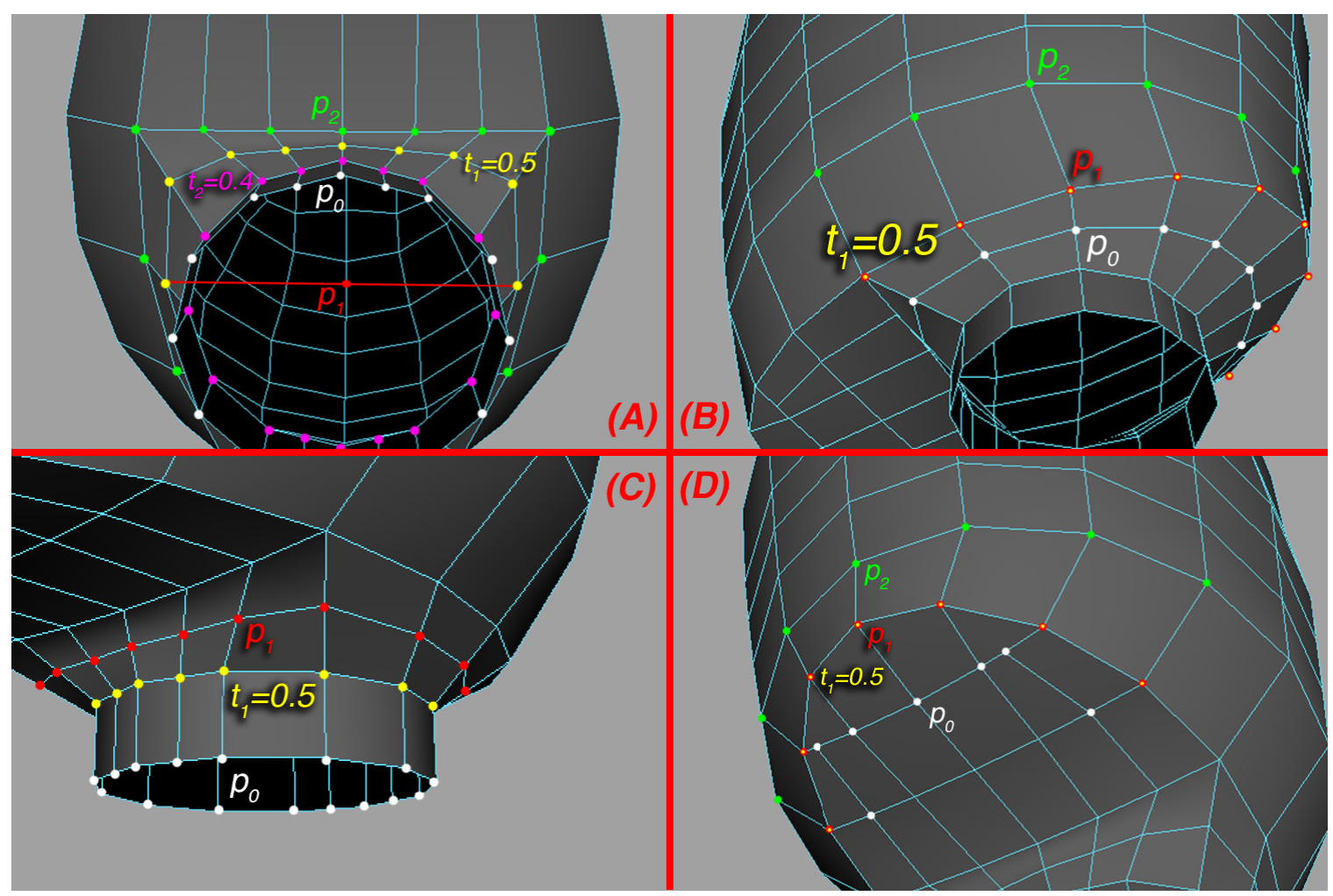

Figure 3.29: Head-Neck Smoothing

In addition, as seen in Figure 3.30, the two corner vertices are adjusted for a smoother look. The new position is the average of the six adjacent vertices. Figure 3.31 shows the mesh before and after smoothing.

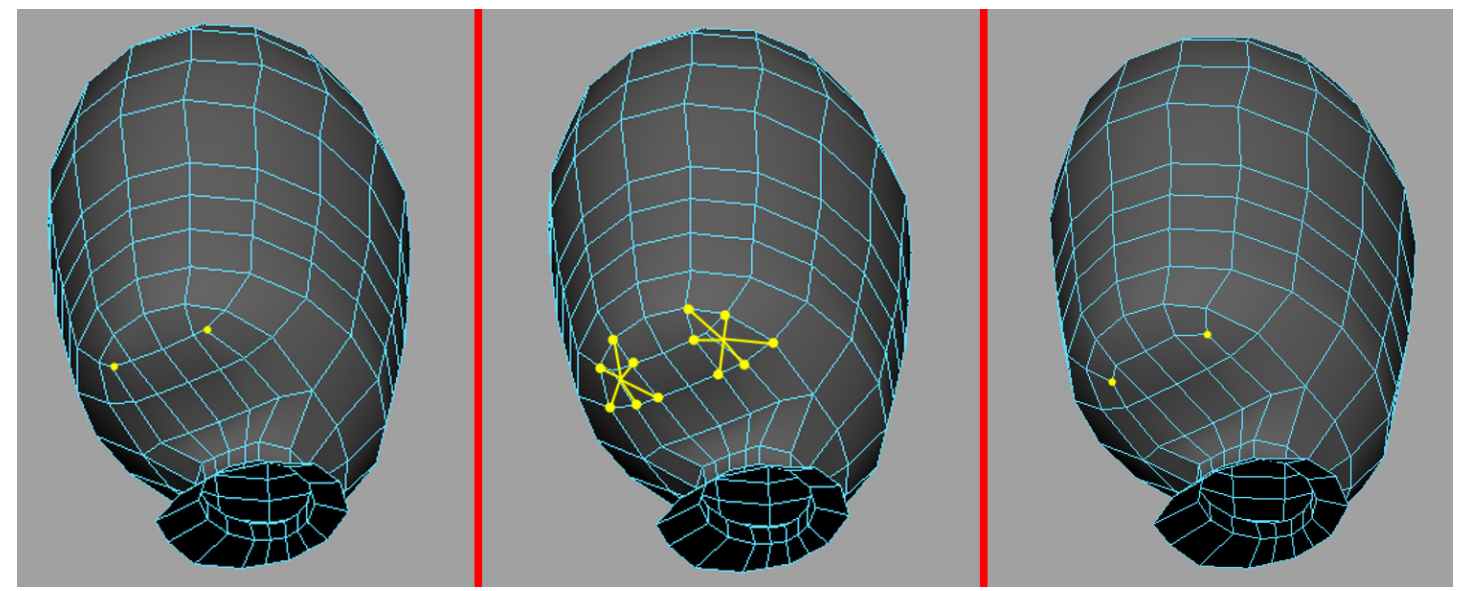

Figure 3.30: Averaging Corner Vertices 


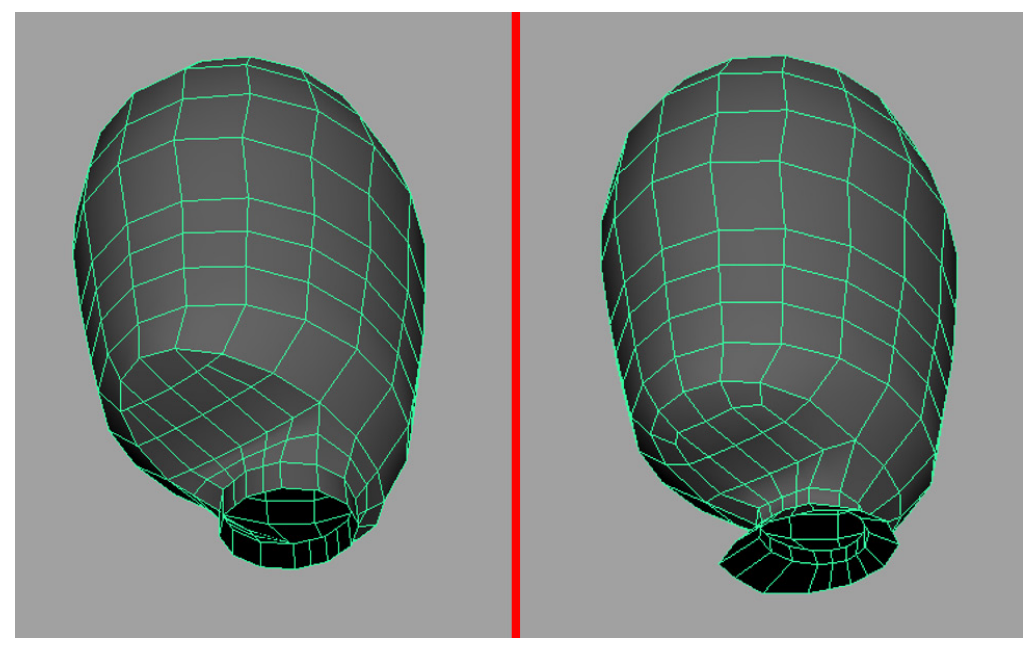

Figure 3.31: Head mesh before (left) and after (right) smoothing

\subsubsection{Torso Depth (Front Adjustment)}

The front of a back-aligned torso is adjusted, below the chest. Adjustments are proportionate to the chest edge loop $e_{c}$. First, the system calculates the width $w_{c}$ of $e_{c}$, which is the distance between the left most vertex $v l_{c}$ and the right most vertex $v r_{c}$ on $e_{c}$. Then, it retrieves $m_{c}$, the midpoint between $v l_{c}$ and $v r_{c}$. Next, for each vertex in the front section of $e_{c}$, the system calculates the distance $d_{c}$ between the vertex and $m_{c}$.

Then, for each torso edge loop $e_{i}$ below the chest, vertices of the front section are adjusted as follows:

- Calculate the width $w_{i}$ of $e_{i}$, the same way $w_{c}$ is calculated for $e_{c}$.

- Calculate the ratio $r_{i}$ of $w_{i}$ to $w_{c}$.

- $\quad$ Retrieve $m_{i}$, the midpoint in $e_{i}$.

- Finally, for each vertex in the front section of $e_{i}$, the distance $d_{i}$ between the vertex and $m_{i}$ is:

$$
d_{i}=r_{i} * d_{c}
$$


Figure 3.32 illustrates the front torso adjustment of one torso edge loop.
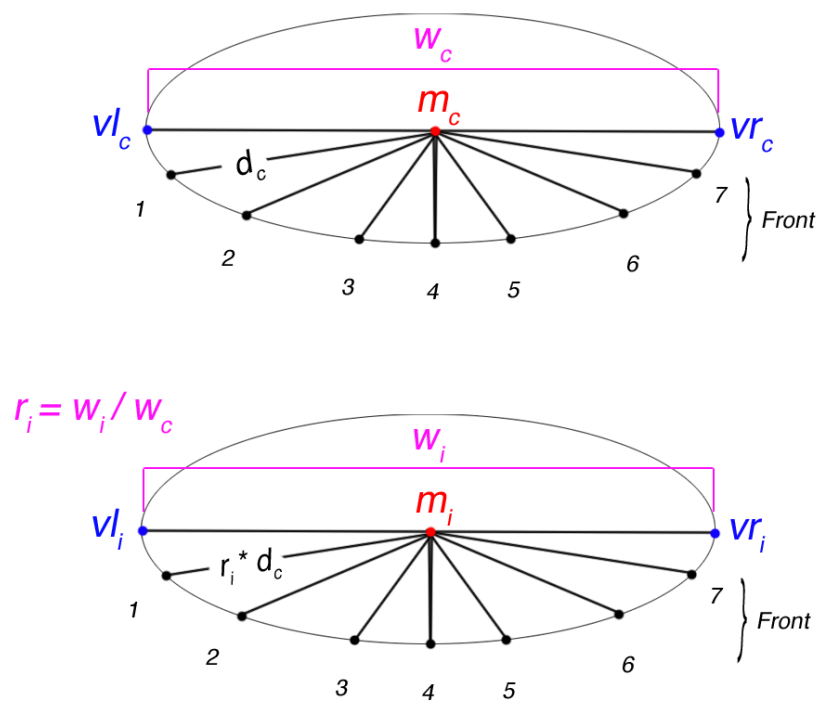

Figure 3.32: Torso Depth Adjustment (illustration in top view). Chest edge loop (top) parameters, and one of the torso edge loops (bottom) proportional adjustment

\subsubsection{Combining Torso \& Legs}

To connect the torso to the legs, the system applies a similar approach to the previous process. It starts by partially bridging between the thigh lines and the bottom edge of the torso, leaving the middle section of the torso unconnected. Before constructing the middle surface, the system checks if an overlap between legs occurs. If there is an overlap, it resolves it by ensuring that the distance between inner leg vertices is at least 0.1 centimeters. This minimal distance gives enough space for the middle surface to be constructed without overlapping geometry (Figure 3.33). After that, the system constructs a single surface that attaches the edges of the inner legs with the middle section of the torso. Then, similar to the head surfaces, it subdivides the surface into smaller polygons, creating a suitable edge flow in the connected area. 


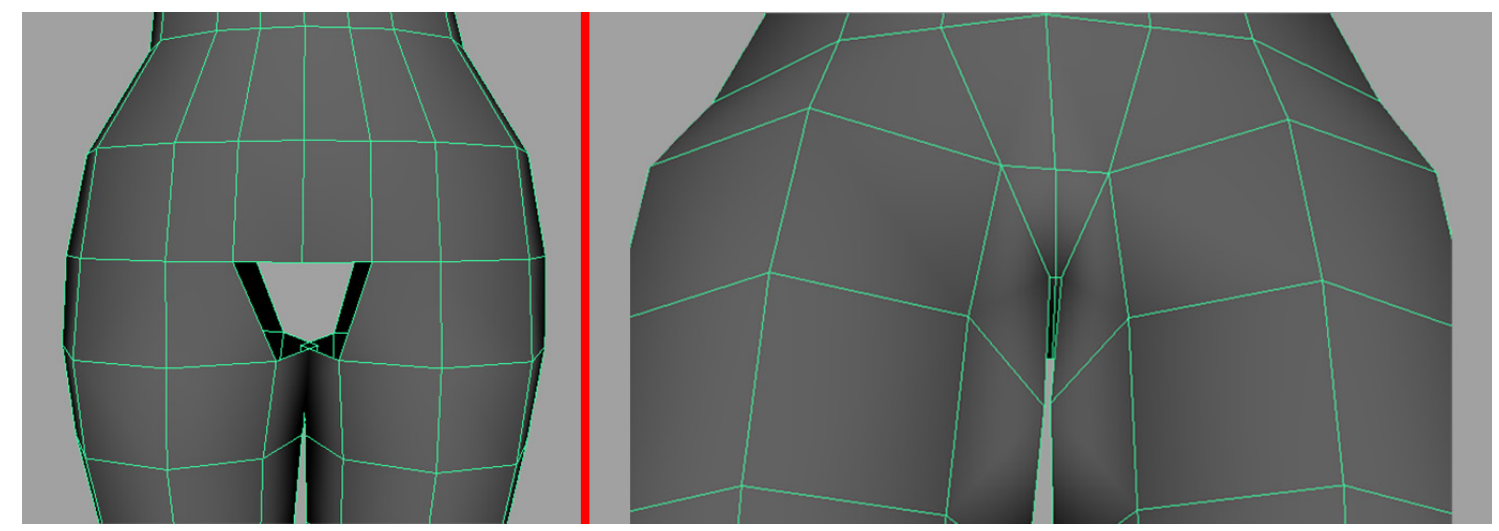

Figure 3.33: Torso-Leg Bridging. Left: partial bridging of the hips (Note the overlap between legs). Right: overlapping solved and middle surface constructed

After combining the torso and legs, the system implements a series of quadratic Bezier curves (equation 10) to smooth the geometry of the hips. As seen in Figure 3.34, similar to Figure 3.29, control points and smoothed vertices are color-coded. However, $t$ is calculated as follows:

$$
t=d_{p} / d
$$

where $d$ is the length of the $3 \mathrm{D}$ line $l$ from $p_{0}$ to $p_{2}$, and $d_{p}$ is the distance between $p_{0}$ and the closest point, in $l$, to the vertex to be smoothed. Figure 3.35 shows the hips after smoothing. 


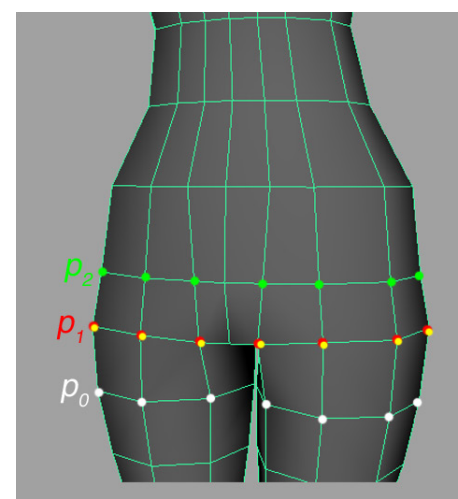

(A)

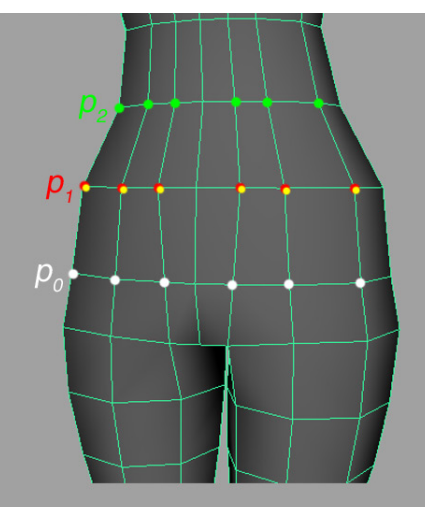

(B)

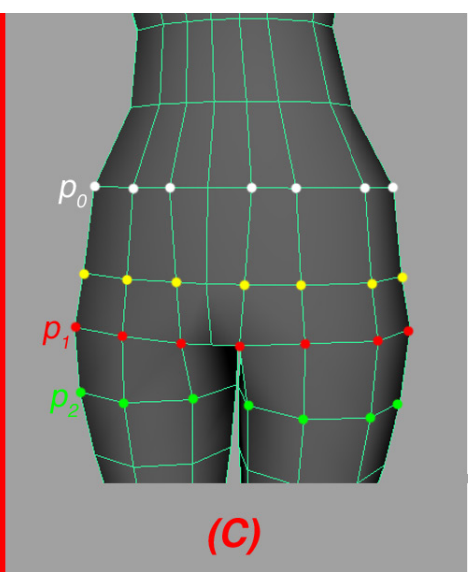

(C)

Figure 3.34: Torso-Leg Smoothing. Smoothing in (B) is only applied to front vertices

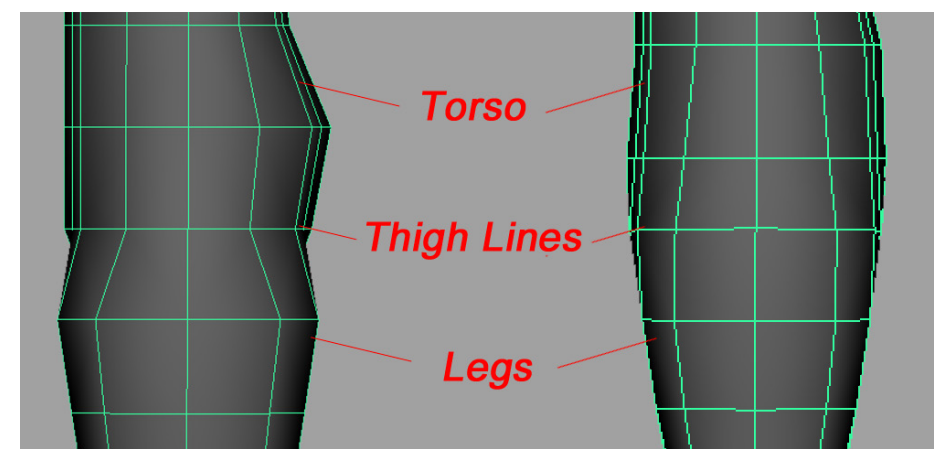

Figure 3.35: The hips before and after smoothing (side view)

In addition, the system curves two edges around the hips to follow the joint and muscle structure. As illustrated in Figure 3.36, repositioning vertices is done by rotation from the origin $\mathrm{C}$. This process affects the $\mathrm{y}$ (height) values of vertex coordinates; $\mathrm{x}$ and $\mathrm{z}$ (width and depth) values remain the same. For maximum rotation angle $\max _{1}$, the system first finds the distance $d$ from $a$ to $b$ then calculates $e$ as follows:

$$
e=a+0.6 d
$$

If $e$ is lower than $F$, the point that results from a 45-degree rotation of the line $C G$, (which in Figure 3.36 is true) then $\max _{1}$ is the angle between $C e$ and $C G$. Otherwise, $\max _{1}$ is 45 degrees. Second, vertex $J$ is centered between $K$ and $C$, and $\min _{1}$ is calculated as the 
angle between $C G$ and $C N$, where $N$ is the end of a horizontal line extended from the newly centered $J$.

As shown, vertices of the upper curve are repositioned using $\max _{1}$ and $\min _{1}$. For the lower curve, $\min _{2}$ is set to zero degrees, and $\max _{2}$ is calculated as follows:

$$
\max _{2}=0.9\left(\min _{1}+0.2 r_{1}\right)
$$

where

$$
r_{1}=\max _{1}-\min _{1}
$$

Table 3.3 lists front vertices and their associated angles of rotation.

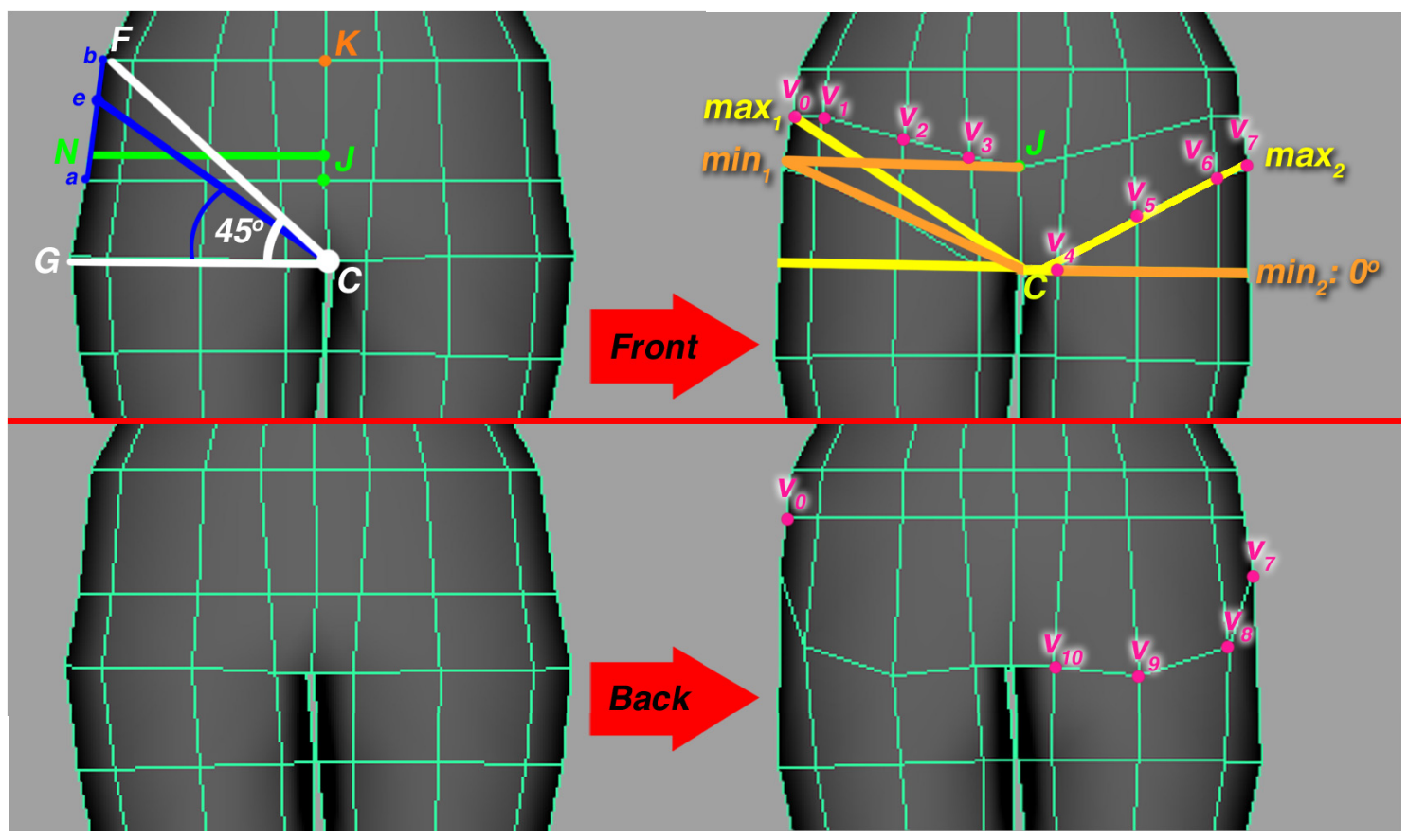

Figure 3.36: Lower Torso Curves 
Table 3.3: Upper \& Lower Curve Rotation Angles (Front)

\begin{tabular}{|c|c|}
\hline Vertex & Rotation Angle (from CG) \\
\hline $\mathbf{V}_{0}$ & $\max _{1}$ \\
\hline $\mathbf{V}_{1}$ & $\max _{1}$ \\
\hline $\mathbf{V}_{2}$ & $\min _{1}+0.55 r_{1}$ \\
\hline $\mathbf{V}_{3}$ & $\min _{1}+0.2 r_{1}$ \\
\hline $\mathbf{V}_{4}$ & $\min _{2}$ \\
\hline $\mathbf{V}_{5}$ & $\min _{2}+0.5 r_{2}$ \\
\hline$V_{6}$ & $\min _{2}+0.9 r_{2}$ \\
\hline \multirow[t]{3}{*}{$\mathbf{V}_{7}$} & $\max _{2}$ \\
\hline & $r_{1}=\max _{1}-\min _{1}$ \\
\hline & $r_{2}=\max _{2}-\min _{2}$ \\
\hline
\end{tabular}

As seen in Figure 3.36, the back vertices are structured differently. Vertices of the upper curve are aligned horizontally or, in other words, they are all rotated $\max _{1}$ degrees from $C G$. On the other hand, Table 3.4 lists the back vertices, of the lower curve, and their rotation angles using $\min _{2}$ and $\max _{2}$. Figure 3.37 shows the final restructuring result.

Table 3.4: Lower Curve Rotation Angles (Back)

\begin{tabular}{c|c}
\hline Vertex & Rotation Angle (from CG) \\
\hline $\mathbf{V}_{\mathbf{8}}$ & $\min _{2}+0.2 r_{2}$ \\
$\mathbf{V}_{\mathbf{9}}$ & $\min _{2}-0.1 r_{2}$ \\
\hline
\end{tabular}




\begin{tabular}{c|c}
\hline $\mathbf{V}_{\mathbf{1 0}}$ & $\min _{2}$ \\
& $r_{2}=\max _{2}-\min _{2}$ \\
\hline
\end{tabular}

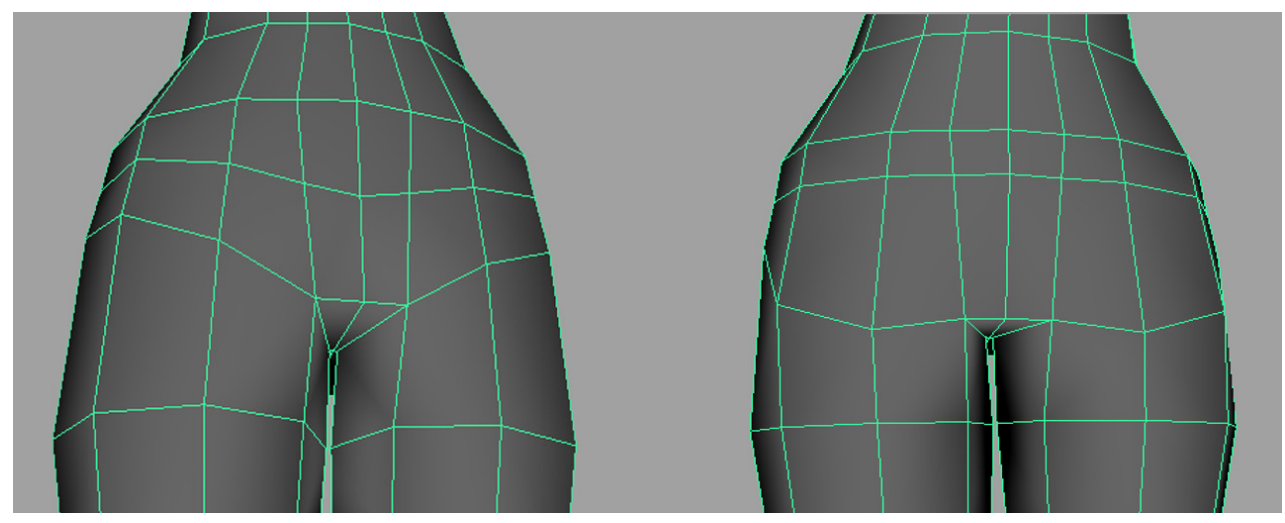

Figure 3.37: Lower Torso Restructuring in front (left) and back (right)

\subsubsection{Combining Shoulders \& Torso}

Connecting shoulders to the torso is different from the process of connecting the hip and the neck. Because the top shoulder line is already aligned with the upper torso lines from both sides (Figure 3.25) the system does not bridge between them with a surface. Instead, it averagely merges aligned shoulder and upper torso vertices, after deleting torso side faces that are bounded by the vertices to merge (Figure 3.38). 


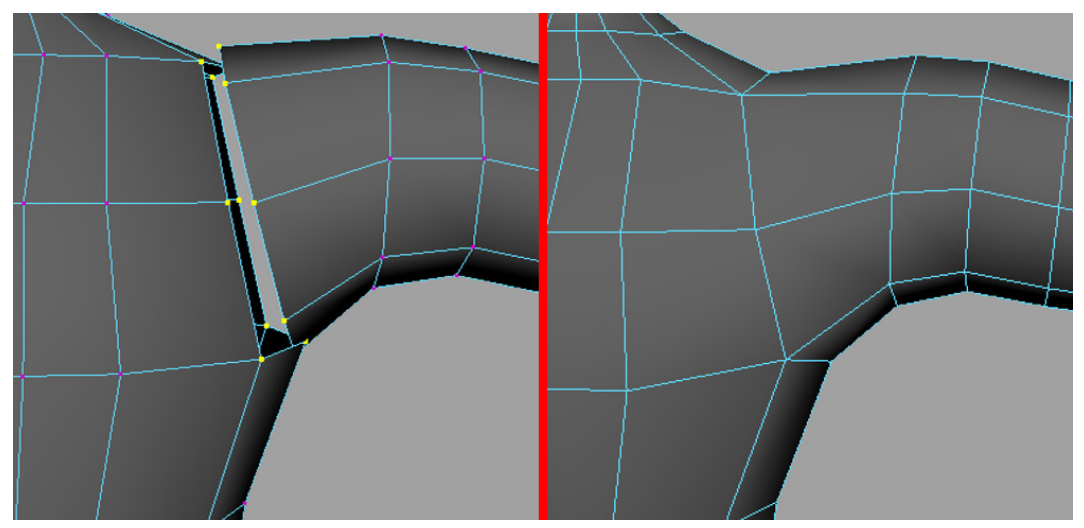

Figure 3.38: Combining Shoulders \& Torso. Left image demonstrates the aligned yellow vertices of the shoulder and torso, which the system averagely merges (right), after deleting bounded faces

After merging, the system centers the arms, in the z-axis, in accordance with the upper torso so that shoulders are not curved forwards or backwards. In addition, as seen in Figure 3.39, two shoulder back vertices are averaged for a smoother result.

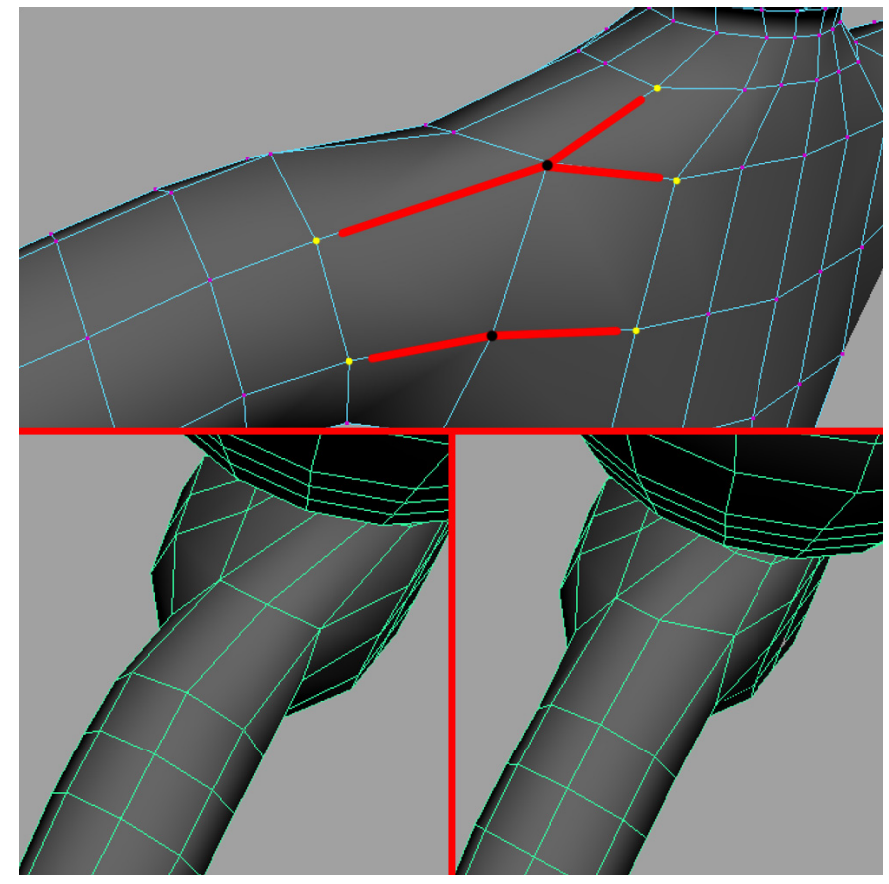

Figure 3.39: Shoulder Adjustment. Top image illustrates the two back vertices (black) adjusted as averages of adjacent ones (yellow). Bottom left image is before centering arms and averaging vertices. Bottom right illustrates the final result 
Combining the shoulders and upper torso is the last process the system automatically applies to the character mesh, producing a complete mesh that consists of head, torso, arms and legs, all connected together, and smoothed for a better appearance and enhanced geometry. Our algorithm strives to follow the standard techniques and guidelines in modeling human characters, producing suitable geometry and topology details that conform to the recommended methods. Figure 3.40 shows a complete 3D mesh of the character in front, side and perspective views.

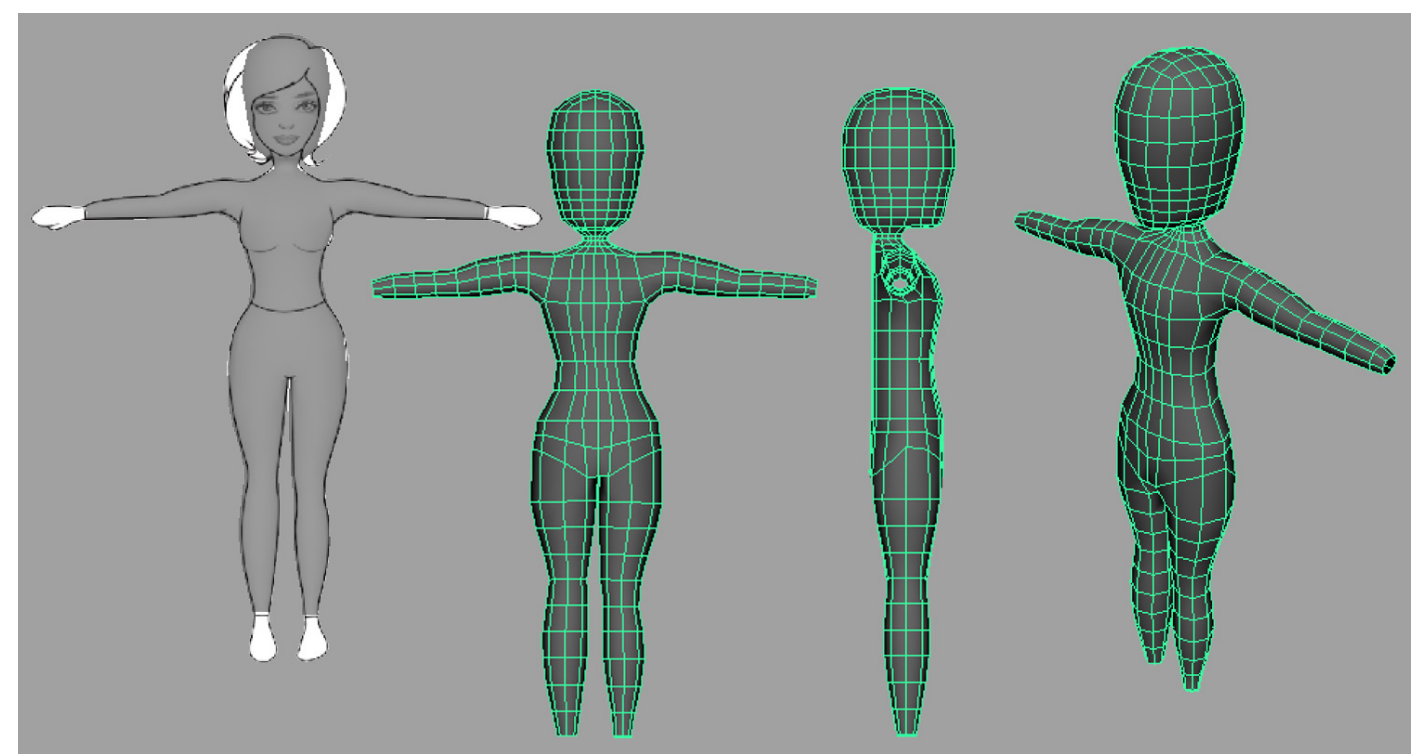

Figure 3.40: The complete 3D character mesh. From left to right, the first two are in front view, aligned with the character sketch (left). The third and fourth are in side and perspective views, respectively.

In this Subsection, we showed step-by-step results to demonstrate how our algorithm works. However, each step of the 3D construction process, as well as the transition from each step to the next is automatically done by the system, which only displays the final result, seen in Figure 3.40, to the user. 
In the next chapter, we introduce the method and results of a user study we conducted to test the performance of our system, and get feedback from 3D modelers regarding their experience with the UI, and evaluation of the mesh. In addition, Appendix B shows some results of the system using different character sketches. 


\section{Experiments and Results}

We conducted a user study to test our system's ability to process a variety of sketched characters. Moreover, the study was done to get feedback and mesh evaluation from 3D modelers, and to evaluate the speed of our algorithm. Users were given instructions on how to use the UI to input the required points on the character. Then, once a 3D model is constructed, they were asked to fill a questionnaire about their user experience with the system as well as their evaluation of the automatically constructed model. Meanwhile, 2D and 3D processing time durations were recorded.

\subsection{Objectives of the Study}

An important goal of our user study was to test the system's rate of success in completing both $2 \mathrm{D}$ and $3 \mathrm{D}$ processes of different character sketches, and to measure the average time it takes to complete those tasks. We were also eager to know if 3D modelers would be interested in automating the basic geometry construction of the character, given a few conditions that we explain in our results and discussion.

Finally, we conducted the user study to get feedback from 3D modelers regarding their user experience with the system's UI, as well as their evaluation of the geometry and topology of the produced mesh, and our approach in smooth combining body parts.

\subsection{User Study Method}

Participants were asked to bring a character sketch that conforms to the requirements we listed in Section 3.3. At the start of the user study session, participants 
first read and signed a consent form (Appendix C). We then demonstrated to the participant how the system's UI works, and showed them how to input the required points on the character, using an example sketch for illustration. After that, we proceeded with the study as follows:

- Using their character sketches, participants used the UI to input the 10 required points on the character, and had the system automatically produce the $3 \mathrm{D}$ character mesh.

- Time durations of user interaction, 2D sketch processing and 3D mesh construction were logged by the system.

- Participants were provided with a questionnaire to fill (Appendix D), while we left the 3D mesh at their presence to be able to check it whenever they needed.

\subsection{Hardware}

The system was run on a Mac OS X, with a $2.6 \mathrm{GHz}$ Intel Core i5 processor, and an 8 GB memory. The average time it took the system to complete both sketch processing and mesh construction was $\mathbf{1 1 . 5}$ seconds, with a standard deviation of 1.52 .

\subsection{Participants}

The total number of people who participated in the user study is 15 , all below the age of 40 . The system succeeded in producing a mesh with 14 out of the 15 participant sketches. Therefore, in order to analyze processing times, user experience, and mesh evaluation, we excluded data of the participant whose sketch was not processed by the 
system. However, in the limitations section of the next chapter, we discuss reasons of failed processing including the user study case.

\subsubsection{Levels of Experience}

As shown in Figure 4.1, users rated their level of experience in 3D modeling generally, and in character modeling particularly. In 3D modeling, 7\% (1 out of 14) said they never modeled before, $29 \%$ are beginners, $21 \%$ are intermediate, $36 \%$ are advanced, and $7 \%$ are expert. On the other hand, in character modeling, 14\% (2 out of 14 ) said they never modeled characters before, $43 \%$ are beginners, $29 \%$ are intermediate, and $14 \%$ are advanced.

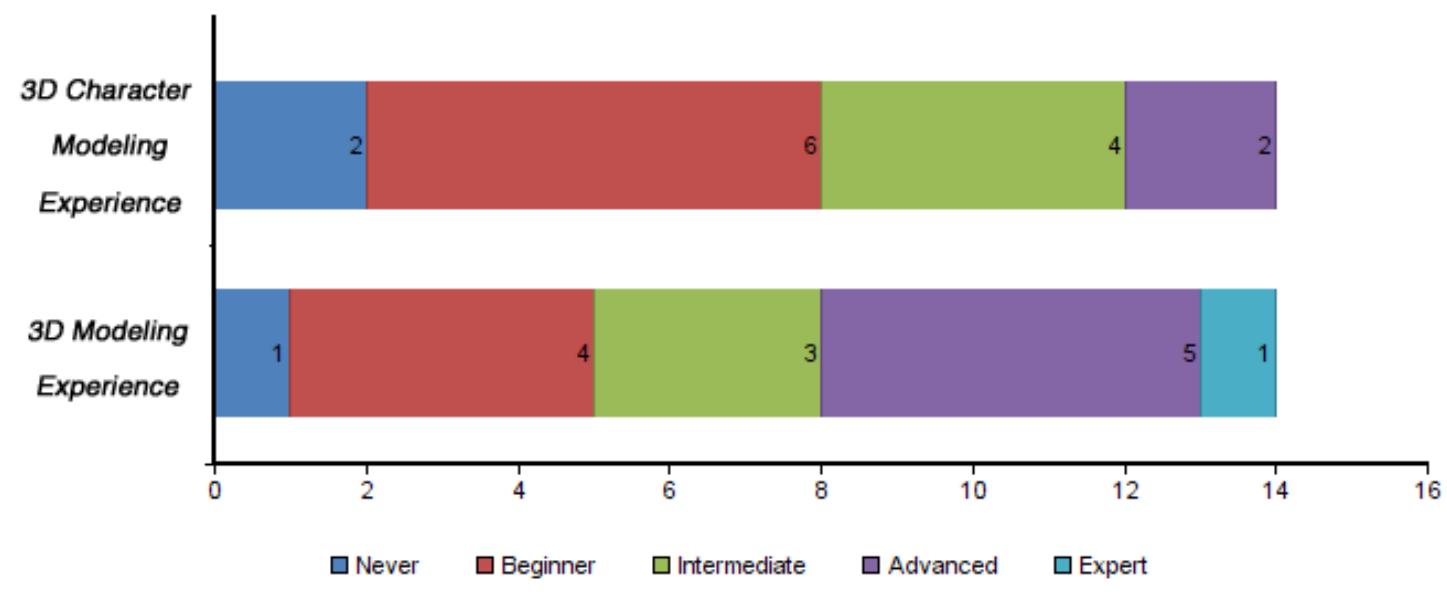

Figure 4.1: Users' 3D Modeling Experience

In addition to modeling experience, users were asked to approximate the time it would take them to build a similar basic geometry to the character mesh produced by the system. As seen in Figure 4.2, all advanced character modelers said it takes them 30 minutes to an hour to create a similar mesh, while answers varied amongst intermediate and beginner modelers. 


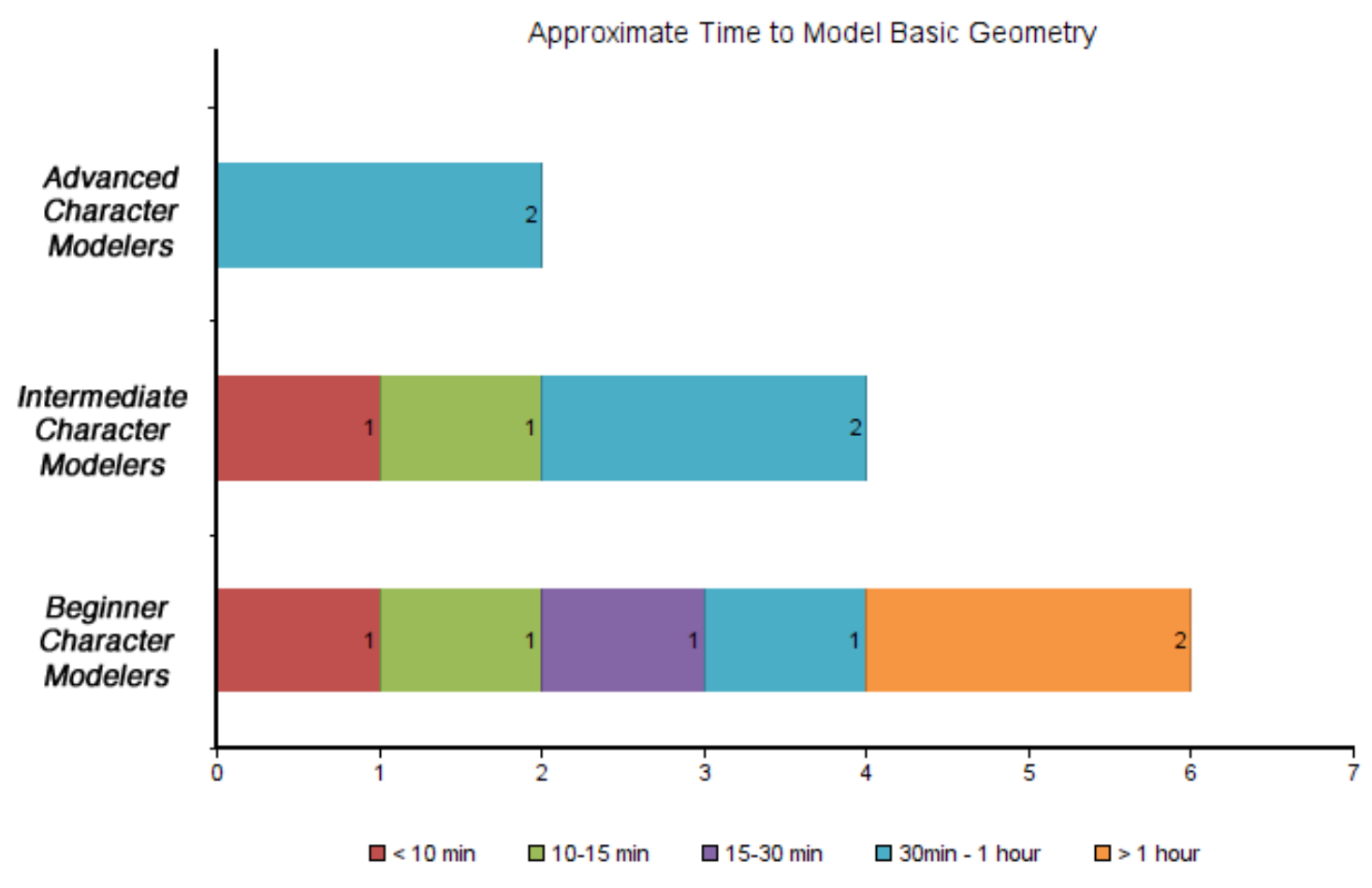

Figure 4.2: Users' estimated time to manually create the same basic geometry created by the system

\subsubsection{Automatic Vs. Manual Modeling}

Users were asked about their preference when it comes to blocking out the character mesh. The first question they were asked was general; "How would you like to go from a sketch to a $3 \mathrm{D}$ character?" and they got to choose between "to get the basic geometry automatically generated", "manually" or "it depends" (Figure 4.3). Users who said "it depends" expressed their concern that the automatic basic geometry might not have the same "structure" or "details" they want, although more than $50 \%$ agreed that automatically generating the geometry would save them time. 


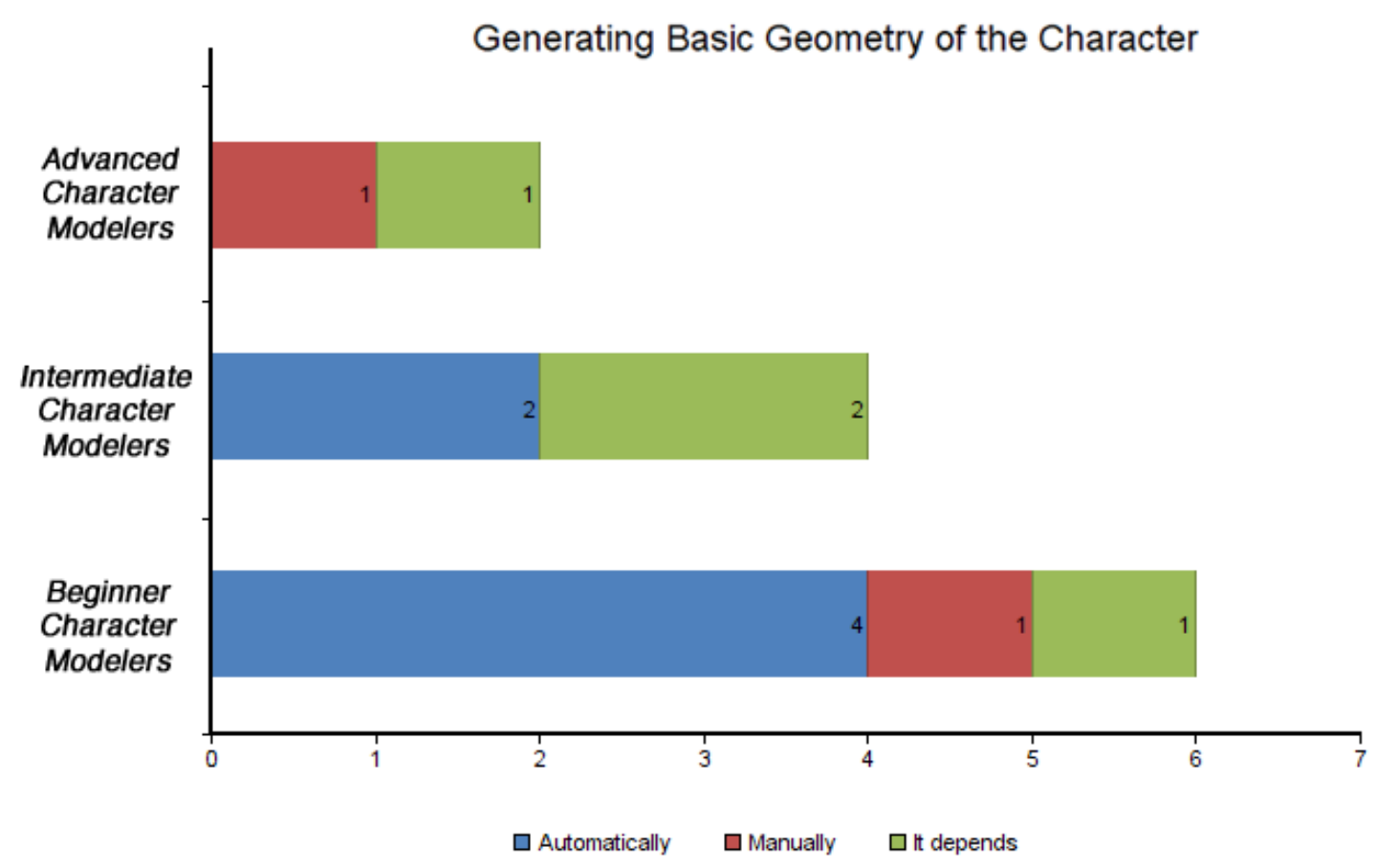

Figure 4.3: Users' preferred method to create the 3D basic geometry of the character

In addition, users were asked about their opinion on automatic modeling if the system takes the same amount of time it takes them to manually block out the character mesh (Figure 4.4). This question was to evaluate the importance of speeding up the character modeling process, to 3D modelers. Users who said it depends focused on the "quality" of the automatic mesh, which has to match that of a manually modeled mesh. On the other hand, some expressed their interest in automatic modeling only if the character is "simple" or "not important". 


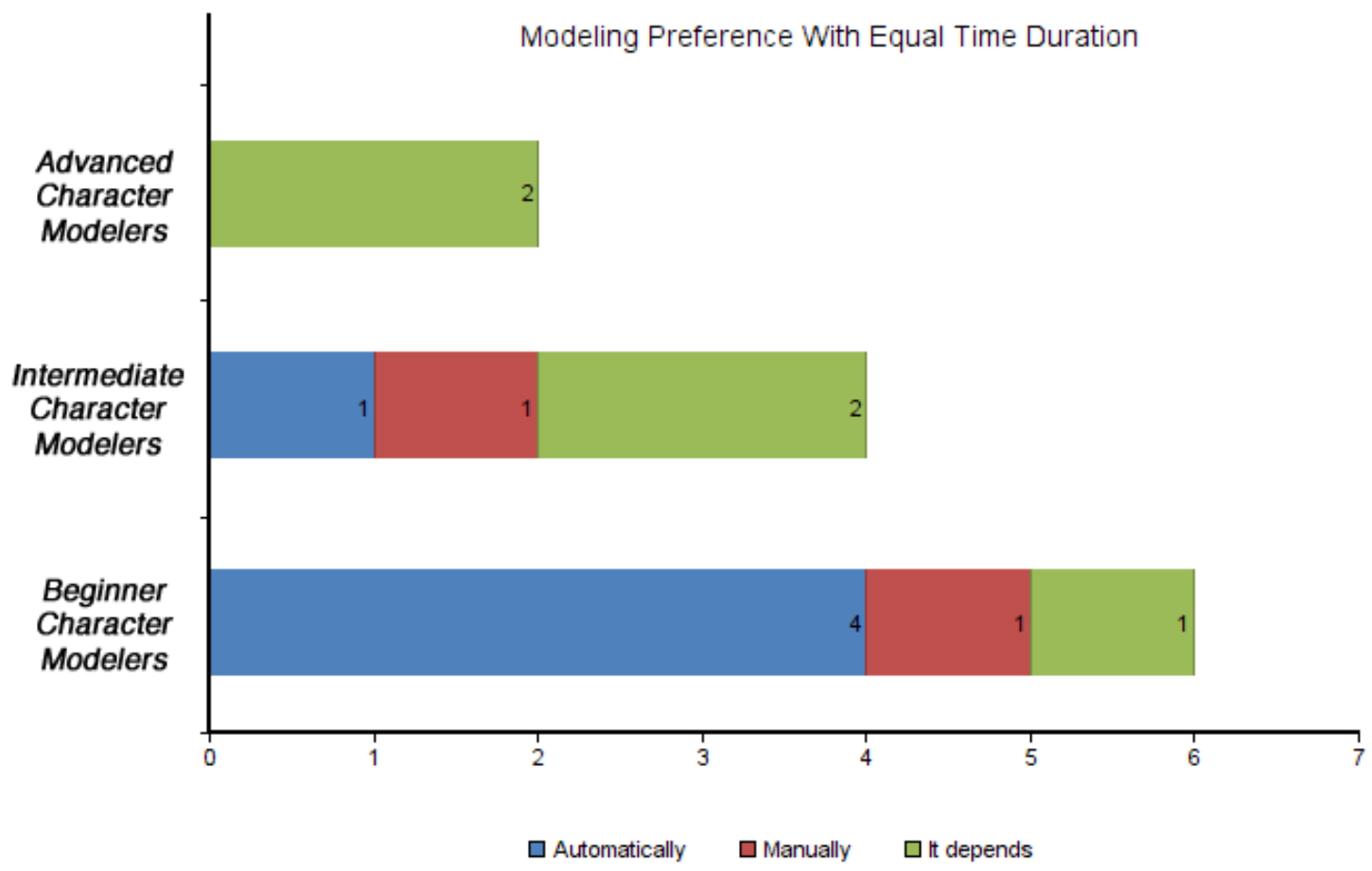

Figure 4.4: Users' preference between automatic and manual modeling if they take the same amount of time

\subsection{Results}

\subsubsection{System Evaluation}

Users were asked about their opinion regarding the amount of specifications, required by the system, in a character sketch (listed in Section 3.3). The rating scale was "too little", "not enough", "enough", "a lot" and "too much". As shown in Figure 4.5, results include users who never modeled characters before. $29 \%$ said the specifications are not enough, suggesting that an added side-view sketch would create better depth results. $57 \%$ said the amount was enough, expressing that the requirements are "very reasonable", "perfectly acceptable" and "straightforward". 14\% said a lot because they limit the flexibility to accept additional details like "weapons or other pieces of armor". 
Users were also asked to rate the amount of input required by the system, namely the 10 points input on the character sketch by the user. As shown in Figure 4.5, 7\% (1 out of 14) said input was too little, $86 \%$ said enough, and $7 \%$ said too much because input points can, instead, be reduced and mirrored when using symmetric characters.

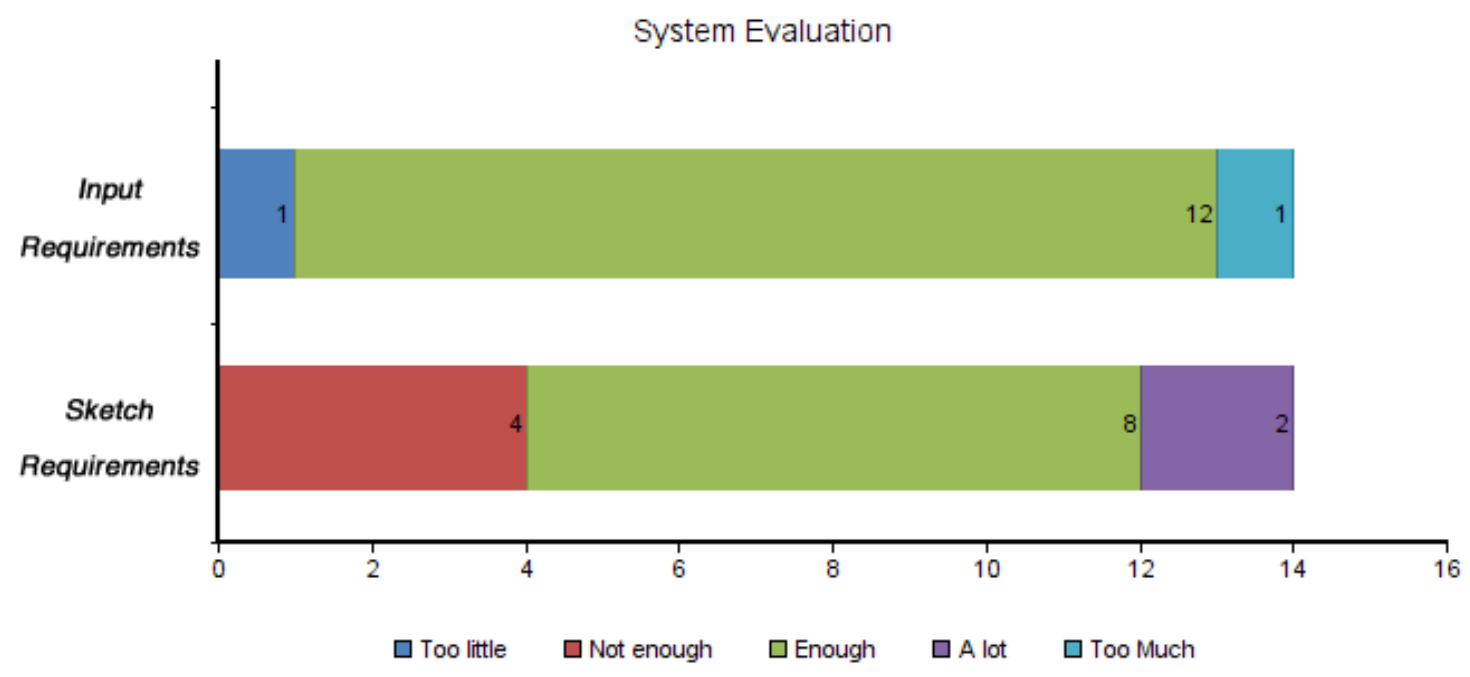

Figure 4.5: Users' evaluation of sketch and input requirements

Users were then asked to rate the ease of learning/understanding the UI, and interacting with it. The two statements are "understanding/learning how to use the system was easy", and "interacting with the system was easy". In addition, they were asked if they thought the system generated the mesh quickly. The rating scale to those 3 questions was: "strongly disagree", “disagree", "neutral", "agree", and "strongly agree". As shown in Figure 4.6, results include users who never modeled characters before. $64 \%$ strongly agreed that understanding and learning the system was easy, and 36\% agreed. Meanwhile, 50\% strongly agreed that interacting with the system was easy, and 50\% 
agreed. On the other hand, $64 \%$ strongly agreed that the system generated the mesh quickly, and $36 \%$ agreed.

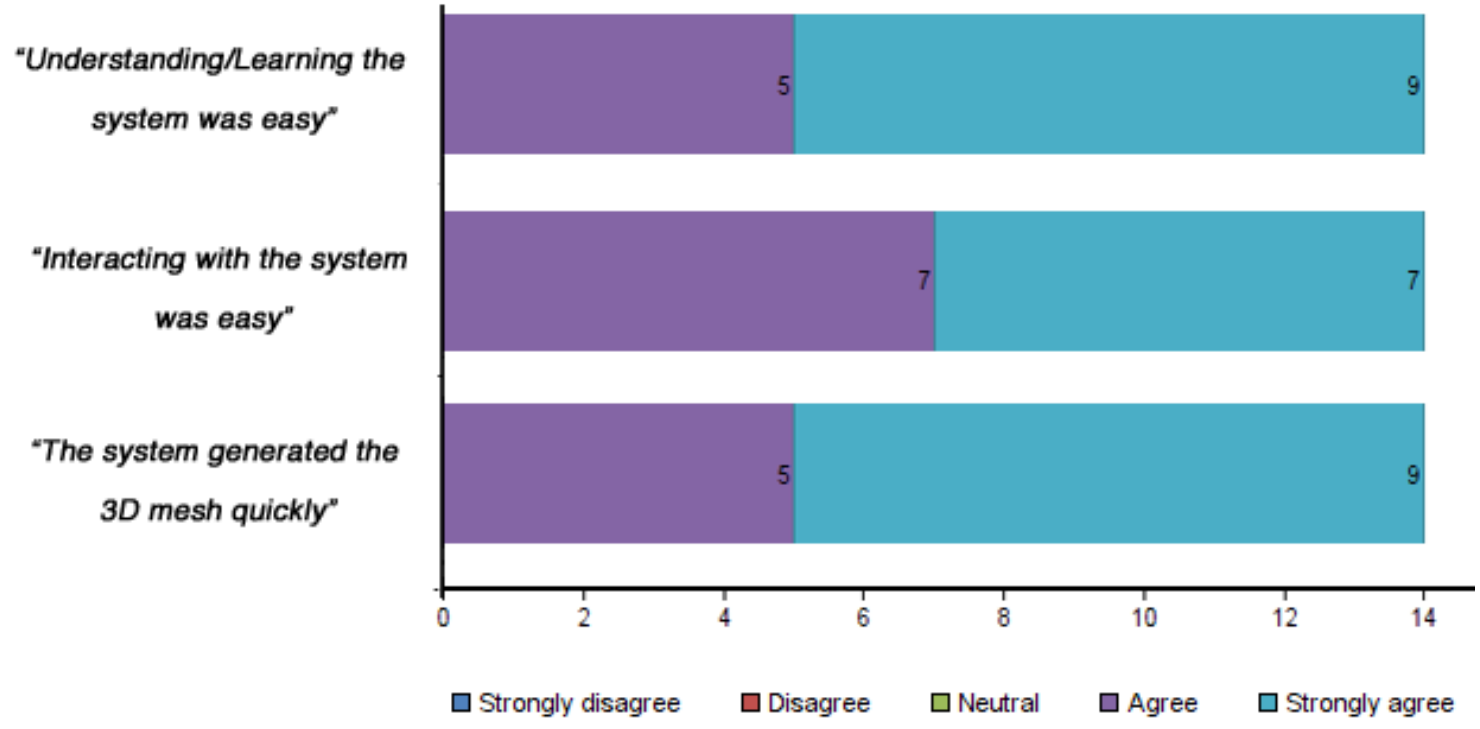

Figure 4.6: UI learning/interactivity \& speed evaluation

\subsubsection{Mesh Evaluation}

In this part of the questionnaire, users were asked to evaluate the generated mesh. The first question was a general rate of the mesh (Figure 4.7), the scale of which was "Very poor", "Poor", "Fair", "Good", and "Very good". The second question was to rate the depth of the mesh with the following statement: "The approximated depth of the mesh is close to how I think the depth of this character should be" (Figure 4.8). The rating scale was from "strongly disagree" to "strongly agree". 


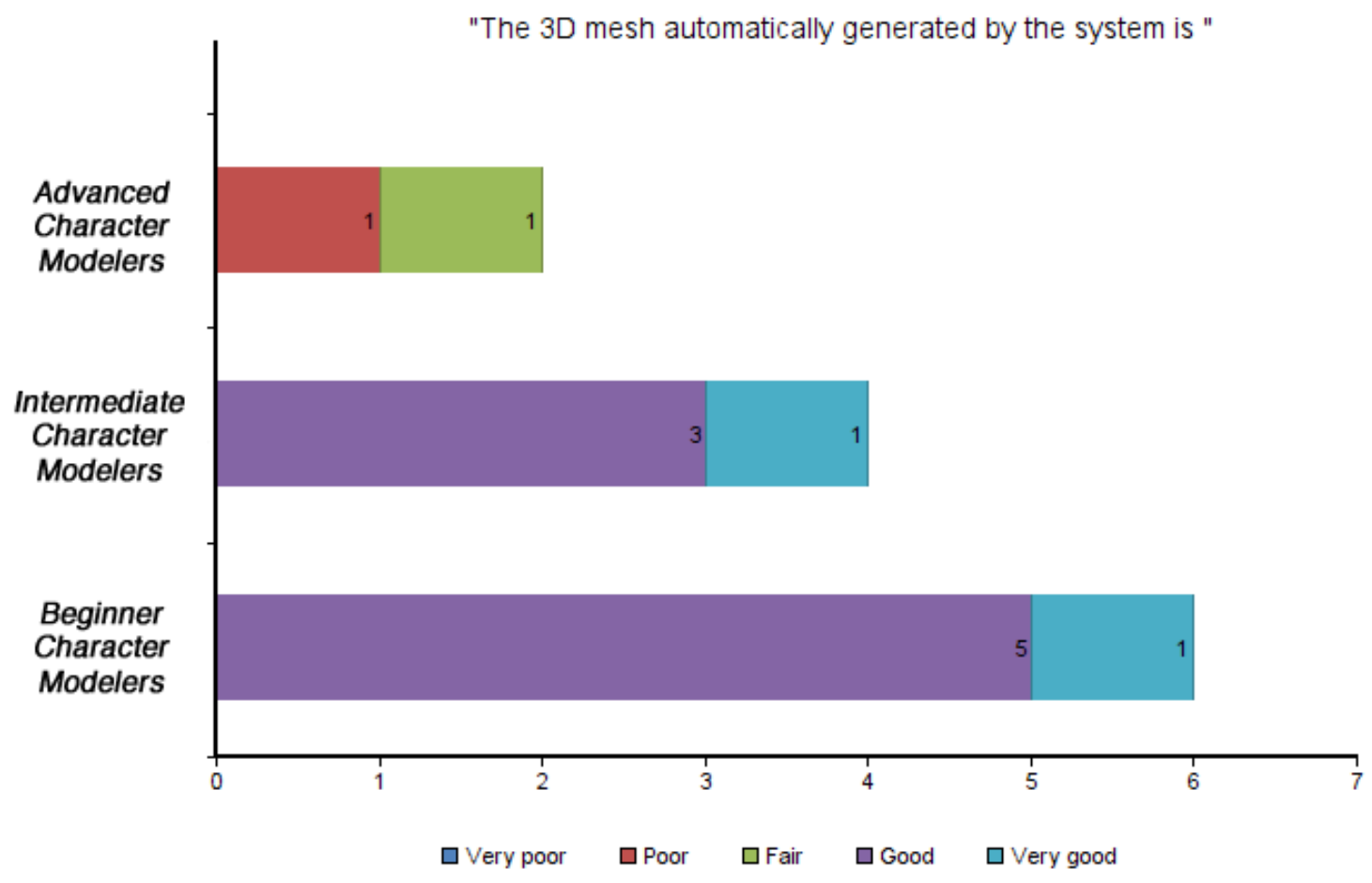

Figure 4.7: General Mesh Evaluation

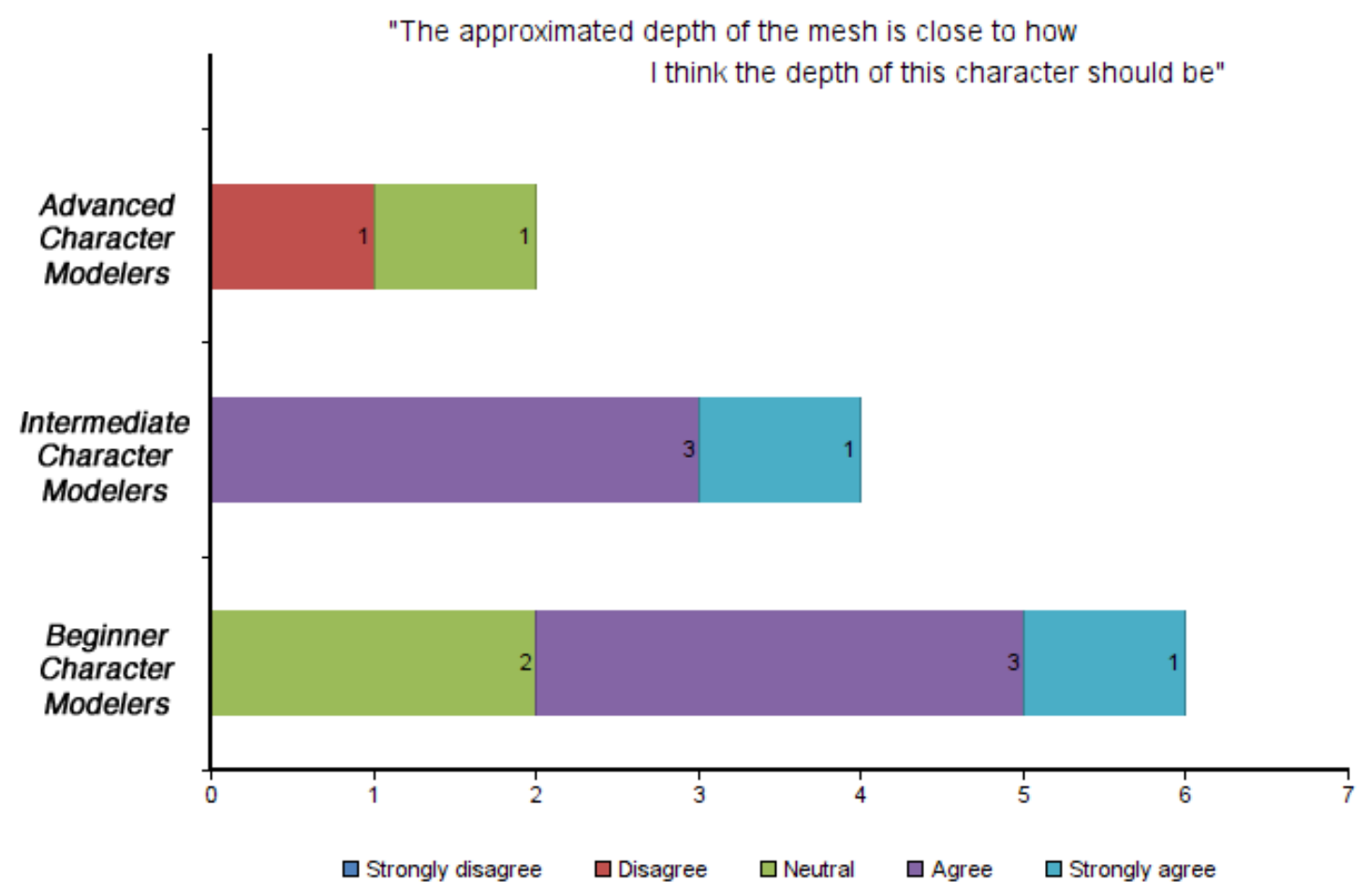

Figure 4.8: Mesh Depth Evaluation 
Users were then asked to evaluate 5 aspects of the 3D mesh. The first one is the geometry of the mesh, which refers to "the consistency in polygon type (i.e. quads or 4sided), and the absence of mesh artifacts". The second aspect is the topology of the mesh, which refers to "how smoothly edge loops wrap around the mesh and how evenly spaced those edge loops are". The 3 other aspects are average geometry-topology evaluations of joined body parts, namely the neck, shoulders and pelvis. The rating scale was from 1 (very poor) to 5 (very good). Table 4.1 shows the average (mean) and median of user ratings.

Table 4.1: Average \& median of mesh geometry \& topology evaluation (1 to 5 scale)

\begin{tabular}{c|c|c|c|c|c|c|}
\cline { 2 - 7 } & \multicolumn{2}{c|}{$\begin{array}{c}\text { Beginner } \\
\text { Character } \\
\text { Modelers }\end{array}$} & \multicolumn{2}{c|}{$\begin{array}{r}\text { Intermediate } \\
\text { Character } \\
\text { Modelers }\end{array}$} & \multicolumn{2}{c|}{$\begin{array}{r}\text { Advanced } \\
\text { Character } \\
\text { Modelers }\end{array}$} \\
\cline { 2 - 7 } & Mean & Median & Mean & Median & Mean & Median \\
\hline Geometry of the Mesh & 4.3 & 4 & 5 & 5 & 4.5 & 4.5 \\
\hline Topology \& Edge Flow of the & 4.2 & 4 & 4.5 & 5 & 3 & 3 \\
\hline Mesh & & & & & & \\
\hline Geometry \& Topology of Neck & 4 & 4 & 3.25 & 4 & 3 & 3 \\
\hline Seometry \& Topology of & 4.17 & 4 & 3.75 & 3.5 & 2.5 & 2.5 \\
\hline
\end{tabular}

Finally, users were asked if they would use the mesh to build their 3D character. Including users who never modeled before, $86 \%$ (12 out of 14 ) said yes, and $14 \%$ said no (Figure 4.9). A user who said no expressed that they would use some parts of the mesh. Another expressed that their current modeling workflow is more advanced than starting with the basic geometry produced by the system, and that using it would be a "step back". 
"I would use this mesh to build my 3D character"

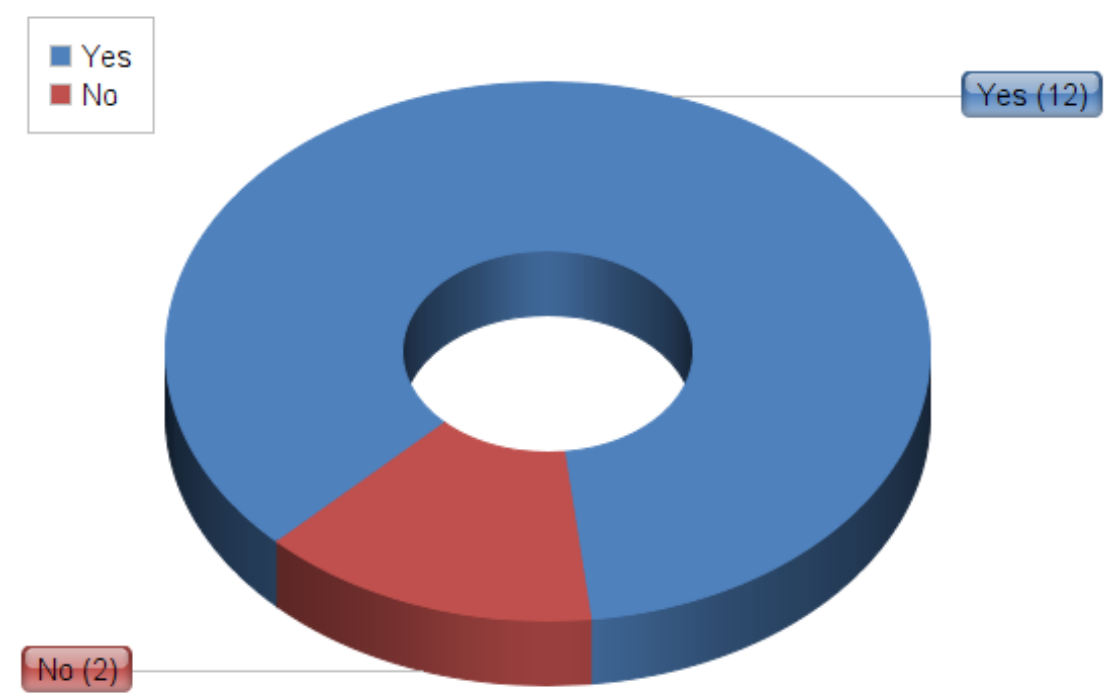

Figure 4.9: Users' willingness to use the automatically generated mesh

Overall, comments about the system results were positive, and some suggestions were given by users to improve the flexibility and accuracy of the system. Users' description of the system included "very useful", "quick", "helpful and efficient", and “accurate size estimation". They suggested "more depth control”, "better topology", and making the system "more customizable".

\subsubsection{Correlations}

A Pearson Correlation test was conducted to assess the relation between users' character modeling experience and mesh evaluation results. Table 4.2 shows the highlighted significant correlations, which are negative. The results suggest that the more experienced the $3 \mathrm{D}$ modeler is, the poorer their rating of the mesh is likely to be, and the less likely they are going to use it. In addition, the results suggest that more experienced 
3D modelers are likely to poorly rate the shoulder geometry and topology, an issue that we discuss in our limitations section of the next chapter.

Table 4.2: Pearson Correlation of character modeling experience with mesh evaluation and willingness to use the mesh

\begin{tabular}{|c|c|c|c|c|c|c|c|c|}
\hline & $\mathrm{a}_{1}$ & $a_{2}$ & $a_{3}$ & $\mathrm{a}_{4}$ & $a_{5}$ & $\mathrm{a}_{6}$ & $a_{7}$ & $\mathrm{a}_{8}$ \\
\hline $\begin{array}{l}\text { Character } \\
\text { Modeling } \\
\text { Experience }\end{array}$ & $-.627 *$ & -.380 & .251 & -.366 & -.453 & $-.680 * *$ & -.354 & $-.710 * *$ \\
\hline
\end{tabular}

\subsection{Discussion}

The results of the user study show promising feedback regarding our system's performance and results. All users agreed that the UI is easy to understand and interact with. That stated that it was "straightforward", "visual", "familiar" and "very easy to manipulate", although one user suggested changing the "wizard style interface", and another suggested that it would be easier to "pre-generate" the points on the sketch, and then have the user adjust their locations. Users also agreed that the system generated the mesh quickly. In addition, the majority of users highly rated the geometry and topology of the mesh, and expressed their willingness to use the mesh to construct the character model.

On the other hand, a few users criticized the limitations of the system that produces less accurate meshes of characters wearing non-fitted clothes, and does not recognize additional objects, such as hats and bags. In addition, since the system closely matches the shape and proportions of the character, users suggested that the system be more 
customizable, in order to produce acceptable results even with poorly sketched characters. They also suggested that we improve torso depth results.

As mentioned earlier, our system generated the mesh in an average of $\mathbf{1 1 . 5}$ seconds, including $2 \mathrm{D}$ and $3 \mathrm{D}$ processing. In addition, the average time of user interaction with the system was 2 minutes and 13 seconds, with a minimum of 1 minute and 10 seconds, a maximum of 5 minutes and 25 seconds, and a standard deviation of 69.75 . This makes the approximate average time of using the system to create the mesh $\mathbf{2}$ minutes and $\mathbf{2 5}$ seconds (interaction plus processing time durations). On the other hand, as demonstrated in Section 4.4, users approximated the time of manually constructing a similar mesh varied greatly. We did not find a significant correlation between manual construction time and user experience, and it was interesting to see from the results that the approximated manual time to block out the basic geometry of the character does not necessarily decrease as modelers become more experienced. However, that being said, it is somewhat difficult to measure the actual time it takes modelers to manually construct the mesh. Users approximated how much time it may take them to construct the model, based on their self-evaluated experience with character modeling.

Another method to measure manual construction time would have been to have users manually create the mesh during the study and calculate the time it takes them to do it. However, this method is not necessarily more accurate than self-reporting the approximate modeling duration. Conducting an experiment where users are asked to manually construct the basic geometry can sacrifice the timing accuracy of that process because a timed study session is not similar to the modeler's usual work setting. 
Moreover, it is debatable whether users or even experimenters are able to decide when the manually constructed model is identical to the automatically generated mesh, in terms of level of detail and structure. Thus, it can be difficult to calculate the exact time it takes the modeler to reach the target mesh, without missing some details or adding ones that are not in the automated mesh.

Finally, part of our research questions was to determine if users value the speed of an automatic SBM system. As shown in Section 4.4, users' preference in using an automatic system versus manual modeling did not greatly change when the system hypothetically lost its speed advantage. Novice modelers (beginners) leaned towards automatic modeling regardless of the time it takes, which supports Alhashim's [11] statement about how novice modelers find it less tedious to work with existing meshes rather than starting from scratch. On the other hand, intermediate and advanced modelers were more likely to base their decision to use the automatic system on its capabilities and the specific details it produces in a mesh. 


\section{Conclusions}

\subsection{Thesis Findings}

In this thesis, we proposed a system that automatically creates the basic geometry of a 3D human character using a single-view sketch and minimal user input. The system takes user input through a simplified UI, and uses that input to derive the character's structure. As a result, it automatically constructs and smoothes the basic geometry of a 3D character. Results of eight sketches are presented in Appendix B.

The results of our user study positively answer our research questions. Our goal was to create an algorithm that conforms to the current modeling techniques as well as the geometry and topology that professional modelers recommend. User evaluation of the mesh indicates that our algorithm produces good and usable meshes.

In addition, this system is aimed to facilitate and speed up the character modeling process to novice modelers. The user study conducted to evaluate this system resulted in positive feedback from both beginner and intermediate users who found it helpful and quick to start up the process. 


\subsection{Limitations \& Future Work}

\subsubsection{Robustness \& Accuracy}

\subsubsection{Pixel-Based Tracing}

Tracing the sketch outline is pixel-based, which affects the appearance of the mesh if a sketch image is in low-resolution, or if the drawing is over-traced. This results in a somewhat "wrinkly" mesh. To reduce the effects of pixel-based tracing, we used high-resolution images of at least 650 pixels in width and 720 pixels in height. Additionally, we implemented a smoothing method to reduce over-tracing side effects. However, an improvement would be to convert our method to vector-based tracing and integrate a better smoothing technique that eliminates unnecessary details from the outline.

\subsubsection{Topology \& Edge Flow}

We based our mesh-building algorithm on standard modeling techniques to create suitable topology. However, based on feedback from 3D modelers, the edge flow around joined areas can be improved for smoother results. A future work can be conducted to focus on and enhance the body combining and smoothing part of the system.

\subsubsection{Upper Torso Structure}

Our proposed system uses the curve detection algorithm to detect underarms and, based on that, it derives the clavicle and shoulder lines. Some issues occurred when the character's arms were not fully extended in a t-pose, which affected the appearance of shoulders in some meshes, which also explains the negative correlation we got in our 
study results in Chapter 4. A future work would be to improve the method of locating the clavicle line based on how far the arms are extended away from the body. On the other hand, when arms are not far enough, the system sometimes generates overlapping geometry between the shoulders and upper torso. A simple improvement would be to integrate an arm rotation method to automatically generate a t-posed character.

\subsubsection{Input Effect}

We aimed to create a system that quickly blocks out the character mesh. To achieve that, the system relies on the accuracy of user input. However, there is no clear definition as to what accurate input is because input locations vary from character to another, and different inputs on the same character produce different results, all of which may be acceptable. For example, different perceptions of where the neck bone starts and ends leads to different input of the two neck points. As a result, the system can produce the same character with different neck lengths and, consequently, a slightly varying upper torso structure.

Varying inputs did not seem to affect the overall appearance of the mesh, as long as the user inputs each point in its "expected" location (e.g., a wrist point cannot be placed on the ankle). However, this issue caused one of our user study cases to fail. The character sketch used in that case had too unrealistic proportions, and was rated by the participant as a very poorly drawn sketch. Even though we aimed to make our system flexible with all types of drawings, it still appeared to be sensitive to user input in some cases, and the failed study occurred when the system was unable to derive upper torso information based on the given points. To verify that our system was, in fact, sensitive to 
input and not completely rejecting the sketch, we tested it with a slightly varied input and were able to generate a mesh.

The system can be improved to verify and adjust input by analyzing the character's body structure in detail with additional sketch processing methods. This improvement may enhance the flexibility of the system, although it may lengthen the overall processing time.

\subsubsection{Torso Depth}

We estimate the depth of the character's torso by combining anthropometric data with a box modeling approach. The torso has a spherical shape adjusted in width by the character's outline, and in depth by visualized anthropometrics and interpolation between the upper and lower torso. These estimates were favored based on empirical evidence, and about $67 \%$ of character modelers agreed or strongly agreed that the depth of the character is how they expected it to be. In addition, our torso depth adjustment algorithm succeeded in producing characters with different waist sizes. However, there is plenty of room for depth improvement, which can be done using more anthropometric measurements.

In addition, to keep a basic geometry, the system generates a uniformly sized structure across the torso and hips, resulting in a flat spine and posterior. Consequently, some users suggested that enhancing the torso details would be a significant improvement to the appearance of the mesh. 


\subsubsection{Head \& Hair Structure}

We used standard measurements to estimate the head dimensions. This approach is aimed to exclude the character's hair from the mesh. However, as results show in Appendix B, there are cases when the system still includes some hair volume in the mesh, while in other cases it excludes parts of the head from the mesh. To reduce the complexity of adjusting head measurements, our system produces simple geometry of the head that is easy to fix manually, as stated by users. However, for more accurate results, besides processing the outline, the system can use internal strokes of the sketched character to trace the hairline and estimate the hair volume for more accurate head dimensions.

\subsubsection{Garment Processing}

As illustrated in the methodology chapter, our algorithm traces the body structure of the character with no regard to garment details, which is why the system expects a character wearing fitted clothing to ensure better results. We only use the character's outline to produce the mesh, and if the character is wearing loose garment, then garment details are interpreted as part of the character's body and, as a result, affect the mesh geometry. Based on user feedback, this approach is not wrong because some modelers prefer to integrate clothes, regardless of how fitted they are, with the character in a single mesh.

However, our results showed that complex garment details might result in unfavorable geometry that appears wrinkly or bumpy. While the low-poly mesh produced by the system allows for quick adjustment, our approach might not be ideal for complex 
garments. An alternative approach would be to integrate the internal strokes of the sketch to distinguish garment details from the human body, in order to produce a smoother mesh. This approach would also be able to recognize when the character is wearing a dress or a skirt and, as a result, infer hidden leg details.

In addition, integrating internal strokes will help the system to infer a more accurate neck width in the presence of a collared top, and perform better underarm detection with loose sleeves. As mentioned earlier, more sketch processing is required to achieve such goals and that may affect how fast the mesh is generated, but our user study suggests that $3 \mathrm{D}$ modelers may tolerate relatively slower processing from a system that can accomplish such advanced goals.

\subsection{Summary}

In conclusion, despite the limitations of our system, results and feedback indicate that our system produces suitable results for non-advanced modelers to start and speed up the modeling process, using an intuitive and easy to use UI. 3D modeling students showed huge interest in using the system, and modeling experts expressed the usefulness of the system to beginners. This proves that our system contributes to the promising direction towards building more familiar and supported SBM systems that can be easily used by modelers. Moreover, suggested future works can help produce more complex meshes for advanced users. 


\section{References}

[1] I. V. Kerlow, The Art of 3-D Computer Animation and Imaging, 2nd ed. New York, NY: John Wiley \& Sons, 2000.

[2] J. Dargie, "Modeling techniques: movies vs. games," ACM SIGGRAPH Comput. Graph., vol. 41, no. 2, p. 2, May 2007.

[3] J. Patnode, Character Modeling with Maya and ZBrush: Professional polygonal modeling techniques, 1st ed. Amsterdam: Focal, 2008.

[4] T. Flaxman, Maya ${ }^{\circledR} 2008$ Character Modeling and Animation: Principles and Practices. Course Technology, 2008.

[5] "Applying scenarios in user-centred design to develop a sketching interface for human modelling and animation," in Proceedings of Eurographics Workshop on Sketch Based Interfaces and Modeling (SBIM06), 2006, pp. 147-156.

[6] D. A. Bowman, E. Kruijff, J. J. LaViola Jr, and I. Poupyrev, 3D user interfaces: theory and practice. Addison-Wesley, 2004.

[7] M. T. Cook and A. Agah, "A survey of sketch-based 3-D modeling techniques," Interact. Comput., vol. 21, no. 3, pp. 201-211, Jul. 2009.

[8] A. Wood, "Behind the Scenes: A Study of Autodesk Maya," Animation, vol. 9, no. 3, pp. 317-332, Nov. 2014.

[9] C. Maraffi, Maya Character Creation: Modeling and Animation Controls, 1st ed. Indianapolis, IN: New Riders, 2004.

[10] M. Habbecke and L. Kobbelt, "An Intuitive Interface for Interactive High Quality Image-Based Modeling," Comput. Graph. Forum, vol. 28, no. 7, pp. 1765-1772, 
Oct. 2009.

[11] I. Alhashim, "Modeling and Correspondence of Topologically Complex 3D Shapes," Jun. 2015.

[12] I. K. Kazmi, L. You, and J. J. Zhang, “A Survey of Sketch Based Modeling Systems," in 2014 11th International Conference on Computer Graphics, Imaging and Visualization, 2014, pp. 27-36.

[13] T. Igarashi, S. Matsuoka, and H. Tanaka, "Teddy," in ACM SIGGRAPH 2007 courses on - SIGGRAPH '07, 2007, p. 21.

[14] A. Nealen, T. Igarashi, O. Sorkine, and M. Alexa, "FiberMesh," ACM Trans. Graph., vol. 26, no. 3, p. 41, Jul. 2007.

[15] B. R. De Araújo and J. A. P. Jorge, "Blobmaker: Free-form modelling with variational implicit surfaces," vol. 12, pp. 17-26, 2003.

[16] A. Andre and S. Saito, "Single-view sketch based modeling," in Proceedings of the Eighth Eurographics Symposium on Sketch-Based Interfaces and Modeling SBIM'11, 2011, p. 133.

[17] E. Entem, L. Barthe, M.-P. Cani, F. Cordier, and M. van de Panne, "Modeling 3D animals from a side-view sketch," Comput. Graph., vol. 46, pp. 221-230, Feb. 2015.

[18] L. Olsen, F. F. Samavati, and J. A. Jorge, "NaturaSketch: Modeling from Images and Natural Sketches," IEEE Comput. Graph. Appl., vol. 31, no. 6, pp. 24-34, Nov. 2011.

[19] L. M. V. Cruz and L. Velho, "A Sketch on Sketch-Based Interfaces and Modeling," in 2010 23RD SIBGRAPI - Conference on Graphics, Patterns and 
Images Tutorials, 2010, pp. 22-33.

[20] L. Olsen, F. F. Samavati, M. C. Sousa, and J. A. Jorge, "Sketch-based modeling: A survey," Comput. Graph., vol. 33, no. 1, pp. 85-103, Feb. 2009.

[21] G. Orbay and L. B. Kara, "Sketch-based modeling of smooth surfaces using adaptive curve networks," in Proceedings of the Eighth Eurographics Symposium on Sketch-Based Interfaces and Modeling - SBIM '11, 2011, p. 71.

[22] C. Koussa and M. Koehl, "A simplified geometric and topological modeling of 3D buildings: combination of surface-based and solid-based representations," in ASPRS 2009 annual Conference: Reflection of the past, vision of the future, 2009.

[23] O. A. Karpenko and J. F. Hughes, "SmoothSketch," ACM Trans. Graph., vol. 25, no. 3, p. 589, Jul. 2006.

[24] O. Karpenko, J. F. Hughes, and R. Raskar, "Free-form sketching with variational implicit surfaces," Comput. Graph. Forum, vol. 21, no. 3, pp. 585-594, Sep. 2002.

[25] A. Alexe, V. Gaildrat, and L. Barthe, "Interactive modelling from sketches using spherical implicit functions," in Proceedings of the 3rd international conference on Computer graphics, virtual reality, visualisation and interaction in Africa AFRIGRAPH'04, 2004, p. 25.

[26] S. Owada, F. Nielsen, K. Nakazawa, and T. Igarashi, "A sketching interface for modeling the internal structures of 3D shapes," in ACM SIGGRAPH 2007 courses on-SIGGRAPH '07, 2007, p. 38.

[27] R. Yang and B. C. Wünsche, "Life-sketch: a framework for sketch-based modelling and animation of 3D objects," in the Eleventh Australasian Conference on User Interface - Volume 106, 2010, pp. 61-70. 
[28] O. Gonen and E. Akleman, "Sketch based 3D modeling with curvature classification," Comput. Graph., vol. 36, no. 5, pp. 521-525, Aug. 2012.

[29] A. Rivers, F. Durand, and T. Igarashi, "3D modeling with silhouettes," in $A C M$ SIGGRAPH 2010 papers on - SIGGRAPH '10, 2010, p. 1.

[30] R. D. Viana, E. R. Nascimento, and R. A. C. Ferreira, "On the Development of a Fully Automatic Methodology to Create Smooth Mesh for 3D Models from Concept Arts," in Proceedings of the 13th Brazilian Symposium on Games and Digital Entertainment, 2014.

[31] I. K. Kazmi, L. You, X. Yang, X. Jin, and J. J. Zhang, "Efficient sketch-based creation of detailed character models through data-driven mesh deformations," Comput. Animat. Virtual Worlds, vol. 26, no. 3-4, pp. 469-481, May 2015.

[32] C. Yang, D. Sharon, and M. van de Panne, "Sketch-based Modeling of Parameterized Objects," EG Work. Sketch-Based Interfaces Model., pp. 63-72, 2005.

[33] K. Xu, H. Zheng, H. Zhang, D. Cohen-Or, L. Liu, and Y. Xiong, "Photo-inspired model-driven 3D object modeling," in ACM SIGGRAPH 2011 papers on SIGGRAPH '11, 2011, p. 1.

[34] B. Li and H. Johan, "Sketch-based 3D model retrieval by incorporating 2D-3D alignment," Multimed. Tools Appl., vol. 65, no. 3, pp. 363-385, Aug. 2013.

[35] H. Pan, Y. Liu, A. Sheffer, N. Vining, C.-J. Li, and W. Wang, "Flow aligned surfacing of curve networks," ACM Trans. Graph., vol. 34, no. 4, pp. 127:1127:10, Jul. 2015.

[36] Y. Zhuang, M. Zou, N. Carr, and T. Ju, "A general and efficient method for 
finding cycles in 3D curve networks," ACM Trans. Graph., vol. 32, no. 6, pp. 110, Nov. 2013.

[37] E. Iarussi, D. Bommes, and A. Bousseau, "BendFields," ACM Trans. Graph., vol. 34, no. 3, pp. 1-16, May 2015.

[38] B. Xu, W. Chang, A. Sheffer, A. Bousseau, J. McCrae, and K. Singh, “True2Form," ACM Trans. Graph., vol. 33, no. 4, pp. 1-13, Jul. 2014.

[39] E. Schweikardt and M. D. Gross, "Digital clay: deriving digital models from freehand sketches," Autom. Constr., vol. 9, no. 1, pp. 107-115, Jan. 2000.

[40] Y. Zhang, J. Zheng, and N. Magnenat-Thalmann, "Example-guided anthropometric human body modeling," Vis. Comput., vol. 31, no. 12, pp. 16151631, Dec. 2015.

[41] A. Johnston, G. Carneiro, R. Ding, and L. Velho, "3-D Modeling from Concept Sketches of Human Characters with Minimal User Interaction,” in 2015 International Conference on Digital Image Computing: Techniques and Applications (DICTA), 2015, pp. 1-8.

[42] C. Mao, S. F. Qin, and D. Wright, "A sketch-based approach to human body modelling," Comput. Graph., vol. 33, no. 4, pp. 521-541, Aug. 2009.

[43] V. Kraevoy, A. Sheffer, and M. van de Panne, "Modeling from contour drawings," in Proceedings of the 6th Eurographics Symposium on Sketch-Based Interfaces and Modeling - SBIM '09, 2009, p. 37.

[44] C. Benabdelkader and Y. Yaser, "chapter Statistical Estimation of Human Anthropometry from a Single Uncalibrated Image," in In Workshop on Biometric Authentication, pp. 200-220. 
[45] Y. Yang and D. Ramanan, "Articulated Human Detection with Flexible Mixtures of Parts," IEEE Trans. Pattern Anal. Mach. Intell., vol. 35, no. 12, pp. 2878-2890, Dec. 2013.

[46] V. Ramakrishna, T. Kanade, and Y. Sheikh, "Reconstructing 3D Human Pose from 2D Image Landmarks,” 2012, pp. 573-586.

[47] S. Zhu, P. Y. Mok, and Y. L. Kwok, "An efficient human model customization method based on orthogonal-view monocular photos," Comput. Des., vol. 45, no. 11, pp. 1314-1332, Nov. 2013.

[48] X. Zhou, J. Chen, G. Chen, Z. Zhao, and Y. Zhao, "Anthropometric body modeling based on orthogonal-view images," Int. J. Ind. Ergon., vol. 53, pp. 2736, May 2016.

[49] X. Han, K.-Y. K. Wong, and Y. Yu, "3D Human Model Reconstruction from Sparse Uncalibrated Views," IEEE Comput. Graph. Appl., 2015.

[50] M. H. Nguyen, B. Wünsche, P. Delmas, and C. Lutteroth, "Modelling of 3D Objects Using Unconstrained and Uncalibrated Images Taken with a Handheld Camera," 2013, pp. 86-101.

[51] P. Buchanan, R. Mukundan, and M. Doggett, "Automatic single-view character model reconstruction," in Proceedings of the International Symposium on SketchBased Interfaces and Modeling - SBIM '13, 2013, p. 5.

[52] P. Selinger, "Potrace: A polygon-based tracing algorithm," 2003.

[53] P. Ratner, 3-D human modeling and animation, 3rd ed. New York, NY, USA: John Wiley \& Sons, 2012.

[54] M. Bessmeltsev, W. Chang, N. Vining, A. Sheffer, and K. Singh, "Modeling 
Character Canvases from Cartoon Drawings," ACM Trans. Graph., vol. 34, no. 5, pp. 1-16, Nov. 2015.

[55] M. E. Celebi, "Improving the performance of k-means for color quantization," Image Vis. Comput., vol. 29, no. 4, pp. 260-271, Mar. 2011.

[56] N. Chansri and P. Koomsap, "Automatic single-line drawing creation from a paper-based overtraced freehand sketch," Int. J. Adv. Manuf. Technol., vol. 59, no. 1-4, pp. 221-242, Mar. 2012.

[57] Y.-L. Lin and M.-J. J. Wang, "Automated body feature extraction from 2D images," Expert Syst. Appl., vol. 38, no. 3, pp. 2585-2591, Mar. 2011.

[58] "Head Proportions." [Online]. Available: http://vilppustore.com/vilppublog/2011/05/head-drawing-proportions/. [Accessed: 05-Mar-2016].

[59] A. Lopez, E. Garces, and D. Gutierrez, "Depth from a single image through user interaction," in $C E I G, 2014$, pp. 1-10.

[60] O. Villar, Learning Blender: A Hands-On Guide to Creating 3D Animated Characters. Boston: Addison-Wesley, 2014.

[61] Anthropometric Source Book Volume II: A Handbook of Anthropometric Data. NASA Reference Publication 1024, 1978. 


\section{Appendix A: Permission to Use Copyrighted Material Form}

Title of thesis: A System that Automatically Creates Basic Geometry of a 3D Human

Character using a Single Front-View Sketch and Minimal User Input

Degree: Master's in Computer Science

Graduating year: 2016

Permission is hereby granted to: Shaikah Bakerman

To reproduce the following in the thesis:

\section{- A character drawing}

I am also aware that the author of this thesis will also be granting nonexclusive licenses to Carleton University Library and Library and Archives

Canada. Licenses are available at:

Carleton University Library:

http://www2.carleton.ca/fgpa/ccms/wp-content/ccms-files/Licence-to-

Carleton.pdf

Library and Archives Canada:

http://www.collectionscanada.gc.ca/obj/s4/f2/frm-n159-2.pdf

Signature of copyright holder:

Name of copyright holder:

Date:

Contact information: 


\section{Appendix B: System Results}

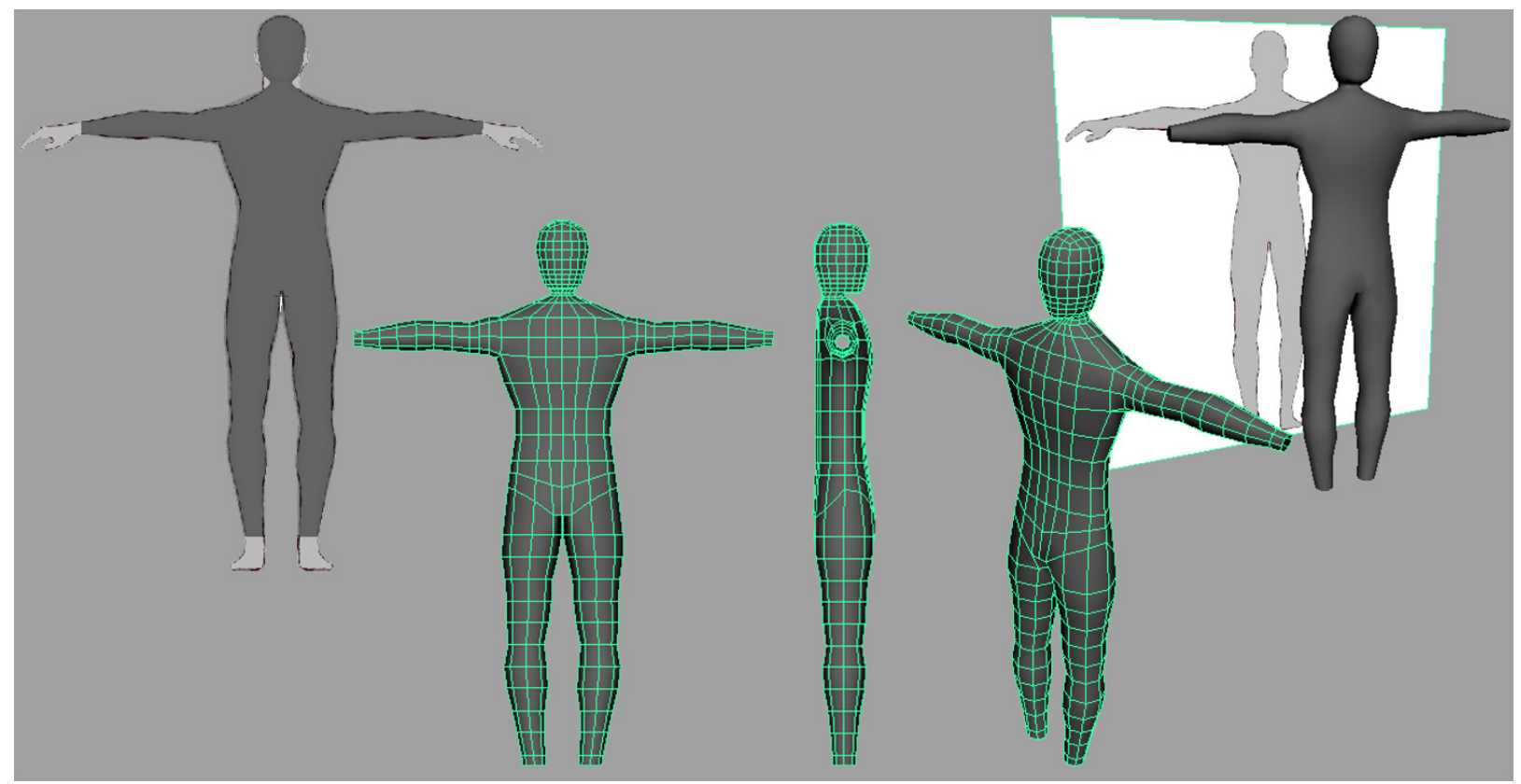

Sketch by Geoffrey Datema (C)

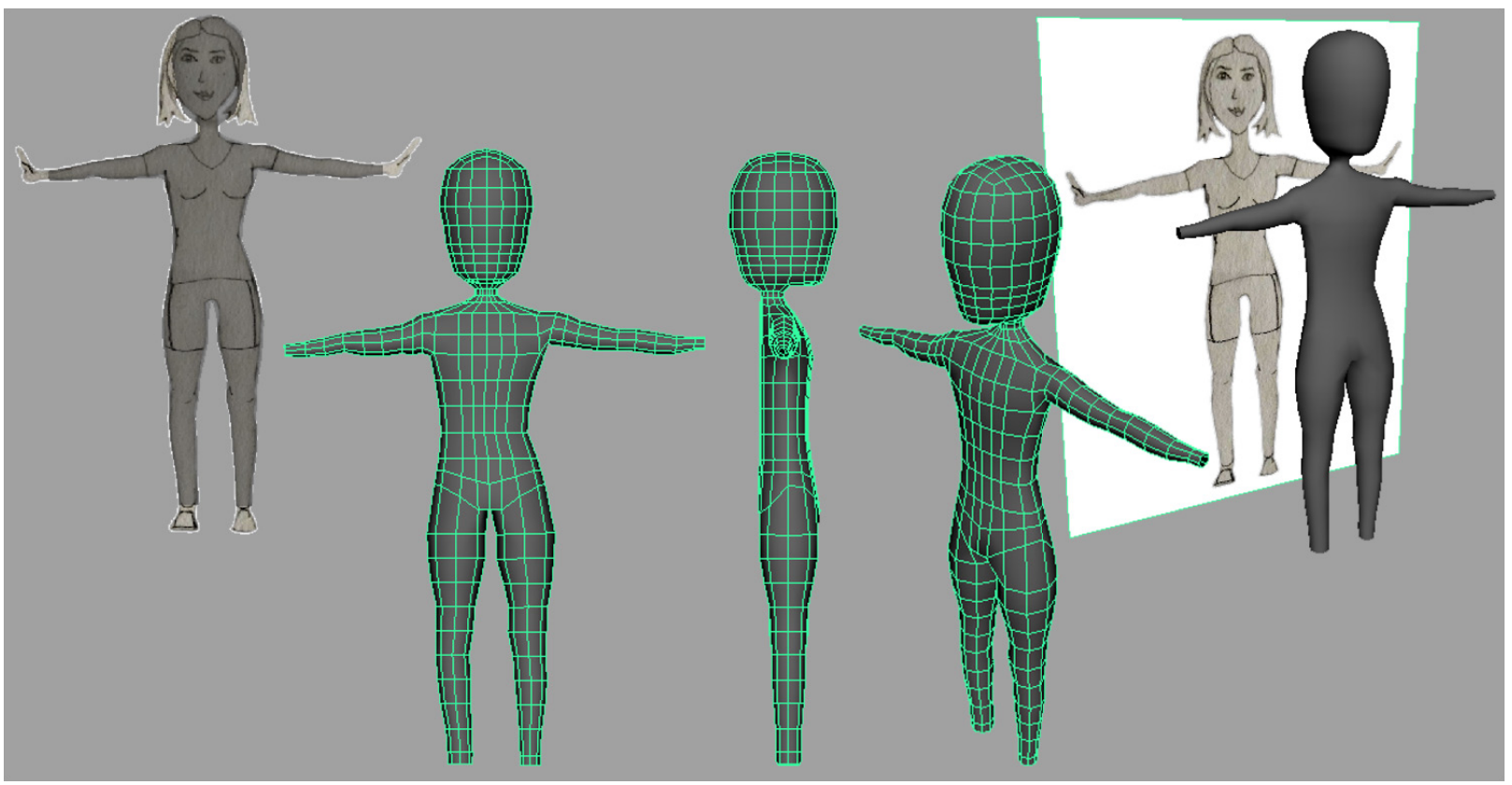

Sketch by Amy Rawding (C) 


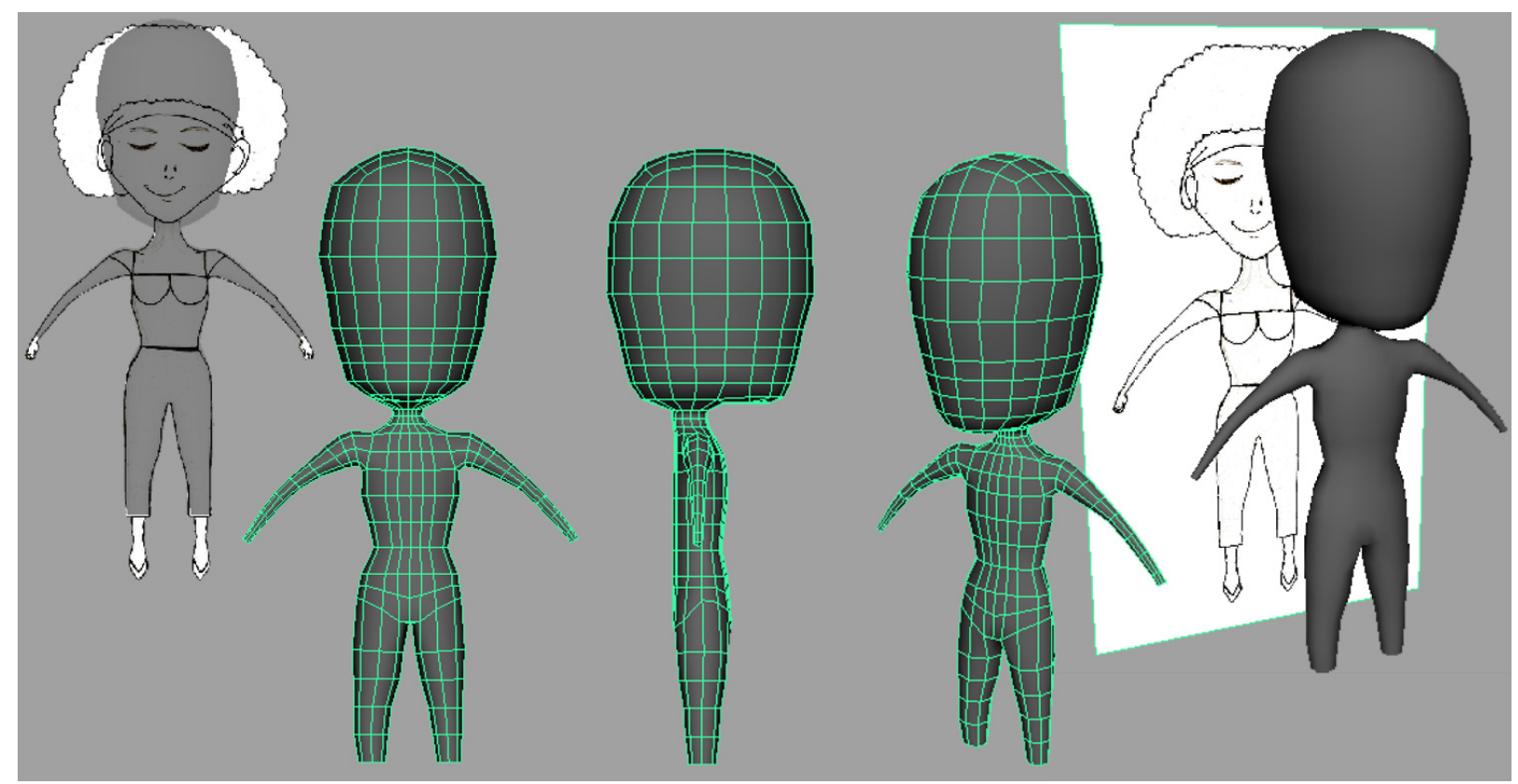

Sketch by Shahad Bakerman (C)

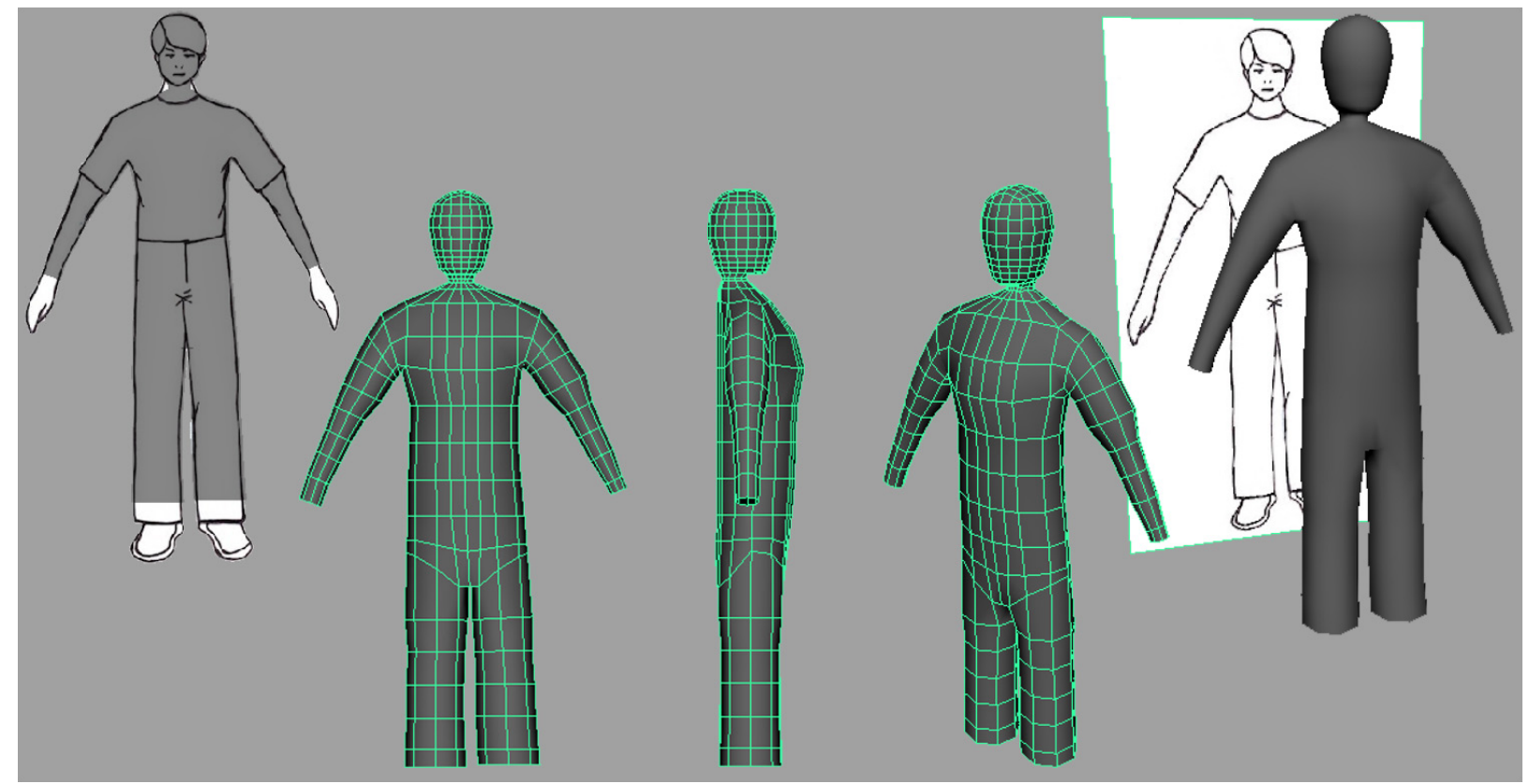

Sketch by Nadeen Rasim (C) 


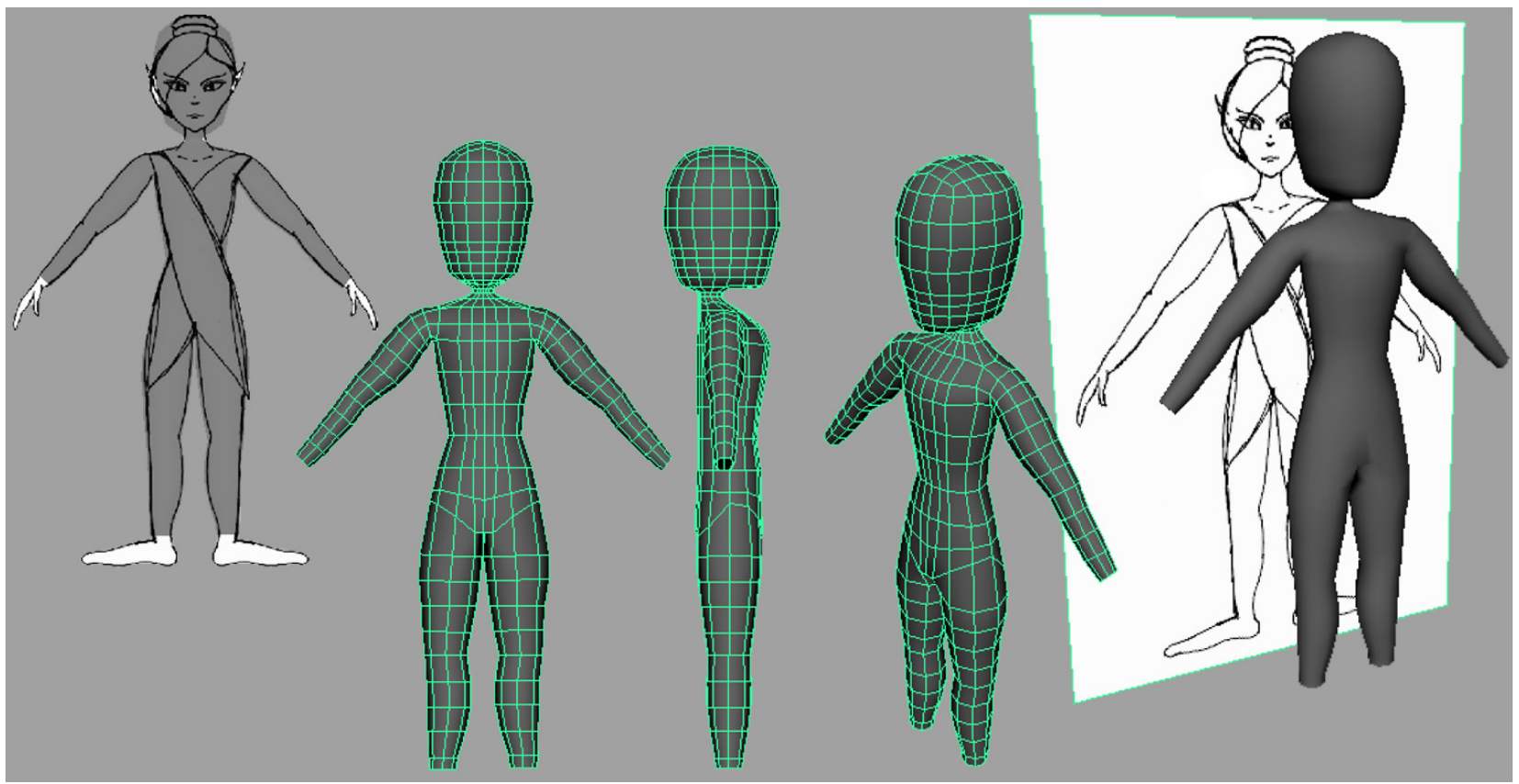

Sketch by Mihaela Petriu (C)

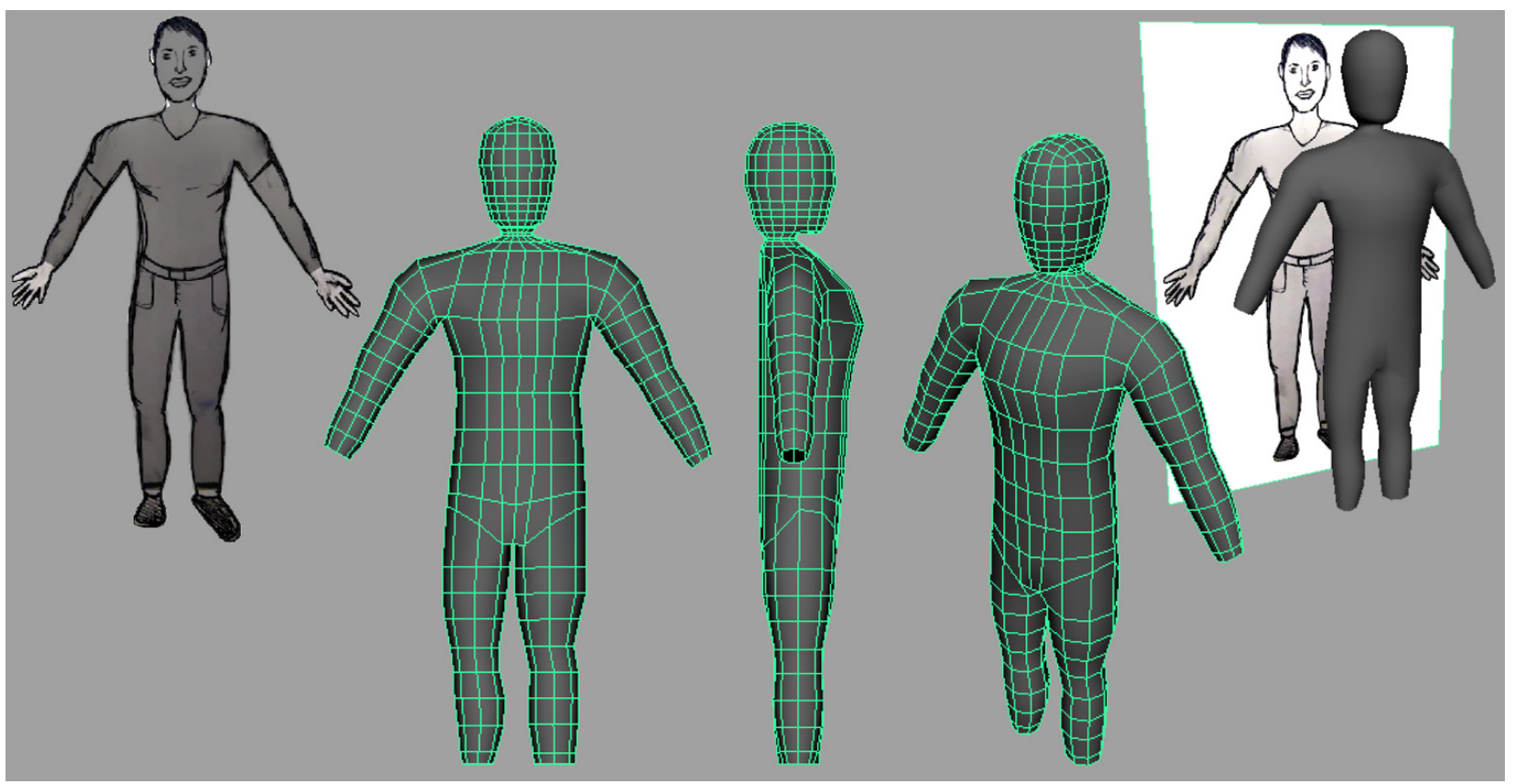

Sketch by Alisa Tkaczyk (C) 


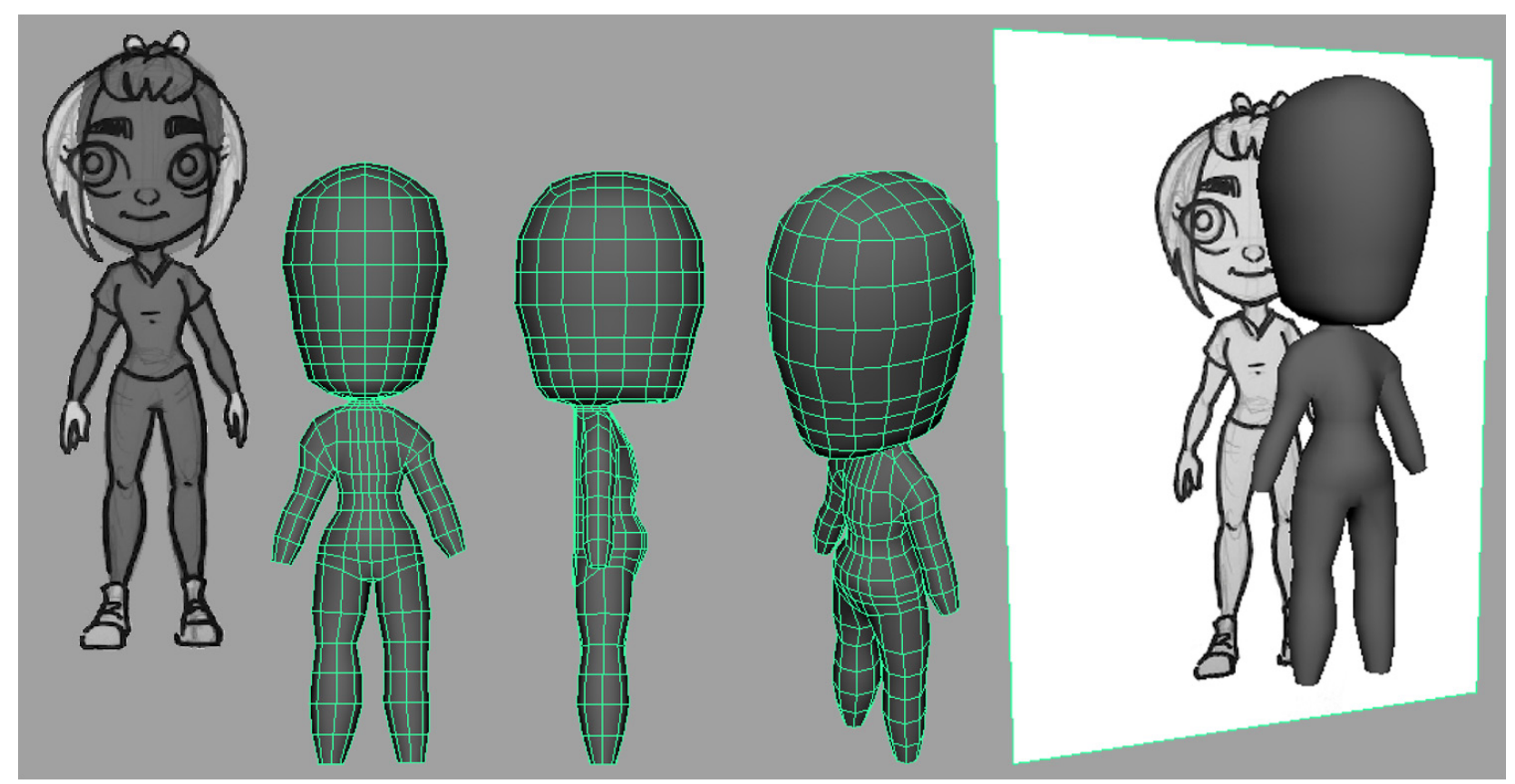

Sketch by Laura Kwan (C)

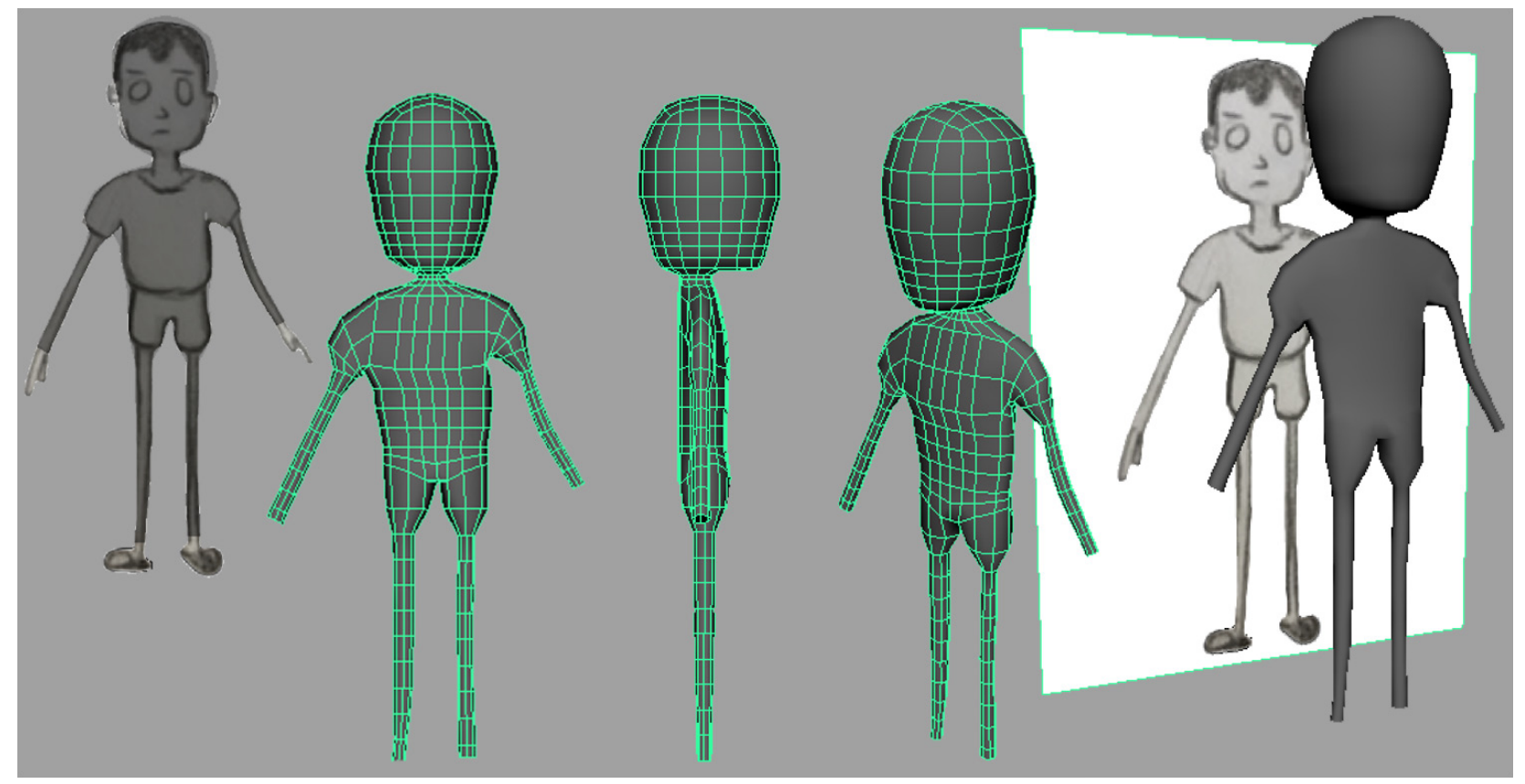

Sketch by Chelsea Liang (C) 


\title{
Appendix C: Consent Form
}

\author{
User Study Consent Form
}

Title: Sketch Characters to 3D Model Block outs

Carleton

U N I V E R S I T Y

Canada's Capital University

Protocol \#: TBD by the REB (as indicated in the clearance form)

Date of ethics clearance: TBD by the REB

Ethics Clearance for the Collection of Data Expires: TBD by the REB

Funders: SSHRC Partnership Grant, IMMERSe Network (http://immersenetwork.com/research-sites/)

I choose to participate in a study

on Sketch Characters to 3D Block outs. This study examines a user interactive computer program that develops a 3D model block out (basic mesh) based on a sketched human character and user input. The researcher for this study is Shaikah Bakerman, a Master's student in the School of Information Technology. She is working under the supervision of Professor Chris Joslin in the School of Information Technology.

This study involves one user study, which will last for approximately 60 minutes, where you will interact with a computer program to produce a 3D block out model based on the sketch image you brought, and then fill an online questionnaire about your experience with the computer program.

With your consent, the researcher will take notes during the study regarding the performance of the interface. Those notes do not consist of any personal comments nor do they link to the participant in any way. Only information and issues regarding the program performance is to be recorded. In addition, the program will record the time duration of each automatic process that the program will perform during the study.

With your consent, your questionnaire answers will be saved and analyzed. To prevent the answers from identifying or linking to you, we kindly ask that you avoid any personal comments when you fill the questionnaire.

You have the right to end your participation anytime during the study by informing the researcher. If you withdraw from the study, all information you have provided will immediately be destroyed.

Because questionnaire answers contain no personal identifiers, it is not possible to withdraw from the user study after you finish the study and leave. The maximum 
time for the participant to withdraw is after the completion of the questionnaire but before leaving the room, where the user study session was taking place.

As a token of appreciation, you will receive a $\$ 15$ Tim Horton's gift card. This is yours to keep, even if you withdraw from the study.

The online questionnaire is created using Google Forms, and all research data, including the questionnaire responses and any notes, will be stored on a Google Drive folder only accessible by the researcher and the research supervisor. All data is resident on Google servers. This data may be subject to the US Patriot Act and could be accessed by US officials. Because the data collected digitally consists of questionnaire responses that have no personal or identifiable links to the user, we will not use any encryption.

At the end of your user study session, your scanned sketch will be securely destroyed from the researcher's computer and your original sketch will be returned to you before you leave the room, and once the project is completed, all research data will be securely destroyed. (Electronic data will be electronically shredded and hard copies will be physically shredded.)

If you would like a copy of the finished research project, you are invited to contact the researcher to request an electronic copy, which will be provided to you.

The ethics protocol for this project was reviewed by the Carleton University Research Ethics Board B, which provided clearance to carry out the research. Should you have questions or concerns related to your involvement in this research, please contact:

CUREB-B contact information:

Dr. Shelley Brown, Chair

Carleton University Research Ethics Board-B

Tel: 613-520-2600 ext. 1505

Shelley.Brown@carleton.ca

\section{Researcher contact information:}

Shaikah Bakerman

Master's Student

School of Information Technology

Carleton University

Email: shaikahbakerman@cmail.carleton.ca
Carleton University Research Office

511 Tory

1125 Colonel By Drive

Ottawa, ON K1S 5B6

ethics@carleton.ca

Signature of participant

Date

Signature of researcher

Date

Supervisor contact information:

Professor Chris Joslin

School of Information Technology

Carleton University

1125 Colonel By Drive

Azrieli Pavilion, Room 230L

Tel: 613-520-2600 ext. 1889

Email: chris joslin@carleton.ca 


\section{Appendix D: Evaluation \& Feedback Questionnaire}

\section{Basic Evaluation}

Rate your experience in 3D modelling (using any 3D software): *

I never did 3D modelling before

Beginner

Intermediate

Advanced

Expert

Rate your experience in 3D human character modelling *

I never created 3D character models

Beginner

Intermediate

Advanced

Expert 


\section{Character Base Mesh}

How long would it take you to manually create the basic geometry of the character sketch you just used? *

A similar level of detail of the mesh that was automatically created

Less than 10 minutes

10-15 minutes

15-30 minutes

30 minutes to an hour

Other:

How would you like to go from a character sketch to a 3D Model? *

To get the basic geometry automatically created for me

To do everything manually

It depends

If you answered (It depends), please comment below:

If it takes the same amount of time, would you prefer to create the basic geometry of the character yourself or automatically? *
Myself
Automatically
It depends

If you answered (It depends), please comment below: 


\section{Software Experience}

Please choose an option in each of the following statements, based on your opinion

The amount of input required from you was: *

Too little Not enough Enough A lot Too much

Why?

The specifications required in the drawn character are: *

Too little Not enough Enough A lot Too much

Why?

Understanding/learning how to use the system was easy: *

$\begin{array}{llll}\begin{array}{l}\text { Strongly } \\ \text { Disagree }\end{array} \text { Disagree } & \text { Neutral } & \text { Agree } & \text { Strongly Agree }\end{array}$

O O $\bigcirc \bigcirc \bigcirc$

Interacting with the software was easy: *

\begin{tabular}{|c|c|c|c|}
\hline $\begin{array}{l}\text { Strongly } \\
\text { Disagree }\end{array}$ & Disagree & Neutral & Agree \\
\hline
\end{tabular}

o 0

Comments about your interaction experience with the software: 


\section{D Mesh Evaluation}

Please choose an option in each of the following statements, based on your opinion

The system generated the 3D mesh quickly: *

$\begin{array}{llll}\text { Strongly } & \text { Disagree } & \text { Neutral } & \text { Agree }\end{array}$

O $\quad 0 \quad 0$

The 3D mesh that was automatically produced by the system is: *

Very Poor Poor $\quad$ Fair Good Gery Good

O

The approximated depth of the mesh is close to how I think the depth of this character should be: *

$\begin{array}{llll}\text { Strongly } & \text { Disagree } & \text { Neutral } & \text { Agree }\end{array}$

O $\bigcirc \bigcirc \bigcirc$


Please rate the following from 1 to 5: 1 (Very Poor) and 5 (Very Good)

\section{Geometry of the mesh: *}

By geometry, we are referring to consistency in polygon type (i.e., quads or 4-sided), and absence of mesh artifacts

\begin{tabular}{llllll}
1 & 2 & 3 & 4 & 5 \\
\hline & 0 & 0 & 0 & 0 \\
\hline
\end{tabular}

Topology and edge flow of the mesh *

By topology and edge flow, we are referring to how smoothly edge loops wrap around the mesh and how evenly spaced those edge loops are

2

3

4

5

$\bigcirc$

○

Geometry and edge flow of the neck: *

Average evaluation of both geometry and edge flow

\begin{tabular}{llllll}
1 & 2 & 3 & 4 & 5 \\
\hline & 0 & 0 & 0 & 0 & 0
\end{tabular}

Geometry and edge flow of the shoulders: *

Average evaluation of both geometry and edge flow

1

2

3

4

5

○

$\bigcirc$

$\bigcirc$

○

$\bigcirc$

Geometry and edge flow of the pelvis/hip: *

Average evaluation of both geometry and edge flow

$\begin{array}{lllll}1 & 2 & 3 & 4 & 5\end{array}$

\begin{tabular}{llllll}
1 & 2 & 3 & 4 & 5 \\
\hline & 0 & 0 & 0 & 0
\end{tabular}

I would use this mesh to build my 3D character: *

Yes

No

Please point the positives and/or negatives you find in the automatically produced mesh *

If applicable, list the main features that you expect in an automatically produced mesh that you would use to build your character 